$$
A \begin{aligned}
& \text { D v a n c E s i n } \\
& \text { gronomy }
\end{aligned}
$$

Volume 120 
AdVANCES IN AGRONOMY 


\section{ADVANCES IN AGRONOMY}

Advisory Board

PAUL M. BERTSCH

University of Kentucky

KATE M. SCOW

University of California, Davis

Emeritus Advisory Board Members

JOHN S. BOYER

University of Delaware

EUGENE J. KAMPRATH

North Carolina State, University

\author{
RONALD L. PHILLIPS \\ University of Minnesota \\ LARRY P. WILDING \\ Texas A\&M University
}

Prepared in cooperation with the

American Society of Agronomy, Crop Science Society of America, and Soil Science Society of America Book and Multimedia Publishing Committee

DAVID D. BALTENSPERGER, CHAIR

LISA K. AL-AMOODI

WARREN A. DICK

HARI B. KRISHNAN

SALLY D. LOGSDON
CRAIG A. ROBERTS

MARY C. SAVIN

APRIL L. ULERY 


\section{ADVANCES IN AGRONOMY}

Edited by

DONALD L. SPARKS

Department of Plant and Soil Sciences

University of Delaware

Newark, Delaware, USA

AMSTERDAM • BOSTON • HEIDELBERG • LONDON NEW YORK • OXFORD • PARIS • SAN DIEGO SAN FRANCISCO $\bullet$ SINGAPORE $\bullet$ SYDNEY • TOKYO 
Academic Press is an imprint of Elsevier

525 B Street, Suite 1900, San Diego, CA 92101-4495, USA

225 Wyman Street, Waltham, MA 02451, USA

32 Jamestown Road, London, NW1 7BY, UK

The Boulevard, Langford Lane, Kidlington, Oxford, OX5 1GB, UK

Radarweg 29, PO Box 211, 1000 AE Amsterdam, The Netherlands

First edition 2013

Copyright (C) 2013 Elsevier Inc. All rights reserved.

No part of this publication may be reproduced, stored in a retrieval system or transmitted in any form or by any means electronic, mechanical, photocopying, recording or otherwise without the prior written permission of the publisher

Permissions may be sought directly from Elsevier's Science \& Technology Rights Department in Oxford, UK: phone (+44) (0) 1865 843830; fax (+44) (0) 1865 853333; email: permissions@elsevier.com. Alternatively you can submit your request online by visiting the Elsevier web site at http://elsevier.com/locate/permissions, and selecting Obtaining permission to use Elsevier material

Notice

No responsibility is assumed by the publisher for any injury and/or damage to persons or property as a matter of products liability, negligence or otherwise, or from any use or operation of any methods, products, instructions or ideas contained in the material herein. Because of rapid advances in the medical sciences, in particular, independent verification of diagnoses and drug dosages should be made

ISBN: 978-0-12-407686-0

ISSN: 0065-2113

For information on all Academic Press publications visit our website at store.elsevier.com

Printed and bound in USA

13141510987654321

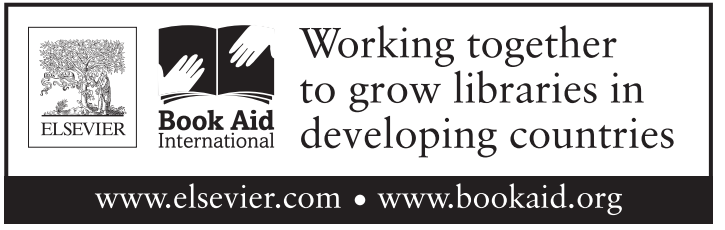




\title{
Food, Nutrition and Agrobiodiversity Under Global Climate Change
}

\author{
Sangam Dwivedi*,1, Kanwar Sahrawat*, Hari Upadhyaya*, \\ Rodomiro Ortiz ${ }^{\dagger}$ \\ *International Crops Research Institute for the Semi-Arid Tropics (ICRISAT), Patancheru, \\ Andhra Pradesh, India \\ tSwedish University of Agricultural Sciences, Department of Plant Breeding and Biotechnology, \\ Sundsvagen, Alnarp, Sweden \\ ${ }^{1}$ Corresponding author: E-mail: sangam375@gmail.com
}

\section{Contents}

1. Introduction 3

2. Moisture Stress and Rising $\mathrm{CO}_{2}$ and Temperature Impacts on Food Quality 6

2.1. Drought, Heat and Grain Quality 7

2.1.1. Protein and Protein Quality 7

2.1.2. Oil and Oil Quality 9

2.1.3. Minerals 9

2.1.4. Carbohydrates 12

2.1.5. Tocopherol (Vitamin E) 14

2.2. Rising $\mathrm{CO}_{2}$, Heat and Grain Quality 14

2.2.1. Protein and Protein Quality 14

2.2.2. Oil and Oil Quality $\quad 15$

$\begin{array}{ll}\text { 2.2.3. Minerals } & 17\end{array}$

2.2.4. Carbohydrates 17

2.3. Elevated $\mathrm{CO}_{2}$ and Forage Quality for Ruminants 17

3. Global Warming and Altered Pathogens and Pests Impacts on
Crop Production and Quality

3.1. Crop Pathogens and Pests in a Changing Climate 21

3.2. Plant Pathogen Scenarios under Climate Change 21

3.3. Emerging Changes in Pest Dynamics under Climate Change 25

3.4. Adapting Crops to Emerging Pathogens and Pests 26

4. Management and Prevention of Aflatoxin 27

4.1. Modeling Climatic Risks to Aflatoxin Contamination 27

4.2. Geostatistics and Geographic Information Systems
to Monitor Spatial Variability in Aflatoxin 30

4.3. High-Throughput and Cost-effective Assays to Detect Aflatoxin 32

4.4. Atoxigenic Fungal Strain as Biocontrol Agent to Manage
Aflatoxin Contamination in Crops

4.5. A System-Based Approach to Control Aflatoxin Contamination 39 
5. Agrobiodiversity to Enhance Nutritional Quality of Food Crops 42

5.1. Global Warming Changes Plant and Soil Biodiversity 42

5.1.1. Plant Biodiversity 42

5.1.2. Soil Biodiversity $\quad 45$

5.2. High-Throughput Assays for Monitoring Nutritional Traits 47

5.2.1. Minerals from the Soil Samples 48

5.2.2. Minerals from Plant Tissues or Grains Samples 49

5.3. Profiling Genetic Variation for Nutritional Traits 52

5.3.1. Variation for Fe, Zn, Phytate and Carotenoids 52

5.3.2. Variation for Protein and Oil Concentrations and Their Quality in Maize 54

5.3.3. Variation for Improving Oil Quality in Peanut 55

5.4. Sustaining Food Quality by Manipulating Soil Microbial Diversity 55

6. Climate Change Analog Locations Representing Future Climate 60

7. Plant Phenomics to Screen Traits for Adapting to Stresses 62

7.1. Root System Architecture 63

7.2. High-Throughput Imaging to Diagnose and Quantify Plant Response 66

7.3. Sensor-Based Phenotyping Platform for Assessing Biomass 69

7.4. Developing Modules to Store, Retrieve, Add or Modify Large Datasets 70

8. Plant Traits to Accelerate Adaptation to Climate Change 71

8.1. Genetic Enhancement for Adaptation to Abiotic Stress 71

8.2. Integrating Trait Diversity to Develop Climate-Proof Nutritious Crops 74

8.2.1. Drought Adaptation in Cereals 74

8.2.2. Submergence and Phosphorus Deficiency Tolerance in Rice 79

8.2.3. Adaptation to Drought in Legumes 81

8.2.4. Salinity Tolerance in Cereals and Legumes 86

8.2.5. Biofortification to Enhancing Nutritional Quality of Food Crops 87

8.3. Genetically Modified Crops Tolerant to Abiotic Stresses 88

9. Outlook 91

Acknowledgments $\quad 95$

$\begin{array}{ll}\text { References } & 95\end{array}$

\section{Abstract}

Available evidence and predictions suggest overall negative effects on agricultural production as a result of climate change, especially when more food is required by a growing population. Information on the effects of global warming on pests and pathogens affecting agricultural crops is limited, though crop-pest models could offer means to predict changes in pest dynamics, and help design sound plant health management practices. Host-plant resistance should continue to receive high priority as global warming may favor emergence of new pest epidemics. There is increased risk, due to climate change, to food and feed contaminated by mycotoxin-producing fungi. Mycotoxin biosynthesis gene-specific microarray is being used to identify foodborn fungi and associated mycotoxins, and investigate the influence of environmental parameters and their interactions for control of mycotoxin in food crops. Some crop wild relatives are threatened plant species and efforts should be made for their in situ conservation to ensure evolution of new variants, which may contribute to addressing 
new challenges to agricultural production. There should be more emphasis on germplasm enhancement to develop intermediate products with specific characteristics to support plant breeding. Abiotic stress response is routinely dissected to component physiological traits. Use of transgene(s) has led to the development of transgenic events, which could provide enhanced adaptation to abiotic stresses that are exacerbated by climate change. Global warming is also associated with declining nutritional quality of food crops. Micronutrient-dense cultivars have been released in selected areas of the developing world, while various nutritionally enhanced lines are in the release pipeline. The high-throughput phenomic platforms are allowing researchers to accurately measure plant growth and development, analyze nutritional traits, and assess response to stresses on large sets of individuals. Analogs for tomorrow's agriculture offer a virtual natural laboratory to innovate and test technological options to develop climate resilience production systems. Increased use of agrobiodiversity is crucial to coping with adverse impacts of global warming on food and feed production and quality. No one solution will suffice to adapt to climate change and its variability. Suits of technological innovations, including climate-resilient crop cultivars, will be needed to feed 9 billion people who will be living in the Earth by the middle of the twenty-first century.

\section{INTRODUCTION}

The world's population will be $\sim 9$ billion in 2050, when the concentration of carbon dioxide $\left(\mathrm{CO}_{2}\right)$ and ozone will be $550 \mathrm{ppm}$ and $60 \mathrm{ppm}$, respectively and the climate will be warmer by $2{ }^{\circ} \mathrm{C}$ (Jaggard et al., 2010). To sufficiently feed these 9 billion people, the total food production will have to be increased by 70\% within 2011-2050 to meet a net demand of $\sim 1$ billion $\mathrm{t}$ of cereals for food and feed and 200 million $\mathrm{t}$ of meat (WSFS, 2009). The evidence accumulated also suggests crop yield decline at temperatures above $30{ }^{\circ} \mathrm{C}$ (Boot et al., 2005; Schlenker and Roberts, 2009). Likewise crop quality will be likely less nutritious, thereby spreading more malnutrition in the developing world (Dwivedi et al., 2012 and the references therein).

Climate models predict that warmer temperatures and increases in the frequency and duration of drought during the twenty-first century will have negative impact on agricultural productivity (Lobell and Field, 2007; Kucharik and Serbin, 2008; Battisti and Naylor, 2009; Schlenker and Lobell, 2010; Roudier et al., 2011; Thornton et al., 2011; Lobell et al., 2011a,b). For example, maize production in Africa could be at risk of significant yield losses as researchers predict that each degree-day that the crop spends above $30{ }^{\circ} \mathrm{C}$ reduces yields by $1 \%$ if the plants receive sufficient water (Lobell et al., 2011a); these predictions are similar to those reported for maize yield 
in the USA (Schlenker and Roberts, 2009). Lobell et al. (2011a) further showed that maize yields in Africa decreased by $1.7 \%$ for each degree-day the crop spent at temperature of over $30{ }^{\circ} \mathrm{C}$ under drought. Wheat production in Russia decreased by almost one-third in 2010, largely due to the summer heat wave (http://www.faostat.fao.org); similarly, wheat production declined significantly in China and India in 2010, largely due to drought (http://www.fao.org/giews/english/alert/index.htm) and sudden rise in temperature respectively, thereby causing forced maturity (Gupta et al., 2010). Warming at $+2{ }^{\circ} \mathrm{C}$ is predicted to reduce yield losses by $50 \%$ in Australia and India (Asseng et al., 2011; Lobell et al., 2012). Likewise, the global maize and wheat production, as a result of warming during the period from 1980 to 2008, declined by $3.8 \%$ and 5.5\%, respectively (Lobell et al., 2011b).

Climatic variation and change are already influencing the distribution and virulence of crop pest and diseases, but the interactions between the crops, pests and pathogens are complex and poorly understood in the context of climate change (Gregory et al., 2009). There is a growing awareness among academicians and policy makers to better appreciate the degree of health risk posed by climate change and formulate strategies that minimize adverse impacts. We need to integrate plant biology into the current paradigm with respect to climate change and humans and animals health to succeed in defeating emerging pests and pathogens posing a new threat to agriculture due to climate change (Patz and Kovats, 2002; McMichael et al., 2006; Ziska et al., 2009).

The evidence to-date suggests that global warming is significantly impacting human and livestock health (McMichael et al., 2006; Patz and Olson, 2006; Jones et al., 2008; Campbell-Lendrum et al., 2009). Mycotoxins of greatest concerns are aflatoxins, deoxynivalenol (DON), fumonisins, and ergot in food crops (Russell et al., 2010; Magan et al., 2011). Climate is a key driving force for fungal colonization and mycotoxin production (Magan et al., 2003) with potential to cause severe economic losses to growers. For example, the annual losses to the US growers from mycotoxin contamination exceed US\$ 1 billion, with maize growers bearing the largest burden (Vardon et al., 2003). Both pre- and postharvest factors contribute to mycotoxin contamination in food and feed crops. The ability of the fungi to produce mycotoxins is largely influenced by temperature, relative humidity, insect attacks and stress conditions of the plants (Miraglia et al., 2009). Worldwide, mycotoxins cause a large number of diseases and human death annually (Lewis et al. 2005; Liu and Wu, 2010; Williams et al., 2004, 2010). 
The largest outbreak of aflatoxicosis has been reported from rural Kenya, resulting in 125 deaths, due to consumption of maize contaminated with mycotoxin (Lewis et al., 2005).

Agrobiodiversity consists of the biological resources that are important for food production, including plants, animals, fisheries, and microorganisms that sustain the functioning of agroecosystems. Climate change poses a serious threat to species fitness (Bell and Collins, 2008; Kelly and Goulden, 2008), and to ecosystem services essential to food production (Shanthi Prabha et al., 2011). The latest database on world plant genetic resources highlighted that there are still large gaps, more specifically in crop wild relatives (CWR) and landraces, in ex situ gene bank collections preserved across the globe (Maxted et al., 2012). There is continuing need to assemble and screen germplasm strategically and discover new sources of variation that will enable developing new crop cultivars adapted to adverse climate and its variability. CWR have contributed many agronomically beneficial traits in shaping the modern cultivars (Dwivedi et al., 2008), and they will continue to provide useful genetic variation for climate-change adaptation, and also enable crop genetic enhancers select plants that will be well-suited for the future environmental conditions (Jarvis et al., 2008a). Promoting onfarm conservation may allow genes to evolve and respond to new environments that would be of great help to capture new genetic variants that will help mitigate climate-change impacts (Rana and Sharma, 2009).

Climate change is imposing significant stresses upon agriculture at a time when more food is required for an increasing world population. To feed $\sim 9$ billion people by the middle of the twenty-first century, the production of high-quality food must increase with reduced inputs. Plant breeding must therefore focus on traits that improve nutritional quality, confer enhanced nutrients- and water-use efficiency (WUE), and those that enhance adaptation to abiotic and biotic stresses to increase yield. New cultivars and breeding populations will need to be continually developed to help withstand climatic extremes and maintain or even increase productivity in the face of increased climatic variability (Ortiz et al., 2008a; Ainsworth and Ort, 2010; Ceccarelli et al., 2010; McClean et al., 2011).

Climate change is altering the availability of resources and the conditions that are crucial to plant performance. Plants respond to these changes through environmentally induced shift in phenotype (phenotypic plasticity). Understanding these responses is crucial to predict and manage the effects of climate change on native species as well as crop plants. The evidence to-date suggests that breeding for phenotypic plasticity in traits other than 
yield will potentially afford resilience in increasingly unpredictable environments (Sambatti and Caylor, 2007; Nicotra and Davidson, 2010; Nicotra et al., 2010). Modern tools such as those from applied genomics or transgenics must support conventional breeding to accelerate development of improved open pollinated or inbred cultivars and hybrids in such a way that it increases the available genetic diversity to improve food and nutritional security (Takeda and Matsuoka, 2008; Tester and Langridge, 2010; Dwivedi et al., 2007a, 2010; Fedoroff et al., 2010; Brummer et al., 2011; McClean et al., 2011; Ronald, 2011). However, genetically enhanced seed-embedded technology should be integrated into ecologically sustainable farming systems and evaluated in the light of their environmental, economic and social impacts in order to develop sustainable agricultural systems (Ronald, 2011).

Researchers are currently engaged with identifying climate analog sites across space (between locations) or time (with past or future climates). Once they are identified, these climate analog sites will provide platforms to develop and test various adaptation strategies including genetically enhanced seed-embedded technology to mitigate the adverse effects of global warming on agricultural productivity (Ramirez-Villegas et al., 2011).

This chapter reviews the role of agrobiodiversity in enhancing food and nutritional security, the contribution of plant phenomics for rapid, accurate and cost-effective assays for identifying traits conferring adaptation to stresses; and assesses the progress made in selected crops toward developing climate-ready crop cultivars adapted to climate change and its variability in the twenty-first century. Issues related to lack of food safety and the outbreaks of new pests due to global warming, and approaches to overcome them are also highlighted.

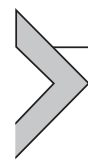

\section{MOISTURE STRESS AND RISING $\mathrm{CO}_{2}$ AND TEMPERATURE IMPACTS ON FOOD QUALITY}

Grain quality (excluding grain physical characteristics) refers to the variation in protein and oil contents, protein and oil quality, carbohydrate, minerals (macro- and micronutrients), and vitamins. These characteristics together determine the quality of food and feed crops. The physical characteristics of the grains include grain size and shape, grain color, and grain weight. Drought and heat invariably reduce grain weight (Prasad et al., 2008; Thomas et al., 2009; Balla et al., 2011), while elevated $\mathrm{CO}_{2}$ increases grain weight (Uprety, 2007; Högy and Fangmeier, 2008). Further, elevated $\mathrm{CO}_{2}$ also causes variation in seed length and width among wheat species; 
being hexaploid wheat more responsive positively to increased seed length and breadth (Uprety et al., 2009).

Extensive literature search revealed that unlike in the case of adverse effects of drought on crops performance (Dwivedi et al., 2010 and references therein), scanty research on the effects of elevated $\mathrm{CO}_{2}$, drought and heat on grain quality.

\subsection{Drought, Heat and Grain Quality}

\subsubsection{Protein and Protein Quality}

The effects of drought or heat on grain quality have been investigated in cereals, pulses and oilseeds (Table 1.1). With few exceptions, drought invariably increased grain protein by $\sim 14-21 \%$ in faba bean, peanut, rice and wheat. The pattern of drought also reflected variation in grain protein. For example, the midseason drought stress in pearl millet raised grain protein by $18 \%$, while the terminal drought stress elevated grain protein by $44 \%$ (Mahalakshmi et al., 1985). The midseason drought in peanut had no adverse effect on grain protein, while terminal drought increased grain protein by 16\% (Dwivedi et al., 1996). Unlike cereals, drought in lupins substantially reduced grain protein by 19-35\%, with greatest reduction observed from lupins grains harvested at 75\% moisture deficit (Khalil and Ismael, 2010).

Drought stress invariably leads to rise in air and soil temperature, which alone or with drought adversely impact the protein content and its quality. For example, at $32 / 26{ }^{\circ} \mathrm{C}$, the protein declined by $19.6 \%$ in groundnut, at $4{ }^{\circ} \mathrm{C}$ increase above the ambient temperature, it declined by $6.7 \%$ in rice, while it continued to increase with rise in temperature, but above $40 / 30{ }^{\circ} \mathrm{C}$, it declined sharply in soybean (Table 1.1). Drought and heat together increased grain protein by $28-34 \%$ in wheat. Protein composition, which is the most decisive factor in bread-making quality, is greatly influenced by various types of stresses. The wheat crop exposed to drought and heat $\left(35^{\circ} \mathrm{C}\right)$ at grain filling significantly altered protein composition: gluten either reduced (Ozturk and Aydin, 2004) or increased (Shahryari et al., 2011), while unextractable polymeric protein fraction and glutenin-to-gliadin ratio was reduced (Balla et al., 2010), with drought alone greatly influencing the protein composition than heat (Balla et al., 2011). Thus, reductions in unextractable polymeric protein fraction and glutenin-to-gliadin ratio indicate a poorer grain quality, despite the higher grain protein under drought in wheat (Balla et al., 2010). Temperature above $35^{\circ} \mathrm{C}$ also led to loss of dough strength in wheat, and the mechanism involved in dough weakening (if known) should provide breeders with selection tools to assist in the production of cultivars that will tolerate heat (Wrigley, 2006). 
Table 1.1 Effect of drought or heat stress on grain protein and protein quality in faba bean, lupins, maize, peanut, pearl millet, rice, soybean, and wheat Summary of stress effect on grain-protein and protein quality

Reference

\section{Drought stress}

\section{Faba bean}

Drought stress increased protein by $13.7 \%$

Al-Suhaibani, 2009

\section{Peanut}

Terminal drought stress increased protein

Dwivedi et al., 1996 by $15.8 \%$

\section{Lupins}

$75 \%$ water deficit reduced protein by $35 \%$ in

Khalil and Ismael, 2010 comparison to control (35\% water deficit)

Drought stress reduced protein by $19.5 \%$

\section{Maize}

Drought stress reduced protein by $3.9 \%$

Pearl millet

Midseason drought increased protein by $18 \%$, while terminal drought 44\%

\section{Rice}

Drought stress increased protein by $20.9 \%$

Drought stress increased protein by $12.7 \%$

Wheat

Protein and gluten under drought stress, respectively, reduced by $3 \%$ and $6 \%$

Drought stress increased protein by $12.8 \%$ continuous drought stress increased protein by $18.1 \%$, while late water stress by $8.3 \%$

Carvalho et al., 2004

Ali et al., 2010

Mahalakshmi et al., 1985

Fofana et al., 2010

Crusciol et al., 2008

Shahryari et al., 2011

Zhao et al., 2009 Ozturk and Aydin, 2004

\section{Heat stress}

\section{Peanut}

Elevated temperature $\left(32 / 26^{\circ} \mathrm{C}\right)$ significantly

Golombek et al., 1995 decreased protein by $19.6 \%$

\section{Rice}

Elevated temperature (ambient $+4{ }^{\circ} \mathrm{C}$ ) significantly decreased protein by $6.7 \%$

\section{Soybean}

Protein increased with rise in temperature but above $40 / 30{ }^{\circ} \mathrm{C}$ it declined sharply

Protein remained stable between 18 and $30^{\circ} \mathrm{C}$

Ziska et al., 1997

but significantly increased at $33{ }^{\circ} \mathrm{C}$; most amino acids remained unchanged except methionine that substantially increased at the warmest temperature

Thomas et al., 2003

Wolf et al., 1982 
Table 1.1 Effect of drought or heat stress on grain protein and protein quality in faba bean, lupins, maize, peanut, pearl millet, rice, soybean, and wheat-cont'd

Summary of stress effect on grain-protein and protein quality Reference

\section{Heat stress}

\section{Wheat}

High temperature and drought stress increased protein by $28.5 \%$

Drought and heat stress increased protein by $34.4 \%$ but protein quality deteriorated

Fernando et al., 2012

Balla et al., 2011

\subsubsection{Oil and Oil Quality}

Drought significantly reduced grain oil, with greatest reductions in lupins $(50-55 \%)$ and maize (40\%). The reduction in oil content was 32\% in rapeseed, and $5-10 \%$ in peanut and sunflower (Table 1.2). In contrast, heat stress in peanut and soybean increased oil by $20 \%$ and $37 \%$, respectively, while oil content was reduced by $23 \%$ in heat-stressed kidney bean (Table 1.2).

The nutritional and storage quality depend on the relative proportion of saturated and unsaturated (oleic, linoleic and linolenic) fatty acids in the oil. A high proportion of polyunsaturated fatty acid is desirable as it lowers plasma cholesterol and low-density lipoprotein, which may reduce the risk of coronary heart disease and atherogenesis (Jackson et al., 1978). Further, linoleic and linolenic fatty acids have been associated with oxidation and the development of unfavorable flavors (Dutton et al., 1951; Branch et al., 1990). Drought and heat stress, either independently or together, had significant effects on fatty acid composition in maize, peanut, soybean, and sunflower. An increase in oleic acid in general led to a corresponding decrease in linoleic or linolenic fatty acids (Table 1.2). However, differential response of the test materials to drought and heat (Rennie and Tanner, 1989; Ali et al., 2009, 2010) may provide opportunity to identify genotypes with least adverse effect on oil quality.

\subsubsection{Minerals}

Globally, over 3 billion people are affected by micronutrient malnutrition (http://www.unscn.org). Malnourishment is often associated with serious physical incapacity, mental impairment, decreased health and parasitic diseases. The micronutrients are also essential for growth and development of crop plants (Dwivedi et al., 2012 and references cited therein). Few studies in barley, lupin, maize, rice and wheat reported the adverse effect of drought and heat on grain minerals (Table 1.3). Drought stress mostly increased zinc (Zn) in barley, lupin, 
Table 1.2 Effect of drought or heat stress on grain oil and oil quality in kidney bean, lupins, maize, peanut, rapeseed, soybean, and sunflower

Summary of stress effect on grain

oil and fatty acid composition

Reference

\section{Drought stress}

\section{Peanut}

Oil reduced by $5 \%$; oleic acid increased

Dwivedi et al., 1996

by $9.3 \%$ while linoleic acid reduced

by $11.5 \%$

\section{Lupins}

Oil reduced by $50 \%$

Carvalho et al., 2005

Oil reduced by $55 \%$

Carvalho et al., 2004

\section{Maize}

Oil decreased up to $40 \%$; oleic acid increased

Ali et al., 2010

up to $25.6 \%$; linoleic acid reduced up to

$14 \%$; individual $(\alpha, \gamma, \beta)$ and total tocopherol increased substantially

\section{Rapeseed}

Oil reduced by $31.7 \%$

Ahmadi and Bahrani, 2009

\section{Soybean}

Oleic acid increased by $6.5 \%$, while linoleic acid reduced by $3.6 \%$

Kirnak et al., 2010

\section{Sunflower}

Oil decreased by $10.52 \%$; differential response

Ali et al., 2009 due to water stress in changes in oleic, linoleic and linolenic fatty acids between cultivars; $\alpha-, \delta$ - and $\gamma$-tocopherol as well total tocopherol increased by several folds $67-251 \%$

\section{Heat stress}

\section{Peanut}

Oil content increased by $20 \%$ as the temperature

Golombek et al., 1995 increased; oleic $(\mathrm{O})$ acid increased by $24 \%$ with corresponding decrease in linoleic (L) acid and increase in $\mathrm{O} / \mathrm{L}$ ratio, a measure of shelf-life of the product

Elevated temperature significantly increased oleic and stearic acids by $5 \%$ and $9 \%$, respectively, while palmitic and linoleic acids decreased by $3 \%$ and $6 \%$

\section{Kidney bean}

Elevated temperature $\left(34 / 24^{\circ} \mathrm{C}\right)$ significantly decreased oil by $22.7 \%$

Burkey et al., 2007

Thomas et al., 2009 
Table 1.2 Effect of drought or heat stress on grain oil and oil quality in kidney bean, lupins, maize, peanut, rapeseed, soybean, and sunflower-cont'd

\section{Soybean}

Elevated temperature $\left(40 / 30^{\circ} \mathrm{C}\right)$ substantially $\quad$ Rennie and Tanner, 1989 increased oleic acid, while linoleic and linolenic acids correspondingly decreased; however, genotypic differences in response to elevated temperature were noticed

Oil increased by $37 \%$ as temperature increased; Wolf et al., 1982 oleic acid increased by $196 \%$ with corresponding decrease in linoleic and linolenic acids

Table 1.3 Effect of drought or heat stress on grain macro- and micronutrients in barley, lupins, maize, rice, and wheat

Summary of stress effect on grain minerals

Reference

\section{Drought stress}

\section{Barley}

N, Zn, Mn increased by 12, 27 and 7\%

Farahani et al., 2011

\section{Lupins}

Drought stress significantly increased both

Carvalho, 2005 macronutrients ( $\mathrm{Ca}, \mathrm{Na}, \mathrm{K}, \mathrm{Mg}$ ) and micronutrients ( $\mathrm{Fe}, \mathrm{Zn}, \mathrm{Mn}, \mathrm{Cu}$ ) as well as phytate content

\section{Maize}

Drought combined with soil acidity led to Rastija et al., 2010 more than twice the accumulation of $\mathrm{Zn}$ and 6-9 times accumulation of $\mathrm{Mn}$

\section{Rice}

Rainfed rice provided increased grain N (12.7\%), Crusciol et al., 2008 $\mathrm{P}$ (45.4\%), Ca (37.1\%), Mg (152.6\%), Fe (356.6\%) and $\mathrm{Zn}(73 \%)$ compared to sprinklerirrigated grown rice; however, $\mathrm{K}$ reduced by $12.3 \%, \mathrm{~S}$ by $23.1 \%$ and $\mathrm{Cu}$ by $50 \%$

\section{Wheat}

$\mathrm{P}$ increased by $11 \%$, Ca 25\%, Mg 8.3\% and Zn $20.8 \%$; however, K reduced by $10.5 \%$

Zhao et al., 2009

\section{Heat stress}

\section{Wheat}

Elevated temperature increased Fe by $25 \%$, Zn $24.5 \%$, S 23\%, and Ca 6\%

$\mathrm{N}$ and $\mathrm{P}$ continued to increase with temperature up to $40 / 30{ }^{\circ} \mathrm{C}$, then declined

Fernando et al., 2012

Thomas et al., 2003 
maize, rice and wheat; iron $(\mathrm{Fe})$ in lupin and rice; calcium $(\mathrm{Ca})$ in lupin, rice and wheat; phosphorus $(\mathrm{P})$ in rice and wheat; nitrogen $(\mathrm{N})$ in barley and rice; potassium $(\mathrm{K})$ in lupin (but reduced in rice and wheat); magnesium $(\mathrm{Mg})$ in lupin, rice and wheat; and manganese $(\mathrm{Mn})$ in barley and maize. Sodium $(\mathrm{Na})$ in lupin increased, while sulfur $(\mathrm{S})$ and copper $(\mathrm{Cu})$ declined in rice. However, drought brings variable changes in macro- and micronutrients in these crops. For example, produce from rainfed rice showed almost four and half times more grain Fe $\left(51.6 \mathrm{mg} \mathrm{kg}^{-1}\right)$ than those recorded from produce harvested from sprinklergrown rice crop (11.3 $\mathrm{mg} \mathrm{kg}^{-1}$ ) (Crusciol et al., 2008). Likewise, drought combined with soil acidity led to the excessive accumulation (6-9 times) of Mn in maize grains (Rastija et al., 2010), while maize grains obtained from acidic soils showed much higher $\mathrm{Mn}$ and $\mathrm{Zn}$ than those from nonacid soils (Rastija et al., 2010). Variation in soil water also impacted grain minerals (P, K, Ca, Mg and $\mathrm{Zn}$ ), with highest increase of $20.8 \%$ detected for $\mathrm{Zn}$ in plots receiving $45 \%$ of soil water than those that received $85 \%$ soil water $\left(45.2 \mathrm{mg} \mathrm{kg}^{-1} \mathrm{Zn}\right)$ in winter wheat (Zhao et al., 2009). In sweet corn, $30 \%$ water deficit in comparison to no water deficit reduced grain Fe by $57 \%$, Zn by $43 \%$ and Cu by $47 \%$ (Oktem, 2008). Recent research has shown that postflowering drought stress, in comparison to no stress, significantly increased grain Fe (24-35\%) and Zn (15-20\%) concentrations in pearl millet (ICRISAT, unpublished data). Minerals in grains were differently affected by drought stress (Peleg et al., 2008), providing an opportunity to select germplasm with least difference between stressed and nonstressed conditions. Wheat grains harvested from heat-stressed plots showed 23-25\% greater Fe, Zn and S. However, Ca increased by 6\% (Fernando et al., 2012), while in another study, $\mathrm{N}$ and $\mathrm{P}$ continued to increase with increasing temperature up to $40 / 30^{\circ} \mathrm{C}$, and then declined (Thomas et al., 2003).

\subsubsection{Carbohydrates}

Carbohydrates are one of the main dietary components. They are sugars, starches and fibers, classified either as monosaccharide (glucose and fructose), disaccharide (table sugar) or complex (starches) carbohydrates. All of them provide energy to the human body. Up-to-date literature search reveals that lupin and faba bean among the legumes and maize and wheat among the cereals have been investigated for the effects of drought stress on their grain carbohydrate (Table 1.4). Lupin grains harvested from crops with $75 \%$ water deficit showed $30 \%$ reduction in carbohydrate in comparison to those obtained from the crop that suffered 35\% water deficit (Khalil and Ismael, 2010); while earlier reports revealed varying effects of drought stress on grain carbohydrate in lupin (Carvalho et al., 2004, 2005). Drought stress in faba bean caused only marginal increase $(4.3 \%)$ in carbohydrate, while it increased fiber by $7-21 \%$ and sugar by $33-35 \%$ in maize (Ali et al., 2010). 
Table 1.4 Effect of drought or heat stress on grain carbohydrate in faba bean, lupins, maize, peanut, soybean and wheat

Summary of stress effect on grain carbohydrate, fiber and starch contents

Reference

\section{Drought stress}

\section{Faba bean}

Carbohydrate increased by $4.3 \%$

Al-Suhaibani, 2009

\section{Lupins}

Water deficit (75\%) caused 30\% reduction in

Khalil and Ismael, 2010 carbohydrate in comparison to $35 \%$ water deficit

Total carbohydrate, sucrose and sucrose/galactoside ratio increased; however, raffinose reduced

Soluble sugars decreased by $18.25 \%$, crude fiber by $10.6 \%$ and starch by $42.6 \%$

\section{Maize}

Fiber increased by $7.3-21.3 \%$, starch by $9.1-9.2 \%$; sugar by $33-35 \%$

\section{Wheat}

Starch reduced by $20.5 \%$

Zhang et al., 2010

Starch reduced by $3.4 \%$

Carvalho et al., 2005

Carvalho et al., 2004

Ali et al., 2010

\section{Heat stress}

\section{Peanut}

Elevated temperature $\left(32 / 26^{\circ} \mathrm{C}\right)$ significantly

Golombek et al., 1995 decreased total sugar by $24.5 \%$ and starch by $53 \%$

\section{Soybean}

$\mathrm{CO}_{2}$-induced elevated temperature decreased total

Thomas et al., 2003 nonstructural carbohydrate (TNC), with more reduction in soluble sugars than the starch

Glucose, fructose and raffinose remained unaffected while sucrose declined by $56 \%$ at $33 / 28{ }^{\circ} \mathrm{C}$

Wolf et al., 1982

Starch constitutes the major component of the grains, which serves as a multifunctional ingredient for the food industry. The shape, volume and structure determine the starch quality. Drought stress in wheat reduced grain-starch by up to 20\% (Zhao et al., 2009; Zhang et al., 2010), while it increased grain-starch by $9 \%$ in maize (Ali et al., 2010). Drought also brought changes in the proportion of starch granules: A-type granules increased, while B- and C-type granules decreased, and these effects were cultivar- and stage-dependent in wheat (Singh et al., 2008a; Dai et al., 2009).

Elevated temperature $\left(32 / 26^{\circ} \mathrm{C}\right)$ substantially decreased total sugar and starch in groundnut, while glucose, fructose, and raffinose remained unaffected in soybean, but sucrose at $33 / 28{ }^{\circ} \mathrm{C}$ temperature regime declined by $56 \%$ (Table 1.4). Further, $\mathrm{CO}_{2}$-induced elevated temperature in soybean increased 
total nonstructural carbohydrate, with more reduction in soluble sugars than in starch (Thomas et al., 2003). High temperature from anthesis to maturity reduced the duration of starch accumulation in wheat. Starch accumulation ceased approximately 6 days earlier for grain produced under a $37 / 17^{\circ} \mathrm{C}$ and 21 days earlier under a $37 / 28^{\circ} \mathrm{C}$ than for grain produced under a $24 / 17^{\circ} \mathrm{C}$. In comparison to $24 / 17^{\circ} \mathrm{C}$, starch content was approximately $19 \%$ lower for mature grain produced under $37 / 17^{\circ} \mathrm{C}$ and $58 \%$ less under $37 / 28^{\circ} \mathrm{C}$. The smaller B-type granules were the predominant class in mature grain produced under $24 / 17$ and $37 / 17{ }^{\circ} \mathrm{C}$, whereas the larger A-type granules were predominant in grain produced under $37 / 28^{\circ} \mathrm{C}$ (Hurkman et al., 2003).

More recently, Wang et al. (2012) investigated the role of preanthesis high-temperature acclimation in alleviating the negative effects of postanthesis heat stress on stem-stored carbohydrate remobilization and grainstarch accumulation in wheat. Postanthesis heat stress lowered grain-starch content and increased percentages of volume, number and surface area of B-type starch granules in heat at postanthesis as well heat at pre- and postanthesis than in no heat stress situation. However, plants exposed to heat at both stages of development (pre- and postanthesis) had much higher starch content, and caused less modified B-type starch granule size than the plants exposed to high temperature at postanthesis stage, demonstrating that the preanthesis high-temperature acclimation effectively enhanced carbohydrate remobilization from stem to grains, and led to less changed starch content and starch granule size distribution in grains of wheat under postanthesis heat stress.

\subsubsection{Tocopherol (Vitamin E)}

Tocopherols are well recognized as antioxidants in vegetable oils, and their presence increases the stability of lipids against autoxidation (Goffman and Böhme, 2001). Drought in maize and sunflower increased the individual $(\alpha, \beta, \delta)$ as well as total tocopherol by several folds (67-251\%) (Ali et al., $2009,2010)$, whereas similar stress in soybean caused two- to threefold increases in $\alpha$-tocopherol (Steven and Diane, 2002).

\subsection{Rising $\mathrm{CO}_{2}$, Heat and Grain Quality}

\subsubsection{Protein and Protein Quality}

Grain-protein concentration and composition are major determinants of grain nutritional value as well of flour functional properties (Weegels et al., 1996; Feil, 1997; Shewry and Halford, 2002). Wheat flour protein consists of albumins and globulins ( 20\%) and glutens proteins ( 80\%). Albumin and 
globulin are metabolic proteins while gluten, as storage proteins, influences the baking properties. The gluten proteins based on solubility in aqueous alcohol are further divided into soluble gliadins and insoluble glutenins, with both fractions consisting of numerous, partially closely related protein components (Wieser, 2007). Wheat among the cereals is the most extensively studied crop for the effects of elevated $\mathrm{CO}_{2}$ on grain-protein and protein quality (Table 1.5). Elevated $\mathrm{CO}_{2}$ reduced grain protein by $4-15 \%$. Application of varying doses of $\mathrm{N}$ fertilizer under elevated $\mathrm{CO}_{2}$ did not ameliorate the decline in grain protein; however, researchers noted less reduction $(14 \%)$ at $100 \mathrm{~N}$ (Wieser et al., 2008) than when $\mathrm{N}$ was either not applied or applied at low rate (up to 27\% reduction) (Conroy et al., 1994; Porteaus et al., 2009), suggesting thereby that higher $\mathrm{N}$ under elevated $\mathrm{CO}_{2}$ will have some positive effect, but not enough to arrest the decline of grain protein. Furthermore, elevated $\mathrm{CO}_{2}$ also brought significant changes in wheat grain-protein composition: gliadins reduced up to $20 \%$, glutenins up to $15 \%$, and glutenin macropolymer up to $19 \%$, while albumins and globulins fractions were not affected. Within gliadins, $w 5$-gliadins and $w 1,2$-gliadins were more affected than $\alpha$-gliadins and $\gamma$-gliadins, while within glutenins, high molecular weight (HMW) subunits were more affected than low molecular weight (LMW) subunits, thus, adversely impacting baking quality (Wieser et al., 2008).

Grain-protein quality is also influenced by variation in amino acids composition, including essential amino acids (leucine, isoleucine, valine, lysine, threonine, tryptophan, methionine, phenylalanine, and histidine), which are not produced by the body, but must be supplied by food. A comprehensive study in rice showed substantial reduction in amino acids under elevated $\mathrm{CO}_{2}$ conditions, with essential amino acids reduced between 29\% and 38\% (Xu et al., 1998). Methionine was substantially increased in soybean at the warmest temperature, while the other amino acids remained unchanged (Wolf et al., 1982). More recently, Högy and Fangmeier (2008) detected $8-22 \%$ reduction in amino acid composition depending on the exposure system and rooting volume in wheat.

\subsubsection{Oil and Oil Quality}

Elevated $\mathrm{CO}_{2}$ is associated with increased global warming. Essentially, high temperature reduced the oil content per se but improved oil quality (as determined by variation in fatty acid composition): oleic acid increased, while linoleic or linolenic acids linearly decreased in oil crops (see Section 2.1).

Omega fatty acids (omega-3 and omega-6), which are not synthesized in the body but obtained through food source or as supplement, are 
Table 1.5 Effect of elevated carbon dioxide $\left(\mathrm{CO}_{2}\right)$ on grain protein and protein quality in barley, rice, and wheat

Summary of rising $\mathrm{CO}_{2}$ effect on grain-protein and protein quality

Reference

\section{Barley}

Protein reduced by $11-13 \%$

Rice

Protein reduced by $9 \%$

Total amino acids at elevated $\mathrm{CO}_{2}$ were lowered by $30 \%$; except for cystine (increased by $11 \%$ ) and arginine (increased by $21.7 \%$ ), all other 15 amino acids of rice grains were $28-40 \%$ lower under elevated $\mathrm{CO}_{2}$, including essential amino acids, lysine, threonine, methionine, phenylalanine, leucine, and isoleucine

\section{Wheat}

Protein reduced by $12.7 \%$

Protein reduced by $4-13 \%$

Fernando et al., 2012

Erbs et al., 2010

Protein reduced by $3.5 \%$

Protein reduced by $26.8 \%$ at elevated $\mathrm{CO}_{2}$ and low N supply

Protein reduced by $7.4 \%$, changes in amino acid composition with greater reduction in nonessential than essential amino acids

Amino acids such as Thr, Val, Ile, Leu, Arg, Tyr, Asp, Ser, Gln, Ala and Phe reduced significantly by 7.7-22.2\% depending on exposure system and rooting volume

Protein reduced by $9 \%$ at N50 and $14 \%$ at N100; substantial effects on protein fractions-gliadins reduced by $13-20 \%$, glutenins by $15 \%$, glutenin macropolymer by 16-19\%; diminishing baking quality

Protein in grain reduced by $6.25 \%$, while in flour by $12.5 \%$

Protein reduced by $15.2 \%$ and lysine by $5.8 \%$

Protein reduced by $13.9 \%$

Högy et al., 2009a

Porteaus et al., 2009

Högy et al., 2009b

Högy and Fangmeier, 2008

Wieser et al., 2008

Ziska et al., 2004

Wu et al., 2004

Bluementhal et al., 1996

Protein reduced by 9-14\%, highest reduction under

Conroy et al., 1994 zero $\mathrm{N}$ in comparison to limited $\mathrm{N}$ application

associated with a range of beneficial health effects in humans (Covington, 2004). Fish is a good source of omega-3 fatty acids. However, there is a growing concern about the presence of organic contaminants in seafood (Hites et al., 2004). Hence, we need to find alternative sources of omega-3 
fatty acids. Ziska et al. (2007) were probably the first to demonstrate the effects of enriched $\mathrm{CO}_{2}$ on omega fatty acids in mungbean-omega- 6 fatty acids reduced, while omega-3 fatty acids significantly increased in mature grains-which demonstrate that mungbean produced under elevated $\mathrm{CO}_{2}$ could be an alternative source of omega- 3 fatty acids in the diet.

\subsubsection{Minerals}

Limited studies in barley, rice \& wheat have shown that rising $\mathrm{CO}_{2}$ has major impact on cereal grain micronutrients (Table 1.6). For example, grain $\mathrm{Fe}$ and $\mathrm{Zn}$ were significantly reduced under elevated $\mathrm{CO}_{2}$ conditions in rice and wheat. The reductions in Fe ranged between 10\% and 29\%, while the reduction in $\mathrm{Zn}$ varied from $17 \%$ to $33 \%$. Some minerals responded differently: $\mathrm{K}$ and $\mathrm{Ca}$ increased by $12-41 \%$ in rice, but decreased from $12 \%$ to $23 \%$ in wheat. The decline in grain $\mathrm{N}$ was in the range of $12-22 \%$ in rice and $15-29 \%$ in wheat. Soil $\mathrm{N}$ also impacted grain minerals. For example, elevated $\mathrm{CO}_{2}$ and low soil $\mathrm{N}$ decreased $\mathrm{S}$ by $14 \%$ in barley grains, while it increased by $5 \%$ in wheat grains. Statistically nonsignificant changes were also noted with respect to other macro- and micronutrients. It is therefore clear that produce harvested from elevated $\mathrm{CO}_{2}$ conditions will have altered grain mineral contents.

\subsubsection{Carbohydrates}

Elevated $\mathrm{CO}_{2}$ also brought changes in grain carbohydrate in rice and wheat (Table 1.7). For example, total sugars and nonstructural carbohydrates substantially increased in rice grains (Uprety et al., 2007). Variation in soil N and enriched $\mathrm{CO}_{2}$ adversely impacted hemicellulose in wheat-at low $\mathrm{N}$ and high $\mathrm{CO}_{2}$, the hemicellulose reduced by $26 \%$, while at high $\mathrm{N}$ and enriched $\mathrm{CO}_{2}$, the decline in hemicellulose was only $13 \%$. Furthermore, starch content increased by $7-8 \%$ under elevated $\mathrm{CO}_{2}$ irrespective of the variation in soil $\mathrm{N}$, while water-soluble carbohydrates reduced by $7-15 \%$ at low/high $\mathrm{N}$ supply under elevated $\mathrm{CO}_{2}$ in wheat (Porteaus et al., 2009). Elevated $\mathrm{CO}_{2}$ or high temperature also impacted grain carbohydrate in kidney bean: glucose was reduced, while sucrose and raffinose were increased (Thomas et al., 2009).

\subsection{Elevated $\mathrm{CO}_{2}$ and Forage Quality for Ruminants}

Ruminants (cattle, sheep or goat) have evolved a four-compartment capacious pregastric stomach where a symbiotic relationship exists with microbes that have an ability to break down complex structural polysaccharides to 
Table 1.6 Effect of elevated carbon dioxide $\left(\mathrm{CO}_{2}\right)$ on grain macro- and micronutrients in barley, rice, and wheat

Summary of rising $\mathrm{CO}_{2}$ effect on grain macroand micronutrients

Reference

\section{Barley}

$\mathrm{S}$ reduced on average by $14 \%$ under elevated

Erbs et al., 2010

$\mathrm{CO}_{2}$ and low $\mathrm{N}$ supply

\section{Rice}

Ca increased by $12.5 \%$ and $\mathrm{K}$ by $41.2 \%$, while

$\mathrm{N}$ decreased by $2.5 \%$

$\mathrm{P}$ declined by $5 \%$, Zn $28 \%$ and Fe 17\%;

$\mathrm{N}$ reduced in the range of $12-22 \%$

\section{Wheat}

Fe reduced by $10.5 \%$, Zn $17 \%$, S $7.5 \%$, and Ca $12 \%$

$\mathrm{S}$ reduced on average by $5 \%$ under elevated $\mathrm{CO}_{2}$ and low $\mathrm{N}$ supply

$\mathrm{Na}, \mathrm{Ca}, \mathrm{P}, \mathrm{S}, \mathrm{Fe}, \mathrm{Zn}, \mathrm{Cu}, \mathrm{Mn}$ and $\mathrm{Al}$ decreased, while $\mathrm{K}, \mathrm{Mg}$ and Mo increased; however, changes were statistically nonsignificant

$\mathrm{K}, \mathrm{Mo}, \mathrm{Pb}$ significantly increased, while $\mathrm{Mn}, \mathrm{Fe}$, $\mathrm{Cd}$ and Si significantly decreased

$\mathrm{Na}, \mathrm{Ca}, \mathrm{Mg}, \mathrm{S}, \mathrm{Fe}, \mathrm{Zn}$ and $\mathrm{Mn}$ decreased by $3.7-18.3 \%$

$\mathrm{N}$ decreased by $15.2 \%, \mathrm{P} 36.6 \%, \mathrm{~K} 23.2 \%$ and Zn 32.6\%

N, S, Fe and Zn reduced between 21 and 29\%, Ca $12-17 \%, \operatorname{Mg} 8-13 \%$ and Mn 6-8\%

Uprety, 2007

Seneweera and Conroy, 1997

Fernando et al., 2012

Erbs et al., 2010

Högy et al., 2009a

Högy et al., 2009b

Högy and Fangmeier, 2008

Wu et al., 2004

Manderscheid et al., 1995

compounds easily absorbed by the animal. Ruminant digestion is complex as a result of interactions among the diet, the microbial population, and the animal (Owensby et al., 1996; Ehleringer et al., 2002). The plants in $\mathrm{CO}_{2-}$ enriched environments grow faster, produce more biomass and grain yield (Jaggard et al., 2010). However, this rapid growth often leads to poor nutritional quality of the forage (Akin et al., 1995; Cotrufo et al., 1998; Sinclair et al., 2000; Lilley et al., 2001; Pal et al., 2004; Pang et al., 2005). The nutritive value of the forage is highly dependent on leaf $\mathrm{N}$, protein, fiber, nonstructural carbohydrates and minerals. The reduced $\mathrm{N}$ and other elements and increased fiber concentrations in plants grown under elevated $\mathrm{CO}_{2}$ may adversely impact ruminant productivity, unless ruminants are supplemented with additional nutrition in their diets. Besides changes in leaf chemistry, reduction in forage quality may also come from morphological changes associated with 
Table 1.7 Effect of rising carbon dioxide $\left(\mathrm{CO}_{2}\right)$ and elevated temperature on grain carbohydrate in kidney bean, rice, and wheat

Summary of rising $\mathrm{CO}_{2}$ effect on grain carbohydrate, fiber and starch

\section{Reference}

\section{Elevated $\mathrm{CO}_{2}$}

\section{Rice}

Total sugar increased by $32.5 \%$, total nonstructural carbohydrate by $29.3 \%$ and amylase by $5.2 \%$

Uprety, 2007

\section{Wheat}

Fructose and fructan significantly increased

Högy et al., 2009b

Hemicellulose reduced by $25.9 \%$ at elevated $\mathrm{CO}_{2}$

Porteaus et al., 2009 and low $\mathrm{N}$ supply, while under high $\mathrm{N}$ supply and elevated $\mathrm{CO}_{2}$, it reduced only $13 \%$; starch increased by $7-8 \%$ under elevated $\mathrm{CO}_{2}$ and low/high $\mathrm{N}$ supply conditions; water-soluble carbohydrate reduced by $7-15 \%$ at low/high $\mathrm{N}$ supply under elevated $\mathrm{CO}_{2}$

\section{Elevated $\mathrm{CO}_{2}$ and temperature}

\section{Kidney bean}

Elevated $\mathrm{CO}_{2}\left(700 \mu \mathrm{mol} \mathrm{mol}{ }^{-1}\right)$ and temperature Thomas et al., 2009 $\left(34 / 24^{\circ} \mathrm{C}\right)$ reduced glucose by $44 \%$, while high temperature alone increased sucrose and raffinose by $32.6 \%$ and $116 \%$, respectively

elevated $\mathrm{CO}_{2}$. For example, more waxes and extra layers of epidermal cells in leaves of plants under elevated $\mathrm{CO}_{2}$ may further reduce forage quality. Likewise, forage cuticles reduce microbial degradation of ingested forages (Owensby et al., 1996 and references therein). The major impact of lowered forage quality is that ruminants will have greater nutritional stress due to reduced intake and consequently lowered productivity (Craine et al., 2009).

Grasses with $\mathrm{C}_{3}$ photosynthetic pathway are more nutritious host plants than $\mathrm{C}_{4}$ grasses (Barbehenn et al., 2004 and references therein). However, $\mathrm{C}_{3}$ types in comparison to $\mathrm{C}_{4}$ are more adversely impacted by elevated $\mathrm{CO}_{2}$. The $\mathrm{C}_{3}$ types under elevated $\mathrm{CO}_{2}$ environments produce greater amounts of nonstructural carbohydrates, and have greater decline in their $\mathrm{N}$ than $\mathrm{C}_{4}$ types. Barbehenn et al. (2004) raised the issue of whether will $\mathrm{C}_{3}$ grasses remain superior to $\mathrm{C}_{4}$ under elevated $\mathrm{CO}_{2}$ levels. The experiment involving five species each of $\mathrm{C}_{3}$ and $\mathrm{C}_{4}$ grasses grown under $\mathrm{CO}_{2}$-enriched environments clearly demonstrated that a significant increase in sugars, starch and fructan in the $\mathrm{C}_{3}$ species under elevated $\mathrm{CO}_{2}$ was associated with a significant reduction in their protein levels, while protein levels in most $\mathrm{C}_{4}$ 
species being little affected by an elevated $\mathrm{CO}_{2}$. However, this differential response of the two types of grasses was insufficient to reduce protein in $\mathrm{C}_{3}$ to the levels of $\mathrm{C}_{4}$ grasses. Thus, Barbehenn et al. concluded that $\mathrm{C}_{3}$ grasses will remain more nutritious than $\mathrm{C}_{4}$ grasses at elevated $\mathrm{CO}_{2}$ concentrations, having higher levels of protein, nonstructural carbohydrates, and water, but lower levels of fiber and toughness, and lower total carbohydrate:protein ratios than $\mathrm{C}_{4}$ grasses.

To sum up, drought, heat and elevated $\mathrm{CO}_{2}$ will likely impact on forage and grain quality. However, their effects may be variable depending on crop growth stage, the duration and intensity of stresses, and soil N. Produce harvested from drought- or heat-stressed crops will have higher grain protein but of lower protein quality. Likewise, the produce from similar stresses will have reduced grain oil but improved oil quality. Drought and heat will also impact on grain minerals (both macro- and micronutrients); but to a variable extent. The lower nutritive value (reduced leaf $\mathrm{N}$ and protein) of the grasses and forages under $\mathrm{CO}_{2}$-enriched environments will have adverse impact on ruminants unless their diet is supplemented with more nutritive food. More importantly, genotypes responded differentially with respect to grain-quality attributes under drought and heat, which may provide researchers opportunities to identify germplasm or cultivars with least differences in grain quality under stress for direct cultivation or use in crop improvement programs to breed cultivars with no adverse effect of elevated $\mathrm{CO}_{2}$, drought and heat on grain quality.

\section{$j$ \\ 3. GLOBAL WARMING AND ALTERED PATHOGENS AND PESTS IMPACTS ON CROP PRODUCTION AND QUALITY}

Crop yield depends on growing-season weather, which also influences how pathogens and pests affect crops and their host-plant resistance. Plant pathogens account for 10-16\% of global food losses (Chakraborty and Newton, 2011), which amounts to about US $\$ 220$ billion annually (Ghini et al., 2008a). Although there is a paucity of information about the impact of climate change in plant pathogens and pest and their epidemics, its effects-which depend on changes in host distribution and phenology-will be noticed on their geographical spread, crop losses and plant protection options (Chakraborty et al., 2000a,b;Yáñez-López et al., 2012). Shaw (2009) further adds that there will also be changes in plant-associated microflora and direct biological effects on rapidly evolving pathogens. 
Insect pests may increase under drought while fungi will benefit from increased rainfall or due to changes in the temperature. Characterizing the dynamic interactions between changes in climate variables and their effects on pathogens and pests will therefore allow assessing their potential impacts on crops, trees and pastures, and to develop sound options for control, e.g. through genetic enhancement.

\subsection{Crop Pathogens and Pests in a Changing Climate}

Coakley et al. (1999) indicated that temperature seems to be the most important factor affecting insect ecology, epidemiology, and distribution, while plant pathogens are highly responsive to humidity and rainfall, as well as to temperature. $\mathrm{CO}_{2}$ may further promote the rapid establishment of invasive insect species (Zavala et al., 2008). Climate change will therefore affect the geographical and temporal distribution of pathogens and pests. For example, global warming, rainfall pattern changes, and new crop niches may lead to a significant change of crop health in Scandinavia (Roos et al., 2011).

Plant biomass production may increase as a result of a rise in $\mathrm{CO}_{2}$ concentration in the atmosphere. Proliferation of shoots, leaves, flowers and fruit provide more tissue that can also be infected by pathogens. Furthermore, sugar-dependent pathogens (e.g. rusts and powdery mildews) may increase as a result of rising carbohydrate content, whereas high canopy density and plant size can promote the growth, sporulation and spread of leaf infecting fungi such as rusts, powdery mildews and leaf necrotrophs, which require high air humidity but not rainfall (Ghini et al., 2008a). Likewise, necrotrophic pathogens will also benefit by an increase in the amount of crop residues. Chakraborty and Pangga (2004) indicated that about 26 plant pathogens may increase their severity in $\mathrm{CO}_{2}$-enriched environments.

\subsection{Plant Pathogen Scenarios under Climate Change}

Long-term datasets are important for determining scenarios for pathogens due to variation in $\mathrm{CO}_{2}$ levels, temperature, rainfall and other climate factors that affect their spread and severity (Jeger and Pautasso, 2008). For example, Evans et al. (2008) used 15 year multilocation data to develop and validate a weather-based forecasting model of phoma stem canker (Leptosphaeria maculans) epidemics on oilseed rape across the United Kingdom. They predict that phoma stem canker epidemics will increase in severity and spread northward by the 2020s. However, changes in pathogen ranges due to climate change are not yet well defined due to uncertainty 
for predicting the variables affecting them (Shaw and Osborne, 2011). Furthermore, primary data and historical records of many pathogens are often poor or lacking. Hence, Shaw and Osborne (2011) suggest that perhaps monitoring change and retaining the ability to innovate are needed for delineating adaptive responses. Likewise, as indicated by Chakraborty and Newton (2011), there has been limited field research on plant pathogens in the environments that realistically mimic climate change, which therefore reduces the assessment of options to enhance the adaptation of crops to emerging disease epidemics.

Although a lot of knowledge has been gathered about the changes in pathogen life cycle, expression of host-plant resistance, disease epidemiology and severity of disease epidemics, as well as pathogen inoculum production, there has not been enough research regarding the potential changes in pathogen biodiversity (Barbetti et al., 2012). New races or pathotypes will continue evolving and depending on crop husbandry and plant health management, but the changing climate may influence future changes in the distribution of emerging pathogen threat (Gregory et al., 2009). For example, as noted by Lake and Wade (2009), high $\mathrm{CO}_{2}$ may accelerate plant pathogen evolution, which could lead to changes in virulence. However, an increase in $\mathrm{CO}_{2}$ may not always affect disease incidence, as shown by experiments in phytotron for powdery mildew on grapevine (Pugliese et al., 2010) because of the increased photosynthetic activity of the host plants in hot environments with elevated $\mathrm{CO}_{2}$.

Chakraborty et al. (2008) provide a summary of the impacts of climate change (including the influence of elevated $\mathrm{CO}_{2}$ and $\mathrm{O}_{3}$ ) on main pathogens affecting crops and trees worldwide. They acknowledge the scarcity of knowledge in this area, which calls for generating more empirical data on host-pathogen biology under a changing climate. Details are given below for selected pests affecting major crops and trees, and whose severity and incidence may be influenced by climate change.

Irrespective of cereal rusts being an important subject of research, the lack of information on the potential effects of the changing climate on wheat rusts did not allow planning its appropriate forecast and management. Nevertheless, Chakraborty et al. (2011) by extrapolating the results from modeling other crop-pathogen interactions and using available research literature on wheat rusts indicated that the main risks brought by climate change will be increased grain yield loss, new pathotypes evolving faster, and, last but not least, the reduced effectiveness of host-plant resistance to rust pathogens. They further elaborate by indicating that an increase of 
biomass - due to high temperatures and elevated $\mathrm{CO}_{2}$ - could lead to an expansion of leaf area available for pathogen attack, which will enhance inoculum pressure. The changing weather may favor the spread of severe rust epidemics elsewhere, and to more conducive environments for increasing pathotypes evolution rates, thereby accelerating new virulence. Moreover, some of the known rust-resistance genes are affected by temperature and crop development, which may also be influenced by climate change. For example, emergence of an Sr31-virulent strain of stem rust (Puccinia graminis f. sp. tritici) in wheat, due to evolution of Ug99, sent an alarm bell to wheat researchers about the damaging effects of this pathotype to wheat production worldwide (Singh et al., 2008b). Likewise, there may likely be an increased prevalence of powdery mildew (Blumeria graminis f. sp. tritici) and scab (Fusarium spp.), and a decrease of stripe rust (Puccinia striiformis) affecting wheat production in China due to temperature increase (Yang et al., 1998).

Weather seems to be among the most significant factors affecting incidence, severity and the relative importance of Fusarium head blight or scab in wheat (Chakraborty and Newton, 2011). Warm and wet or humid environments at anthesis favor the pathogen, which causes blighting of the head, lowers yield and quality (e.g. shriveled kernels), reduces test weight and bread-making quality, and produces one or more mycotoxins. For example, scab epidemics will be more severe in the United Kingdom (especially in southern England) by 2050s due to climate change, which will also favor early wheat anthesis (Madgwick et al., 2011). Furthermore, scab severity may increase because, as a result of climate change, British farmers will grow more maize, whose debris will remain as a source of inoculum for Fusarium graminearum, which causes scab in wheat (West et al., 2012). Increasing rainfall will also favor wheat scab during the spring season in South America's Southern Cone (Ortiz, 2012).

The fungi Cochliobolus sativus (spot blotch) and Pyrenophora tritici-repentis (inducing tan spot) are the pathogens responsible for leaf blight in humid and hot areas, particularly in the Indo-Gangetic Plain, the major wheat production region in India (Ortiz et al., 2008a). Heat stress enhances their increasing severity with growth stage. Hence, wheat-bred germplasm adapted to heat and with host plant-resistance to leaf blight is needed to avoid grain yield loss in this bread-basket of the world. It can successfully be achieved by crossing cultigen resistance sources or CWR to high-yielding cultivars (Ortiz et al., 2008b).

Wheat head blast (Magnaporthe grisea, anamorph, Pyricularia grisea) is another emerging threat that may induce grain yield loss $>50 \%$ in warm 
(25-28 $\left.{ }^{\circ} \mathrm{C}\right)$, humid environments of South America's Southern Cone-one of the major grain baskets worldwide (Ortiz, 2011). Host-plant resistance to this pathogen should also become a priority target for wheat breeding under climate change.

Blast (Pyricularia oryzae) and sheath blight (Rhizoctonia solani) are among the major pathogens of rice worldwide. Kobayashi et al. (2006) found that rice plants grown under elevated $\mathrm{CO}_{2}$ concentration were more susceptible to leaf blast and sheath blight. Reduced leaf silicon content under elevated $\mathrm{CO}_{2}$ concentration leads to more susceptibility to blast disease, and the change in rice canopy structure (due to increased number of tillers under elevated $\mathrm{CO}_{2}$ concentration) may accelerate spread of sheath blight in the field.Thus, the potential risks to leaf blast infection and epidemics of sheath blight would increase in rice grown under elevated $\mathrm{CO}_{2}$ concentration (Kobayashi et al., 2006).

There is also potential risk that global warming, drought and high rainfall may lead to changes in pathogen severity and spread, adversely impacting legume production, especially in the developing world. For example, a higher incidence of dry root rot (Rhizoctonia bataticola) was noted in chickpea cultivars resistant to fusarium wilt (Fusarium oxysporum f. sp. ciceris) when temperatures exceeded $33^{\circ} \mathrm{C}$ in India, while epidemics of phytopthora blight of pigeonpea (Phytophthora dreschsleri f. sp. cajani) over the last decade was attributed to high intermittent rainfall $(>300 \mathrm{~mm})$ during the crop season in India (Savary et al., 2011 and the references therein). Likewise, Chakraborty et al. (2000a,b) observed an increased aggressiveness and fecundity of Collitotrichum gloeosporiodes, the causal agent of anthracnose in tropical legumes such as Stylosanthes.

Potato leaf blight (Phytophthora infestans) is the most damaging disease of potato worldwide. It is predicted that warming temperature and humidity will increase leaf blight, expanding its range above $3000 \mathrm{~m}$, where it is absent today in the Andes region of South America (Ortiz, 2012). The suitability for producing high-quality coffee, such as the acidic Arabica, will be affected by heat, which also favors some pathogens such as Hemileia vastatrix causing coffee rust (Ortiz, 2012).

Climate change may also adversely impact host-plant resistance in banana and plantains. For example, scenario analyses suggest that the favorable period for the development of black leaf streak (or black Sigatoka) (Mycosphaerella fijiensis) and other foliar plant pathogens affecting bananas may be reduced (de Jesus Júnior et al., 2008). Black leaf streak of banana and plantain may decrease in producing locations of Central America and 
other coastal areas in the American continent because a switch toward unfavorable environments for the pathogen (low relative humidity and rainfall affect adversely its development) despite high temperatures, which are often associated with increased diseases pressure. Nonetheless, there will be extensive areas favoring the occurrence of this disease, especially in environments that favor disease development (Ghini et al., 2007).

Climate change will affect also pathogens affecting forests, as already noted for annual and perennial crops. Europe expects to witness an increased incidence and severity of pathogens, which-due to rising temperaturemay move northward in this continent (La Porta et al., 2008). Sturrok et al. (2011) suggest, based on their North American analysis for yellow-cedar and sudden aspen declines, that a strategy for managing emerging pathogen and pest threats due to climate change should include monitoring, forecasting, planning and mitigation. They also highlight that the uncertainty brought by climate change to forests can be reduced by research and risk assessment, and through linking them to policy, planning and decision making.

\subsection{Emerging Changes in Pest Dynamics under Climate Change}

A range of insect and nematode pests cause substantial damage to crops production and quality. Many of these pests are also carriers of viruses that cause significant losses to production. As for pathogens, the changes in climatic conditions may cause some insect and nematode pests to expand its range into new areas or retract from the areas wherein these pests currently pose a serious threat to crops production or altogether a new virulent biotype may emerge. The present day evidence suggests that the insect pests including lepidopteron respond to warming-from changes in phenology and distribution to undergoing evolutionary changes albeit at the population level in Europe and North America (Menéndez, 2007). Growing plants in elevated $\mathrm{CO}_{2}$ generally increases the carbon-to-nitrogen (C:N) ratio of plant tissues (Hamilton et al., 2005) and reduces the nutritional quality (Coviella and Trumble, 1999). As a result the insects may increase their food intake to compensate for leaf nitrogen content (Coviella and Trumble, 1999; Holton et al., 2003). Exposure to elevated $\mathrm{CO}_{2}$ has been reported to increase the loss of leaf area by foliage chewer, western corn rootworm (Diabrotica virgifera) and phloem feeder aphids (Aphis glycines) in soybean (Dermody et al., 2008). This increased loss of leaf area to these herbivores is associated with increased C:N ratio and leaf surface temperature (Dermody et al., 2008). Further research has shown that increased susceptibility of 
soybean to western corn rootworm and Japanese beetle (Popillia virgifera) under elevated $\mathrm{CO}_{2}$ concentrations is associated with the reduced expression of genes related to the jasmonic acid (JA) pathway (Zavala et al., 2008). Global warming may result in the breakdown of resistance to certain pests, for example, sorghum cultivars exhibiting resistance to sorghum midge (Stenodiplosis sorghicola (Coq.)) in India become susceptible to this pest under high humidity and moderate temperatures near the equator in Kenya (Sharma et al., 1999).

Insects may also appear in the region(s) where they are not currently known. For example, potato tuber moth (Phthorimaea operculella), which at present is restricted to coastal areas and inter-Andean valleys, may expand its range above $3000 \mathrm{~m}$, where it is absent today in the Andes region of South America, due to climate change (Ortiz, 2012).

Root-knot nematode (Meloidogyne incognita) infects large number of crops including tomato, causing severe losses in yield. Salicylic acid (SA) and JA are the major signaling pathways of plant response to nematode infection (Vasyukova et al., 2003; Soriano et al., 2004). Sun et al. (2011) studied the effects of elevated $\mathrm{CO}_{2}$ on nematode-induced defense response in tomato genotypes differing in the JA pathway. Their study revealed that elevated $\mathrm{CO}_{2}$ reduces the JA-pathway (a JA-defense-dominated genotype) defense against M. incognita in the wild type and in a genotype in which defense is dominated by the JA pathway but upregulates the SA-pathway defense in the wild type and in a JA-defense-recessive genotype (jasmonate-deficient mutant), which means that $\mathrm{CO}_{2}$-induced changes of plant resistance may lead to genotypespecific response of plants to nematode under elevated $\mathrm{CO}_{2}$.

There will be changes in the spatial distribution of races of root-knot nematodes and leaf miner (Leucoptera coffeella) affecting the coffee crop in Brazil (Ghini et al., 2008b; Haggar and Schepp, 2011). An increase in infestation of both pests will likely be due to a higher number of generations per month than before.

\subsection{Adapting Crops to Emerging Pathogens and Pests}

Adaptation to climate change, which depends on local conditions, will therefore consider integrated plant health management for existing and emerging pathogens and pests. Such an approach will require a set of tools such as models for predicting potential geographical distribution, seasonal phenology, and population dynamics at a range of spatial and temporal scales (Sutherst et al., 2011). Crop-pest models offer the means for designing sound plant health management of pathogens and pests. 
Host-plant resistance will continue to be very important for plant health management under climate change because rising temperature and variation in humidity may favor emerging pathogen and pest epidemics. Likewise, plant breeding for host-plant resistance to pathogens and pests leads to fewer pesticide sprays, which also means a reduction in fuel use and lowering $\mathrm{CO}_{2}$ emissions (Ortiz, 2011), thereby mitigating climate change.

\section{MANAGEMENT AND PREVENTION OF AFLATOXIN}

At present, over 5 billion people in the developing world are regularly exposed to mycotoxins, especially aflatoxins, through consumption of contaminated staple food. Moreover, the agricultural produce contaminated with mycotoxins drastically limits the access of producers to the global markets, which have set high standards for food safety. Mycotoxins in animal feeds also represent high risk to the growth of livestock and trade of feed (Wu et al., 2011a and references therein). Mycotoxins of worldwide importance include aflatoxins $\left(B_{1}, B_{2}, G_{1}\right.$, and $\left.G_{2}\right), D O N$, zearalenone, fumonisin $\mathrm{B}_{1}, \mathrm{~T}-2$ toxin, and ochratoxin $\mathrm{A}$, produced by fungi on pre- and postharvest foods and feeds.

The fungi Aspergillus flavus and Aspergillus parasiticus colonize maize and nuts including peanut, with former fungi producing aflatoxin $B_{1}$ and $B_{2}$ while the latter producing $B_{1}, B_{2}, G_{1}$, and $G_{2}$. Aflatoxin $B_{1}$ is the most toxic of aflatoxins. Aflatoxins in food can cause death, impair growth and development of children, suppress the immune system, enhance hepatitis B virus and hepatitis $\mathrm{C}$ virus infection, increase risk to certain types of cancer by several fold, and impede the uptake and utilization of micronutrients in humans and livestock (Lewis et al., 2005; Fokunang et al., 2006; Liu and Wu, 2010; Wu et al., 2011a and references therein). It is beyond the scope of this section to discuss in greater detail about the various mycotoxins and associated fungi and their control measures instead we discuss various aspects of aflatoxin research and management options that lead to minimize the risk of aflatoxin contamination under a changing climate in maize and peanut, the two food crops highly susceptible to aflatoxin.

\subsection{Modeling Climatic Risks to Aflatoxin Contamination}

How does climate change impact food safety, in addition to food security (Battisti and Naylor, 2009; Ronald, 2011; Vermeulen et al., 2011), has recently been dealt elsewhere (Cotty and Jaime-Garcia, 2007; Tirado et al., 2010; Paterson and Lima, 2010, 2011; Magan et al., 2011). Essentially, 
the biggest risk with respect to mycotoxins from climate change will be found in the developed world with temperate climates, for example, in areas of Europe and the USA as these regions will become warmer reaching temperature of $33{ }^{\circ} \mathrm{C}$, close to the optimal for aflatoxin production. Currently, in very cold climates aflatoxins may not be of any significantly greater concern than exists where even global warming will not result in optimal temperatures for Aspergillus growth. The tropical climates, and if the temperature continue to rise as predicted by various IPCC reports and exceed $40^{\circ} \mathrm{C}$, may become too inhospitable for conventional fungal growth and mycotoxin production. The warmer weather, heat waves, greater precipitation and drought in tropical climates will have greater impact on mycotoxins, and this subject has been discussed in greater detail by Paterson and Lima (2011) for Africa, Europe, Asia, Latin America and North America with respect to the risk of mycotoxins contamination in food and feed crops. They also highlighted that crops introduced to exploit altered climate may be subject to fewer mycotoxins producing fungi. The increased mycotoxins and UV radiation may cause fungi to mutate on crops and produce different mycotoxins. Crops when subjected to drought and high temperature, especially during reproductive phase, are under greater risk to aflatoxin contamination by A.flavus and $A$. parasiticus, with even greater risk to aflatoxin during the storage.

Water activity $\left(a_{\mathrm{w}}\right)$ and temperature, and their interactions with aflatoxin gene cluster significantly impact fungal growth and biosynthesis of aflatoxin; and gaining knowledge on these interacting factors should facilitate to forecast and develop effective control strategies to minimize the risk of aflatoxin contamination in food and feed crops (see Section 4.5; Miraglia et al., 2009; Magan et al., 2011).

Using agricultural production systems stimulater (APSIM) version 5.1 (aflatoxin risk simulation model; Wright et al., 2005), the weather and the peanut yield data at Kingaroy from 1890 to the present time in South East Queensland, Australia, Chauhan et al. (2008a) showed an 11.7\% reduction in peanut pod yield since 1980 . The risk of significant aflatoxin contamination, which was one in 11 years until 1979, increased to one in 3 years thereafter. They relate this increase to the changes in climate that indicated that since 1980, when changes in the risk became more noticeable, inseason crop rainfall decreased by $8 \%$, maximum temperature increased by $2.1 \%\left(0.6^{\circ} \mathrm{C}\right)$ and minimum temperature by $7.4 \%\left(1.1^{\circ} \mathrm{C}\right)$, while radiation remained unchanged. Thus, risk to aflatoxin contamination in peanut could be minimized by growing early maturity cultivars, as well as through a late planting strategy to avoid high temperatures during the pod-filling stage. 
Boken et al. (2008) are probably the first to use advanced very highresolution radiometer satellite data and crop simulation models to predict aflatoxin contamination in peanut in Mali. Using the normalized difference vegetation index (NDVI) averaged for the reproductive phase of peanut, to examine the relationship with annual peanut yield, an indicator of drought and aflatoxin, they found that AVHRR-based NDVI at reproductive phase is moderately correlated $\left(R^{2}=0.56\right)$ with peanut yield, which could be used to predict drought. The aflatoxin measured in peanut samples collected from various locations across Mali were found to be related to the NDVI, total precipitation, and maximum temperature averaged over the reproductive phase of peanut, which may be considered as potential variables to monitor and predict aflatoxin contamination. Predicting aflatoxin incidence will help identify risk zones to segregate the aflatoxin-contaminated peanuts from distribution among the general public.

High temperatures and end-of-season drought have been found to be associated with increased risk of aflatoxin contamination in peanut (Craufurd et al., 2006; Cotty and Jaime-Garcia, 2007). Chauhan et al. (2010) used APSIM peanut module to investigate the four temperature-response functions at fractional available water $<0.20$ and the crop in later development of the pod-filling stage to develop aflatoxin risk index (ARI). The ARI explains $95 \%$ of the variation in aflatoxin contamination (varied from 0 to $800 \mu \mathrm{g} \mathrm{kg}^{-1}$ ) in several Australian tropical and subtropical environments and $96 \%$ of the variation in the proportion of aflatoxin-contaminated loads of peanuts in the Kingaroy, Australia during eight seasons of evaluation. The simulation of ARI using climatic data from 1890 to 2007 indicated a threefold increase in its value since 1980 compared to the entire previous period, which they found to be associated with increases in ambient temperature and decreases in rainfall. The ARI predicted, using this interface for eight growers, correlated significantly with the level of contamination in the crop, suggesting that ARI is a reliable indicator of aflatoxin contamination that can be used in aflatoxin research as well as a decision-support tool to monitor preharvest aflatoxin risk in peanuts.

Maize has been the most extensively studied crop among cereals to predict occurrence of mycotoxins. Chauhan et al. (2008b) developed a similar model to quantify climatic risks of aflatoxin contamination in maize. The model performed well in simulating climatic risk of aflatoxin contamination in maize as it explained $69 \%$ and $62 \%$ of the variation, respectively for a range of rainfed and irrigated Australian locations. In further evaluations, the risk of aflatoxin contamination in four nonirrigated maize-growing 
locations of Queensland using 106 years of climatic data, the model revealed that locations with dry and hot climates had a much higher probability of higher aflatoxin risk compared with locations having dry or hot conditions alone. This finding suggests that under nonirrigated conditions, the risk of aflatoxin contamination could be minimized by adjusting sowing time or selecting an appropriate hybrid to better match the grain filling period to coincide with lower temperature and water-stress conditions. More recently, Wu et al. (2011b) used temperature, rainfall, changes in insect population dynamics, and agronomic factors to predict fungal growth and mycotoxin risks in maize in the USA. The predictions revealed that if the current climate patterns continue in twenty-first century, it is predicted that aflatoxin and fumonisin concentrations in maize will likely increase, whereas DON levels will decrease. However, climate change-induced alterations in cropping patterns or shifts in pathogen populations may create new opportunities for DON risk in the areas where maize is currently not grown or is a minor crop, and where new, more aggressive isolates of $F$ graminearum occur.

Climate databases created under the North American Regional Climate Change Assessment Program (NARCCAP) (www.narcap.ucar.edu), which uses four international global climate models and six regional climate models, provide ranges of climatic conditions until 2070 - day and night temperatures, number of days/nights above specific temperatures, precipitation, relative humidity, and soil moisture - that could be used to assess how future climatic scenarios may affect toxin levels in crops around the world.

\subsection{Geostatistics and Geographic Information Systems to Monitor Spatial Variability in Aflatoxin}

Geographic information systems (GIS) are capable of assembling, storing, manipulating, and displaying data georeferenced using geographic coordinates (latitude and longitude). Geostatistics provides a set of tools useful in characterizing variability in space. Both GIS and geostatistics can be used to describe, analyze and show the spatial distribution of several variables to define the cause-effect relationships of various variables with their geographic position (Nelson et al., 1999). The various reports to-date suggest that there has been increased use of geostatistics, GIS and satellite imagery data in agriculture (Lewis et al., 1998; Boken et al., 2008; Shamseddin and Adeeb, 2012; Kogan et al., 2012). Geostatistics has been used to characterize spatial and temporal variability at regional levels to predict and monitor shifts in community structure of aflatoxin-producing fungi, A. flavus (Orum et al., 1999). Such information will be helpful to 
develop control strategies directed toward changing the composition of fungal communities.

The two major strains in A.flavus, based on sclerotial morphology, are $\mathrm{S}$ and $\mathrm{L}$ strains, with the former producing sclerotia $<400 \mu \mathrm{m}$ in diameter, and the latter $>400 \mu \mathrm{m}$ in diameter (Cotty, 1989). The S-strain isolates produce high levels of aflatoxin $\mathrm{B}_{1}$, whereas the $\mathrm{L}$-strain isolates are more variable in aflatoxin production (Cotty, 1997). These two strains also differ in production of the type of aflatoxins, with $\mathrm{L}$ isolates producing only aflatoxin $\mathrm{B}$ while the S isolates produce both aflatoxin B and G (Cardwell and Cotty, 2002). Populations of both strains comprise numerous subpopulations known as vegetative compatibility groups (VCGs) (Horn and Greene, 1995). Isolates in the same VCG have the same alleles for all compatibility loci, and when paired hyphae fuse to form heterokaryons (Papa, 1986). Isolates in different VCGs differ in sclerotial size, mating type, aflatoxin production, and intraspecific aflatoxin inhibition, while those from the same VCG have similar sclerotial size and mating type and produce the same kinds of mycotoxins (Horn and Greene, 1995; Mehl and Cotty, 2010; Grubisha and Cotty, 2010). Regional differences in aflatoxin contamination of crops may be attributed to climatic conditions and to agricultural practices that increase the susceptibility of plants to invasion by $A$. flavus. For example, it is well known that drought stress accompanied by elevated temperatures at seed development promotes $A$. flavus invasion and aflatoxin contamination in cotton, maize and peanut. Surveys of $A$. flavus isolates from various geographic regions have revealed differences in strain composition and in aflatoxin contamination. Using geostatistical analysis, Jaime-Garcia and Cotty (2003) detected recurrent patterns of high and low aflatoxin in commercial cotton seeds produced in South Texas, USA with greatest contamination occurring from the Central Coastal Bend region through the southern Upper Coast region, whereas, the Rio GrandeValley region experiences the least contamination. Likewise, the regional patterns of differences in aflatoxin contamination were also reported in peanut. For example, $62-94 \%$ of isolates from western Texas, Georgia and Alabama produced at least $10 \mu \mathrm{g}$ of aflatoxin $\mathrm{B}_{1} \mathrm{ml}^{-1}$, while the isolates producing in excess of $10 \mu \mathrm{g}$ of aflatoxin $\mathrm{B}_{1} \mathrm{ml}^{-1}$ from central Texas, Virginia and North Carolina ranged from 0\% to 52\%; with other isolates often atoxigenic in nature (Horn and Dorner, 1999).

The Republic of Benin in West Africa has four agroecological zones: the coastal Savanna (CS), the southern Guinea Savanna (SGS), the northern Guinea Savanna (NGS) and northernmost Sudan Savanna (NSS). All of them differ in latitude, precipitation and temperature. The latitude 
differences among these regions relate to the differences in precipitation and rainfall pattern (bimodal or unimodal) and temperature, with decreasing rains and increasing temperatures noted as the latitude increase-from humid equatorial tropics in the south to the dry savanna near the Sahel in the north. When investigated, the pattern of distribution of Aspergillus across the four agroecological zones in Benin, Cardwell and Cotty (2002) found that all soil samples contained A. flavus. However, their densities differ from $<10$ to $>200 \mathrm{CFU} \mathrm{g}^{-1}$ soil. Furthermore, they detected no yearly variation in CFU counts or significant change with cropping systems within a zone, but differed significantly among zones, with greater number of CFU of $\mathrm{L}$ strains in southern latitudes and higher numbers of CFU of S strains in the northern latitudes. These two strains also differ in their distribution and aflatoxin-producing ability. All S strains produce both B and $\mathrm{G}$ aflatoxins, while, only $44 \%$ of the total $\mathrm{L}$ strain detected from the soils produce aflatoxin B. Aspergillus parasiticus and $A$. tamarii, though present in $<10 \%$ of the fields, were not particularly associated with any of the four agroecological zones. More prevalence of $\mathrm{S}$ strains is also reported from regions characterized by low rainfall and high temperature in North America (Horn and Dorner, 1999; Orum et al., 1999), which probably is the inherent ability of S strains to produce more small sclerotia as the survival mechanisms to adapt to rapid and extreme fluctuations in moisture and temperature.

Battilani et al. (2008) used aridity index (AI), derived from meteorological data, to estimate the probability of aflatoxin $\mathrm{B}_{1}$ contamination in maize running a logistic regression. They found that AI as a good index to summarize meteorological conditions in relation to aflatoxin $\mathrm{B}_{1}$ contamination in maize. In $64 \%$ of the cases, it was found that the prediction about aflatoxin $\mathrm{B}_{1}$ contamination was accurate, while the prediction in $23 \%$ cases was overestimated, and underestimated in $13 \%$ cases. Such predictions may provide opportunity to maize growers plan maize management preand postharvest or segregate the produce either safe or unsafe for human consumptions based on the probability of the occurrence of aflatoxin contamination. Clearly, most of this research using geostatistics and GIS tools is needed to assess the risks of aflatoxin contamination, which in the long run should lead to the identification of regions or cropping systems leading to the production of food free from mycotoxins.

\subsection{High-Throughput and Cost-effective Assays to Detect Aflatoxin}

A wide variety of aflatoxin-monitoring technologies is available to detect and quantify aflatoxin levels (Pascale, 2009; Margos and Busman, 2010). 
Conventional analytical methods for mycotoxin include thin-layer chromatography (TLC), high-performance liquid chromatography (HPLC), and gas chromatography (GC). In recent years, significant improvement has been introduced in the analysis of aflatoxins especially using column chromatography technology (Buttinger, 2010). The use of packaging materials with particle sizes below $2 \mu \mathrm{m}$, and the use of chromatograph which can support very high pressure, led to dramatic reduction in the analysis time. More recently, Medina and Magan (2012) compared the performance of chromatography columns with particles of 5 and $3 \mu \mathrm{m}$ with the new $2.7 \mu \mathrm{m}$ solid core particles for the analysis of aflatoxins using trifluoroacetic acid precolumn derivatization. The results of the comparisons revealed that shorter columns $(100 \times 4.6 \mathrm{~mm})$ with new solid-core particles in comparison to traditional columns are suitable for the analysis of mycotoxins. This modification reduced the analysis time by $45.5 \%$ and $33.3 \%$ with respect to columns with particle size $5 \mu \mathrm{m}(150 \times 5.6 \mathrm{~mm})$ and $3 \mu \mathrm{m}(150 \times 5.6 \mathrm{~mm})$ respectively, without any detrimental effect on performance. This had lead to a reduced analytical cost, and increase in the total number of samples that can be analyzed; i.e. from 57 to 80 samples for columns with particle size of 5 and $3 \mu \mathrm{m}$ respectively, to up to 111 samples day ${ }^{-1}$. In spite of all these technological advances and saving in analytical cost and increasing throughput, the use of such assay in the developing countries is still limited due to high cost, difficulties with importation, and the lack of appropriate laboratory facilities and well-trained staff. Immunological methods are preferred over the analytical methods because of their simplicity and costeffectiveness. However, commercial kits based on immunological methods are expensive, and there may be difficulties in their import. Waliyar et al. (2009) developed a simple and inexpensive competitive enzyme-linked immunosorbent assay (cELISA) that has lower detection limit $\left(1.0 \mu \mathrm{g} \mathrm{kg}^{-1}\right)$ and costs (about US $\$ 1$ per sample) less than other available methods, with high-throughput efficiency (>100 samples per day). This cELISA test has provided a unique opportunity to researchers in the developing world to select breeding populations possessing resistance to aflatoxin contamination, and to evaluate food, feed and related commodities for aflatoxin contamination. This technology has been successfully transferred and adopted by several laboratories in India, Mozambique, Kenya, Malawi and Mali, and is contributing to the quality certification of the farmers produce, and enhancing the competitiveness of the produce in domestic and international markets. For example, using this technology, Malawi has revived its large-seeded graded peanut export to Europe and South Africa, benefiting her farmers. Likewise, Kumar and Bandyopadhyay (2012) also developed a cost-effective, 
high-throughput (up to 200 samples per day), simple and sensitive assay, called Afla-ELISA - for quantitative determination of aflatoxins- with the

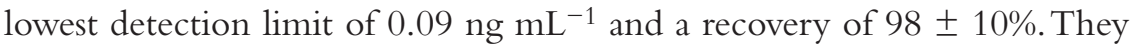
are advocating its use to monitor aflatoxins in maize in West Africa (Kumar and Bandyopadhyay, 2012).

\subsection{Atoxigenic Fungal Strain as Biocontrol Agent to Manage Aflatoxin Contamination in Crops}

To date, a number of atoxigenic isolates of $A$. flavus have been reported, of which, few are commercially used as biopesticide to control aflatoxin contamination in cotton, maize and peanut (see below within this section). The $A$. flavus genome has been sequenced $(36.3 \mathrm{Mb})$ and contains 13,091 predicted genes in eight chromosomes (http://www.aspergillusflavus.org/). Twenty-five genes clustered within a $70 \mathrm{~kb}$ DNA region are involved in aflatoxin biosynthesis, and the functions of 19 genes are known (Yu et al., 2004). Knowledge of the molecular mechanisms for the loss of aflatoxin production in atoxigenic $A$. flavus may help develop assays for rapid detection of atoxigenic isolates to plan a better biocontrol strategy by including mixture of genetically different atoxigenic isolates to provide effective control of aflatoxin contamination in food crops. Using aflatoxingene-specific primers, Chang et al. (2005) grouped the 38 atoxigenic A. flavus isolates from the southern USA into eight deletion patterns, which appear to be diverse but not rare. They found genetic drift as the probably driving force for the loss of the entire aflatoxin gene cluster in atoxigenic isolates. A detailed analysis of two atoxigenic isolates commercially used as biopesticide further reveals that AF36 (used as biocontrol agent in cotton in the USA) has a defective polyketide synthase gene required for aflatoxin biosynthesis (Ehrlich and Cotty, 2004), while the NRRL 21882 (used as biocontrol agent in peanut and maize in the USA) lacks the entire aflatoxin biosynthesis gene cluster (Chang et al., 2005). Chang and Hua (2007) also found a defective polyketide synthase gene in TX9-8, which competitively prevented aflatoxin accumulation by $A$. flavus isolates producing varying levels (low to high) of aflatoxin. More recently, Chang et al. (2012) found that a lack of production of aflatoxin in A.flavus isolate K49 (NRRL30797) relates to single nucleotide mutations in the polyketide synthase and hybrid polyketide-nonribosomal peptide synthase genes.

Molecular analysis of the 35 atoxigenic A. flavus isolates collected from peanut fields in China reveals that 24 of these isolates containing no detectable aflatoxin had the entire aflatoxin gene cluster, while 11 had five 
different deletion patterns in aflatoxin gene cluster (Yin et al., 2009). Likewise, Criseo et al. (2008) investigated deletions in aflatoxin gene cluster among 134 natural atoxigenic A. flavus isolates, and found deletion as a cause of loss of aflatoxin-producing ability in 84 isolates. Jiang et al. (2009) detected $89.59 \mathrm{~kb}$ deletion in the aflatoxin gene cluster in atoxigenic strain A051, which is replaced by a $3.83 \mathrm{~kb}$ insert located at 300 bp upstream ver 1 gene and 2594 bp downstream a putative gluconolactone oxidase gene. Donner et al. (2010) detected deletion of varying size among six of the 18 atoxigenic A.flavus isolates from Nigeria, while the remaining 12 isolates had all the aflatoxin-pathway genes, but with defects. These findings suggest that deletions within the aflatoxin gene cluster in some atoxigenic $A$. flavus isolates are very common, but diverse; and the analysis of deletion pattern within aflatoxin gene cluster would be an effective method for the rapid identification of atoxigenic isolates for developing biocontrol agents. The exact mechanisms for the loss of aflatoxin production in some atoxigenic A. flavus isolates with no deletion in their aflatoxin gene cluster indicate that there must be other mechanisms responsible for the loss of aflatoxin production in these isolates, which is the subject for further investigation.

Recently, Degola et al. (2011) developed a simple and an inexpensive procedure to scale up the screening process employing a 96-well microplate (or with 380-well microplates based on preliminary work) for evaluating high numbers of pairings of $\mathrm{afla}^{-} / \mathrm{afla}^{+}$strains. A visual observation of fluorescence in the wells allowed them to detect a positive signal down to $250 \mathrm{ppb}$; and the processing of the digital image of the microplate has been effective in detecting aflatoxin concentrations as low as 50-100 ppb, which correlates well with those obtained using a microplate fluorescence reader or using an ELISA immunoassay. This assay requires small volumes $\left(200 \mu \mathrm{L}\right.$ well $\left.{ }^{-1}\right)$ of inexpensive medium and 4 days of incubation at $28{ }^{\circ} \mathrm{C}$ is sufficient to detect aflatoxin production even with "slow" mycotoxin producer inoculated with $10^{4}$ conidia (Degola et al., 2009).

Competitive exclusion of aflatoxin producers by atoxigenic strains of A. flavus is a viable option for aflatoxin management. Biological control is based on this idea of competitive exclusion whereby a large population of atoxigenic strains of A.flavus is established in the soil that outcompetes toxigenic strain, which results in reduced concentration of aflatoxin. Essentially, the introduced atoxigenic strains have the ability to compete and overtake native populations because of their competitive ability to occupy ecological niches and deprive the native populations for space and essential nutrients (Abbas et al., 2011a,b and references therein). 
Huang et al. (2011) investigated how the competition suppresses the fungus' ability to infect or produce aflatoxin when challenged. They found that an unknown signaling pathway is initiated in the toxigenic strain by physical interaction with an appropriate atoxigenic strain in the first $24 \mathrm{~h}$, which prevents or downregulates normal expression of aflatoxin. These authors termed thigmo-downregulation of aflatoxin synthesis as the mechanistic basis of intraspecific aflatoxin inhibition and the major contributor to biological control of aflatoxin contamination. Furthermore, the timing of host contact (i.e. atoxigenic strain making host contact prior to aflatoxin producers) is as important for the competition during disease cycles as is the innate competitive ability (Mehl and Cotty, 2011).

The two major atoxigenic strains of $A$.flavus used as biopesticide in biological control of aflatoxin in the USA are AF36 (NRRL 18543) in cotton (U. S. Environmental Protection Agency, 2003) and NRRL 21882 (AflaGuard $\left.^{\circledR}\right)$, which was initially used in peanut and later on in maize as well (U. S. Environmental Protection Agency, 2004; Dorner, 2009a,b; Abbas et al., 2011a,b). Few more atoxigenic A. flavus strains reported in the literature are K49 (NRRL 30797) (Abbas et al., 2006), TX9-8 (Chang and Hua, 2007), AF 051 (Jiang et al., 2009), TOب (Degola et al., 2011), and isolate 51 (Huang et al., 2011).A recent in vitro study using toxigenic and atoxigenic strains, in pairs, led researchers to identify additional atoxigenic $A$.flavus strains such as NRRL 50427, NRRL 50428, NRRL 50429, NRRL 50430, and NRRL 50431, which are more effective than NRRL 21882 for the biocontrol of aflatoxin in peanut (Horn and Dorner, 2011). However, large-scale field trials have been suggested to evaluate the effectiveness of these strains under field conditions as the in vitro aflatoxin inhibition does not always reflect the true effectiveness of the strain in controlling aflatoxin contamination in developing crops (Cotty and Bhatnagar, 1994). Moreover, the inoculum rate, the carrier of inoculum used, the method (delivery) and timing of inoculum application significantly impact the success of the biological control of aflatoxin in the field (Dorner, 2009a,b; Lyn et al., 2009; Abbas et al., 2011a). For example, a direct spray application of atoxigenic A. flavus strain is better than soil inoculation in controlling maize aflatoxin contamination, and that a water-dispersible granule is a viable delivery system for maintaining viability and efficacy of the biological control agent K49 (Lyn et al., 2009). In the case of peanut, it is recommended that the inoculum be applied at $22.5 \mathrm{~kg} \mathrm{ha}^{-1}$ in the field at between 60 and 80 days after planting when the foliage canopy is well developed and at a time when enough soil moisture is available (Dorner, 2009a). 
AF36 has so far been applied in over 50,000 ha to control aflatoxin contamination in cotton in the US southwestern (Das et al., 2008). AflaGuard $^{\circledR}$ is now commercially used to prevent aflatoxin contamination in peanut in the USA.The Afla-Guard ${ }^{\circledR}$ has so far been applied approximately in 2000 ha in the states of Alabama and Georgia to test its efficacy in controlling the aflatoxin in peanut, which resulted in mean reduction in aflatoxin concentration by $85 \%$ in the treated peanuts (Dorner, 2009a). Similar package has also been developed, and is in use to control aflatoxin contamination in peanut in Australia (Pit and Hocking, 2006).

Of late, there has been systematic effort to identify atoxigenic strain among the native $A$. flavus populations to control aflatoxin contamination in maize in West Africa. Atehnkeng et al. (2008) investigated 11 naturally occurring atoxigenic isolates of $A$. flavus to reduce aflatoxin contamination in maize in Nigeria. They detected two strains from Lafia, La 3279 and $\mathrm{La} 3303$, as the most effective in reducing aflatoxin concentrations in both laboratory and field trials. In a 2 year field study, La 3279 was the most effective isolate that reduces aflatoxin contamination by $99 \%$, while La 3303 could provide up to $92 \%$ reduction in aflatoxin contamination in maize. These two endemic strains from the region are well adapted to West African environments and thus, will not have any regulatory issue over their use as biopesticide for the biocontrol of aflatoxin contamination in maize throughout the region. Furthermore, Donner et al. (2009) found significant differences in $A$. flavus distribution in maize fields, sampled across three agroecological zones in Nigeria. They detected significantly greater proportions of atoxigenic A. flavus strains in the Northern Guinea Savanna than in the Southern Guinea Savanna or from Derived Savanna zones of Nigeria. This finding suggests that it is possible through systematic surveys to identify regions within a country that are predominated by atoxigenic A. flavus strains as safe regions to minimize aflatoxin contamination in food crops. Atehnkeng et al. (2010) evaluated the efficacy of a mixture of four atoxigenic strains in the field for reducing aflatoxin contamination through displacement of aflatoxin producers in maize, using sterile sorghum grains as carriers of atoxigenic strains, in Nigeria. In 2 years of evaluation, they found that $67-95 \%$ reduction in aflatoxin, which was associated with $74-80 \%$ displacement of aflatoxin producers. These results demonstrate that effective atoxigenic strains native to West Africa can be selected from fungal communities associated with maize production, and successfully utilized to minimize the risk of aflatoxin exposure in human populations. Another study in the central Republic of Benin also revealed the natural occurrence of 
atoxigenic strain BN030D. BN030D release greatly increases its population, leading to its spread in both time and space, thus, it is a promising candidate for the development of competitive displacement strategies for the control of aflatoxin in maize in the Republic of Benin (Klueken et al., 2009). More recently, Probst et al. (2011) evaluated the aflatoxin-producing potential of the 290 A.flavus strains from Kenya; and they identified 12 most effective strains that could reduce aflatoxin levels above $80 \%$ in maize in Kenya. The reduction in aflatoxin levels by these selected atoxigenic strains are comparable to those reported with a strain from USA (NRRL-21882), which is used commercially for aflatoxin management in maize and peanut. Hence, these atoxigenic strains have potential to biologically control within highly toxigenic A.flavus communities associated with maize production in Kenya. Probst et al. (2011) further noted that these strains belong to different VCGs, which may provide opportunity to utilize VCG mixtures that may compete effectively in a greater diversity of environmental niches than individual strains, similar to those reported for West Africa (Atehnkeng et al. 2010).

Abbas et al. (2011b) assessed the competitiveness of three atoxigenic strains (K 49, NRRL 21882 and AF 36) to displace toxigenic strains (K 54 and F3W4) in maize. Their study revealed that K 49 and NRRL 21882 are superior to AF 36 in reducing total aflatoxin contamination. Neither K 49 nor NRRL 21882 produce cyclopiazonic acid (CPA), another form of mycotoxin, however, when challenged with toxigenic strains, both had similar effect on reducing the CPA and aflatoxins (84-97\% with K 49 and $83-98 \%$ with NRRL 21882). In contrast, AF 36 reduces aflatoxins by $20 \%$ using F3W4 and 93\% with K 54, but there was no reduction in CPA by F3W4 and only a 62\% reduction in CPA with $\mathrm{K} 54$. These results indicate that K 49 is as effective as NRRL 21882 is in reducing both aflatoxins and $\mathrm{CPA}$ in maize.

For biological control to remain effective, it is essential that atoxigenic isolates should belong to VCG that do not have toxigenic members to ensure that atoxigenic and toxigenic strain within a VCG do not exchange genetic material and generate progenies that produce aflatoxin (Ehrlich et al., 2007), which makes the use of these strains for biological control agent ineffective in minimizing aflatoxin contamination in crops. The recent discovery of sexual cycle in atoxigenic fungi may alter the population dynamics of the fungus, given the high heritability of aflatoxin and the relative ease of gaining or losing toxicity via cross-overs and independent assortment, which has significant implications for managing aflatoxin contamination of crops, and for effective biocontrol using atoxigenic A. flavus strains (Olarte et al., 2011). However, 
it should be noted that biological control alone may not be sufficient to completely eliminate the risk of aflatoxin contamination. It must be integrated with other pre- and postharvest crop management strategies including deployment of resistant cultivars (Menkir et al., 2006; Ortiz et al., 2007; Abbas et al., 2009; Hell et al., 2010; Hell and Mutegi, 2011). Furthermore, it is suggested that a network approach involving all stakeholders both from public and private sectors, advanced research institutes, development investors and state or national governments, and active participation by farmers' organizations must be in force to share responsibility for developing crop management strategies that allow farmers produce aflatoxin-free food and feed crops.

Integrated management of aflatoxin provides farmers with natural, safe, and cost-effective solution to prevent aflatoxin contamination of maize and peanut in Africa. This integrated management of aflatoxin uses the biocontrol product "Aflasafe", which is now available for commercialization in Nigeria. Researchers are assessing various strains for further selection of the most effective ones for the development of a biocontrol product in Kenya (http:// r4dreview.org/2011/11/initiative-tackles-killer-aflatoxin/). More such integrated projects are needed to provide effective control of aflatoxin contamination in food crops, especially in the developing countries around the world.

Biocontrol together with the use of resistant cultivars is probably the most cost-effective strategy to reduce the risk of aflatoxin contamination in maize and peanut. However, the cost of biocontrol varies depending on the product and the locale. For example, the cost in the use of AF36 and Afla-Guard ${ }^{\mathrm{TM}}$ to control aflatoxin in cotton and peanut in the USA, respectively, ranges from US\$ 6 to 16 and US\$17-32 acre ${ }^{-1}$, while the cost of biocontrol using local atoxigenic strains of $A$. flavus in Nigerian maize is about US\$10-12 $\mathrm{ha}^{-1}$ (Khlangwiset and Wu, 2010 and references therein).

\subsection{A System-Based Approach to Control Aflatoxin Contamination}

Biosynthesis of mycotoxin is strongly dependent on substrate composition, $\mathrm{pH}$, water activity, temperature or modified environments (Sanchis and Magan, 2004; Ribeiro et al., 2006; Giorni et al., 2008). Combinations of these ecophysiological factors either completely inhibit or fully activate the biosynthesis of aflatoxin. Knowledge of such relationships enables to establish as to which parameter combinations can control aflatoxin biosynthesis, and which are conducive to aflatoxin contamination; and this in the long run is beneficial to develop a system-based approach to predict and control mycotoxin contamination in food and feed crops. 
Schmidt-Heydt and Geisen (2007) developed a mycotoxin biosynthesis genes-specific microarray carrying oligonucleotides of the fumonisin, aflatoxin, ochratoxin, trichothecene and patulin biosynthesis pathways, with in-built option to add any newly identified pathway genes. The initial application of this microarray demonstrates that it is specific in detecting the signals of gene expression under conditions conducive for mycotoxin biosynthesis, with insignificant cross-hybridizations. Recently, Lezar and Barros (2010) reported a diagnostic oligonucleotide microarray to identify the most common food-borne fungi, as well as the genes, leading to toxin production. By using this array, they could identify 32 fungi, and determined their potential to produce mycotoxins. These technological innovations offer opportunity to identify food-born fungi and associated most common mycotoxins, and to investigate the influence of environmental parameters on the activation of the mycotoxin biosynthesis genes and thereby on mycotoxin biosynthesis.

The optimum temperature for the production of aflatoxin is between 28 and $30^{\circ} \mathrm{C}$, and the toxin decreases as temperatures approach $37^{\circ} \mathrm{C}$, which is the optimum temperature for fungal growth. O’Brian et al. (2007) studied the effect of temperature on aflatoxin production and the expression of more than $5000 \mathrm{~A}$. flavus genes at 28 and $37^{\circ} \mathrm{C}$. A total of 144 genes were differentially expressed between the two temperatures, with all aflatoxin biosynthetic genes highly expressed at $28^{\circ} \mathrm{C}$ relative to $37^{\circ} \mathrm{C}$, and the transfer of an aflatoxin-producing culture from $28{ }^{\circ} \mathrm{C}$ to $37^{\circ} \mathrm{C}$ quickly turns of aflatoxin biosynthesis. Schmidt-Heydt et al. (2009) investigated the effects of varying combinations of water activity, $a_{\mathrm{w}}$, and temperature effects on the activation of aflatoxin biosynthesis genes in A.flavus. They found that certain combinations of $a_{\mathrm{w}}$ and temperature, especially combinations that imposed stress on the fungus, resulted in a significant reduction of the growth rate and at these conditions the induction of the whole aflatoxin biosynthesis gene cluster occurred, however, aflatoxin $\mathrm{B}_{1}$ produced was low. In all other combinations $\left(25^{\circ} \mathrm{C} / 0.95\right.$ and $0.99 ; 30{ }^{\circ} \mathrm{C} / 0.95$ and $0.99 ; 35^{\circ} \mathrm{C} / 0.95$ and 0.99) they detected a reduced basal level of cluster gene expression, but a high fungal growth and high aflatoxin production. At single gene comparison, two groups with different expression profiles in relation to $a_{\mathrm{w}}$ and temperature combinations occurred, which were coordinately localized within the aflatoxin gene cluster. The ratio of aflR/aflJ expression correlated with increased aflatoxin biosynthesis. Schmidt-Heydt et al. (2010) further studied the influence of $a_{\mathrm{w}}(0.90-0.99) \times$ temperature $\left(17-42{ }^{\circ} \mathrm{C}\right)$ interactions on growth and the biosynthesis of aflatoxin in A. parasiticus. Optimum growth 
was at $35^{\circ} \mathrm{C}$, but marginal growth occurred at 17 and $42{ }^{\circ} \mathrm{C}$. The optimum conditions for the biosynthesis of aflatoxins $\mathrm{B}_{1}$ and $\mathrm{G}_{1}$ in $A$. parasiticus differed, which did not coincide with the growth optimum $\left(35^{\circ} \mathrm{C}\right)$, but was either below (aflatoxin $\mathrm{G}_{1}, 20-30{ }^{\circ} \mathrm{C}$ ) or above the optimum temperature (aflatoxin $\mathrm{B}_{1}, 37^{\circ} \mathrm{C}$ ). More importantly, aflatoxin $\mathrm{B}_{1}$ synthesis was independent of $a_{\mathrm{w}}$ at certain temperatures as long as $a_{\mathrm{w}}$ was above 0.90 , whereas the optimum production of aflatoxin $G_{1}$ was more dependent on $a_{\mathrm{w}}$ than on temperature in the range $20-30{ }^{\circ} \mathrm{C}$. Such shift between optimum toxin production and optimum fungal growth has also been reported for ochratoxin production by Penicillium verrucosum, trichothecene production by Fusarium culmorum (Schmidt-Hedyt et al., 2008), fumonisin production by Fusarium verticillioides (Jurado et al., 2008) or aflatoxin $\mathrm{B}_{1}$ production by $A$. flavus in peanut (Abdel-Hadi et al., 2010). Furthermore, the expression profiles of two regulatory genes at the different combinations of $a_{\mathrm{w}}$ and temperature showed a relationship with the production profiles of two different aflatoxins produced by $A$. parasiticus. The aflR gene expressed at higher levels at above $30^{\circ} \mathrm{C}$, whereas the most prominent expression of the aflS gene was at below $30{ }^{\circ} \mathrm{C}$, which corresponded with the production profile of either aflatoxin $B_{1}$ or $G_{1}$. Schmidt-Heydt et al. (2009) in an earlier study reported that the ratio between aflS and aflR expression seems an indicator for the activation of aflatoxin $\mathrm{B}_{1}$ biosynthesis in A. flavus.

More recently, Abdel-Hadi et al. (2011) investigated the relative expression of 10 key genes involved in aflatoxin biosynthesis pathway with respect to environmental factors $\left(a_{\mathrm{w}}, 0.995-0.90\right.$; temperature, $\left.20-42{ }^{\circ} \mathrm{C}\right)$ on production of aflatoxin $\mathrm{B}_{1}$ by $A$. flavus. The optimum conditions for the growth of $A$. flavus were $30-35{ }^{\circ} \mathrm{C}$ and $0.99 a_{\mathrm{w}}$, with marginal conditions at 15 and $40{ }^{\circ} \mathrm{C}$ at $0.99 a_{\mathrm{w}}$. For aflatoxin $\mathrm{B}_{1}$ production, the optimum conditions were between 25 and $30{ }^{\circ} \mathrm{C}$ at $0.99 a_{\mathrm{w}}$ and this changed to between 30 and $35^{\circ} \mathrm{C}$ at $0.95 a_{\mathrm{w}}$. They used mixed growth model to relate the relative expression of these genes under different interacting environmental factors to growth and aflatoxin $\mathrm{B}_{1}$ production, and developed a predictive model that gave a good relationship between the observed and predicted aflatoxin $\mathrm{B}_{1}$ production. Schmidt-Heydt et al. (2011) also reported that changes in $a_{\mathrm{w}}$ and temperature significantly affect fungal (Fusarium culmorun and F graminearum) growth and expression of six transcription genes (TRI4, TRI5, TRI6, TRI10, TRI12 and TRI13) from trichothecene gene cluster significantly associated with toxin production, DON, in wheat. These results suggest complex interactions between gene expression, environmental factors and mycotoxin production. This is a powerful tool for understanding the role of genes in relation to 
environmental factors for the development of effective targeted control strategies for mycotoxins. However, an effective integration of molecular, ecophysiological and mycotoxin data are critical in predicting the relative risk of mycotoxin contamination under different stress scenarios that have an impact on both food quality and food security (Magan et al., 2011).

To sum up, it is reasonable to believe that food and feed crops, in years to come due to climate change and variability, will be subjected to more frequent precipitation, drought and high temperature or altered pest-disease scenario, which will result into increased threat to food and feed contamination by mycotoxin-producing fungi. Researchers are now using crop simulation models to predict fungal growth and aflatoxin production. New tools such as GIS, geostatistics and satellite (imagery) data are being employed to monitor shift in community structure of aflatoxin-producing fungi and predict geographic regions with low risk to aflatoxin production. Simple and inexpensive assays such as cELISA or Afla-ELISA are available which are adopted to monitor aflatoxin contamination in crops such as maize and peanut. With the decoding of $A$. flavus genome, it became clear that 25 genes clustered within a 70-kb DNA region in chromosome are involved in the biosynthesis of aflatoxins, of which, the function of 19 genes are known. Several patterns of deletion of aflatoxin genes have been found associated with the loss of aflatoxin-producing ability in some Aspergillus isolates, while others had all the aflatoxin biosynthesis pathway genes, but with defects. With the development of mycotoxin biosynthesis genes-specific microarray, the researchers have been able to study the effects of nutrition, water activity $\left(a_{\mathrm{w}}\right)$, temperature and modified atmosphere and their interactions to predict and identify aflatoxin biosynthesis genes associated with fungal growth and mycotoxin production, which is guiding researchers to adapt a system-based approach to control mycotoxin contamination. Several management options, both pre- and postharvest including the use of resistant cultivars and biocontrol agents, are available to reduce the risk of aflatoxin contamination.

\section{AGROBIODIVERSITY TO ENHANCE NUTRITIONAL QUALITY OF FOOD CROPS}

\subsection{Global Warming Changes Plant and Soil Biodiversity}

\subsubsection{Plant Biodiversity}

Today, 150 plant species (out of 250,000 known plant species) dominate the world's agricultural landscapes, and only 12 crop species provide $80 \%$ of the world's food (Motley et al., 2006). Agricultural biodiversity is crucial 
to coping with climate change as the entire diversity of genes, species and ecosystems in agriculture represents the resource base for food (Kotschi, 2007; Ortiz, 2011). Gene banks around the world, including those of the CGIAR Consortium, maintain about 7.4 million accessions of plant genetic resources, with cereals and legumes constituting $52 \%$ of the accessions (SWPGRFA, 2009). The CGIAR Consortium holds about $3 / 4$ million accessions of 3446 species from 612 genera. In spite of these large collections maintained ex situ, there are still important collection gaps that must be addressed before these priceless genetic resources are lost as a result of climate change or other driving forces leading to the genetic erosion and loss of biodiversity (SWPGRFA, 2009). These ex situ collections are to a large extent safe from the adverse impact of climate change. Unlike cultivated germplasm, there are difficulties associated with ex situ conservation of CWR due to their specific crop husbandry, tendency for natural pod dehiscence, seed shattering and seed dormancy, high variability in flowering and seed production, and rhizomatous nature of some of the species. There is a growing interest that CWR should be preserved in situ in protected areas to ensure evolutionary processes of wild species contributing new variants, which as and when captured by plant explorers, should be able to contribute to addressing new challenges to agricultural production (Ortiz, 2011). Worldwide, there are 76,000 protected areas, spread in $\sim 17$ million $\mathrm{km}^{2}$, and various countries have taken initiatives for establishing CWR in situ conservation (Meilleur and Hodgkin, 2004; Maxted et al., 2008; Maxted and Kell, 2009; SWPGRFA, 2009).

The Intergovernmental Panel on Climate Change (IPCC) predicts that by 2100 the temperature will rise in the range of $1.1-6.4{ }^{\circ} \mathrm{C}$ due to global warming, which will have serious consequences for global agriculture and food production (IPCC, 2007; Lobell et al., 2008). There may be a great loss of biodiversity due to global warming, because organisms are no longer adapted to this changed environment (McLaughlin et al., 2002; Thomas et al., 2004; Biggs et al., 2008). For example, the sub-Saharan Africa and the Caribbean regions are projected to suffer a decline in land area suitable for cultivation of the crops currently grown; while there will be increased opportunities to expand the crop acreage in Europe and North America (Fischer et al., 2002; Lane and Jarvis, 2007). However, the projected gains in area will occur in the regions where these crops are currently not an integral component of food security. This situation may lead to a significant threat to agrobiodiversity, increasing genetic erosion of landraces and threatening wild species, including CWR (Jarvis et al., 2008b). 
Kelly and Goulden (2008) predict that changes in climate could rapidly shift species distribution - some species may expand to newly favorable areas while others may decline in increasingly adverse locations. Using the current and projected future climate data for 2055 and a climate envelope species distribution model, Jarvis et al. (2008a) predicted the impact of climate change on the wild relatives of cowpea (Vigna spp.), peanut (Arachis spp.) and potato (Solanum spp.), which revealed that climate change affect them. An estimated 16-22\% (depending on migration scenario) of these CWR are predicted to go extinct and most species losing over $50 \%$ of their geographic range, and becoming highly fragmented. The CWR of peanut will be the most affected, while those of cowpea may be the least affected. Similarly, today the Western Ghats in southwestern India are very rich in Vigna and Cajanus species. However, with changes in temperature and photoperiod coupled with other factors such as habitat destruction, their CWR populations are becoming alarmingly less, which calls for a strategy for collecting and conserving CWR in this region of India (Upadhyaya et al., 2011e).

Global warming is also impacting changes in plant phenology. For example, some species show advancement in timing of phenological events such as breeding or flowering, of 2.3 days per decade (Parmesan and Yohe, 2003), while others exhibit an evolution of multiple life-history traits (Franks and Weis, 2008), new variants for phenological traits (Vigouroux et al., 2011), or shrinking body sizes (Sheridan and Bickford, 2011). All these events could negatively impact both crop plants and protein sources, which are important to human nutrition (Sheridan and Bickford, 2011).

Franks and Weis (2008) investigated the effects of 5 years of prolonged drought on life-history traits in an annual plant Brassica rapa. By comparing the ancestors with their descendants, they found that drought caused many changes in life-history traits, including a shift to earlier flowering, reduced peak flowering, and greater skew of the flowering schedule. Descendants had thinner stems and fewer leaf nodes at the time of flowering than ancestors, indicating that the drought selected for plants that flowered at a smaller size and earlier ontogenetic stage rather than selecting for plants to develop more rapidly. The Sahel will be the most adversely affected in sub-Saharan Africa due to extreme variation in climate change as a result of frequent droughts of varying intensity and duration. Pearl millet in Niger is the main staple crop, and farmers grow this crop in $65 \%$ of the cultivated area for producing more than $80 \%$ of the entire caloric intake in the country. Vigouroux et al. (2011) analyzed the impact of drought on variation in phenological and morphological differences in pearl millet landraces collected 
from the same villages in Niger in 1976 and 2003. Their study revealed no major changes in the main cultivars or in their genetic diversity. However, these authors observed a significant shift in the adaptive traits. Samples collected in 2003 displayed a shorter life cycle, increased frequency of early flowering allele at the PHYC locus, and a reduction in plant and spike size in comparison to the sample collected in 1973. This clearly indicates that recurring drought can lead to selection of earlier flowering in major crops in the Sahelian region.

The increased $\mathrm{CO}_{2}$ and $\mathrm{N}$ supply often drive plant stoichiometry in opposite directions (Ainsworth and Long, 2005; Novotny et al., 2007), but the productivity in the same direction (Ainsworth and Long, 2005; Luo et al., 2006). Several studies have shown that increases in $N$ availability decrease plant diversity (Stevens et al., 2004; Suding et al., 2005; Harpole and Tilman, 2007; Clark and Tilman, 2008; Hautier et al., 2009). Likewise, elevated $\mathrm{CO}_{2}$ (hereafter $\mathrm{eCO}_{2}$ ) reduces plant diversity (Oren et al., 2001; Ainsworth and Long, 2005; Reich et al., 2006a,b). However, no consensus has emerged so far as to how rising $\mathrm{CO}_{2}$ concentrations interact with $\mathrm{N}$ to influence plant biodiversity (Zavaleta et al., 2003; Niklaus and Kröner, 2004). In a long-term (10 years) open-air experiment involving 16 grassland species grown under all combinations of ambient $\mathrm{CO}_{2}\left(\mathrm{aCO}_{2}\right)$ and $\mathrm{eCO}_{2}$ and ambient $\mathrm{N}(\mathrm{aN})$ and elevated $\mathrm{N}(\mathrm{eN})$, Reich (2009) found that eN reduces species richness by $16 \%$ at $\mathrm{aCO}_{2}$ but by just $8 \%$ at $\mathrm{eCO}_{2}$. This resulted from multiple effects of $\mathrm{CO}_{2}$ and $\mathrm{N}$ on plant traits and the soil that altered competitive interactions among species. Hence, $\mathrm{eCO}_{2}$ ameliorated the negative effects of $\mathrm{N}$ enrichment on species richness.

\subsubsection{Soil Biodiversity}

Soil biodiversity exceeds the aboveground systems biodiversity, and is crucial for the sustainability of agroecosystems (Wardle, 2002). It consists of macrofauna or soil engineers (earthworms and termites), mesofauna (microarthropods such as mites and springtails), microfauna (nematodes and protozoans), and microflora (bacteria and fungi). The soil organisms perform a number of vital functions such as decomposition and degradation of plant litter and cycling of nutrients; converting atmospheric nitrogen into organic forms (immobilization) and remineralization of mineral nitrogen, leading to the formation of gaseous nitrogen; suppression of soil pathogens through antagonism; regulating microclimate and local hydrological processes; synthesizing enzymes, vitamins, hormones, vital chelators and allelochemicals that regulate population and processes; and altering soil structure and other soil 
physical, chemical and biological characteristics (Paoletti et al., 1994; Altieri, 1999). In addition to the effects on soil desertification, land use pattern and soil pollution-including those resulting from $\mathrm{N}$ enrichment-alter soil biodiversity (Wall et al., 2010; Sylvain and Wall, 2011; Prichard, 2011). The changes in soil biodiversity are observed through effects on soil organisms as a result of the changes in temperature and precipitation and through climate-driven changes (rising atmospheric $\mathrm{CO}_{2}$ and warming) in plant productivity and species composition, as discussed in the following.

Accumulated evidence so far reveals that soil biota is vulnerable to global changes and soil disturbance. A metaanalysis—involving data from over 75 experiments-revealed that soil biota response to global change are predictable and unique for each global change factor. For example, positive-effect size of $\mathrm{eCO}_{2}$ on abundance of soil biota diminished with time, whereas the negative-effect size of warming and positive-effect size of precipitation intensified with time. Likewise, the abundance of microflora and microfauna increased with $\mathrm{eCO}_{2}$. The effects of $\mathrm{CO}_{2}$ varied between field and greenhouse studies, less in the former while more pronounced in the latter (Blankinship et al., 2011 and references therein). In a 5-year open-top chamber experiment involving single and combined effects of $\mathrm{aCO}_{2}$ and $\mathrm{eCO}_{2}$, ambient and elevated temperature and changes in precipitation, Kardol et al. (2011) concluded that changes in soil moisture content, either as a direct effect of the changes in precipitation or as an indirect effect of warming or $\mathrm{eCO}_{2}$, had a larger impact on microarthropods communities than did the direct effects of warming and $\mathrm{eCO}_{2}$. In a multifactor climate change experiment, Castro et al. (2009) reported increased fungal abundance in warmed treatments, increased bacterial abundance in warmed plots with $\mathrm{eCO}_{2}$ but decreased in warmed plots under $\mathrm{aCO}_{2}$, changes in precipitation altered the relative abundance of proteobacteria and acidobacteria where acidobacteria decreased with a concomitant increase in the proteobacteria in wet relative to dry treatments, altered fungal community composition due to the changes in precipitation, and differences in relative abundance of bacterial and fungal clones varied among treatments. All these observations led the authors to conclude that climate change drivers and their interactions among them may cause changes in the bacterial and fungal abundance, with precipitation having greater effect on the community composition.

Dominique et al. (2006) exposed different sets (5, 12, or 31) of plant species (grasses) to $\mathrm{aCO}_{2}$ or $\mathrm{eCO}_{2}$ for 5 years to analyze how plant diversity affects below ground diversity. The results revealed that increased levels of $\mathrm{CO}_{2}$ had no significant influence on both soil bacterial community 
composition and bacterial richness. In contrast, the variability in plant diversity level had significant effects on bacterial composition but no influence on bacterial richness. This research therefore suggests that the soil microbial composition is mainly related to plant diversity, assuming that different plant species might harbor specific rhizospheric microbial populations, rather than altered soil carbon fluxes induced by $\mathrm{eCO}_{2}$ that lead to increased photosynthesis. Bardgett (2011) points out that there is sufficient evidence to show that the transfer of carbon through plant roots to the soil plays a primary role in regulating ecosystem responses to climate change and its mitigation. Research to answer this question, however, continues, e.g. what is the mechanism involved in transfer of plant carbon to soil, its consequences for ecosystem carbon cycling, and the potential to exploit plant-root traits and soil microbial processes that favor soil carbon sequestration are issues that need further research?

Very little is known about the influence of $\mathrm{eCO}_{2}$ on the structure and functioning of below ground microbial community. In a 10-year field exposure of a grassland ecosystem to eCO2, Zhili et al. (2010) detected dramatic alteration in the structure and functional properties of soil microbial communities. They found that the total microbial and bacterial biomass significantly increased under $\mathrm{eCO}_{2}$, while the fungal biomass remained unaffected. Furthermore, the structure of microbial communities was markedly different between $\mathrm{aCO}_{2}$ and $\mathrm{eCO}_{2}$. More recently, using tag-encoded pyrosequencing of $16 \mathrm{~S}$ rRNA genes, Deng et al. (2012) also found that the soil microbial community composition and structure were significantly altered under $\mathrm{eCO}_{2}$. In both the studies, the changes in microbial structure was significantly correlated to soil moisture, soil status relative to $\mathrm{C}$ and $\mathrm{N}$ contents, and plant productivity.

\subsection{High-Throughput Assays for Monitoring Nutritional Traits}

There is greater emphasis in plant-breeding programs worldwide to select and or develop cultivars that are high yielding as well as their grains or stover are also more nutritious to meet both total calorie and nutritional demands of the growing populations, and for improving livestock productivity. However, one essential requirement for progress in this area of research is the timely and economic determination of quality traits of a large number of samples, as often required in breeding programs, which is laborious, time consuming, and expensive. Obviously, there is a need to develop analytical assays that are nondestructive, high throughput, fast, accurate, cost-effective, requiring relatively small samples, and allowing a simultaneous profiling of multinutrient elements. Assays 
based on the use of wet chemistry are the most accepted to measure the levels of seed components. However, they require a relatively large sample size, and are destructive, time consuming, and relatively slow when a large numbers of samples need to be screened in a short period to allow breeders make a decision about the breeding lines for generation advance in the following crop season.

\subsubsection{Minerals from the Soil Samples}

Numerous methods have been developed for extracting fractions of plant nutrients, which are available to plants in the field. A universal problem with all these methods is relative to the selection of a ubiquitous extractant due to the fact that these extractants suffer from shortcomings to accurately mimic the field conditions during the growing season, which generally leads to poor prediction, accuracy and lack of robustness (Sinclair and Edwards, 2008 and references therein). Salts of Ethylenediamine tetra acitic acid (EDTA) and Diethylenetriamine pentaacetic acid (DTPA) are the most commonly used for predicting the availability of micronutrients to the plants in the field (Sahrawat et al., 2002, 2011; Sinclair and Edwards, 2008).

The technique diffusive gradient in thin-films (DGT) measures the diffusive supply of elements, thereby mimicking plant roots' role in accessing and uptake of nutrient elements. This technique differs from the other extraction techniques by responding to kinetics of release from soil rather than pseudoequilibrium between the extractant and soil nutrients. It was earlier used to access bioavailable trace elements in waters, heavy metals in contaminated soils and P in waters and soils (Zhang et al., 1998, 2001; Menzies et al., 2005; McBeath et al., 2007). Tandy et al. (2011) tested the ability of DGT technique to assess plant-available $\mathrm{P}, \mathrm{Zn}$ and $\mathrm{Cu}$ in a wide range of Scandinavian soils and compared the results with those obtained using conventional laboratory methods (EDTA) and DTPA for $\mathrm{Cu}$ and $\mathrm{Zn} ; \mathrm{NaHCO}_{3}$ for $\mathrm{P}$ and soil solution concentrations). The results of the study showed that the soil test values obtained by the DGT method better predicted the concentrations of various nutrients in the youngest fully developed leaf of pot-grown barley plants. They also reported that the DGT method could predict accurately plant uptake of $\mathrm{Zn}$ and $\mathrm{P}$, while the conventional soil test methods based on the extraction of nutrients using chemical extractants performed poorly. The results with the DGT technique were also more accurate in predicting $\mathrm{Cu}$ concentration in the leaves. Thus, Tandy et al. (2011) concluded that the DGT-based method is more accurate at predicting plant-available $\mathrm{P}, \mathrm{Zn}$ and $\mathrm{Cu}$ than commonly used methods for analyzing plant-available nutrients in soils. However, while analyzing a large 
number of samples on a routine basis, simplicity, rapidity and cost involved need to be considered in addition to of course the precision in the analyses of samples (Sahrawat et al., 2011; Sahrawat and Wani, 2013).

\subsubsection{Minerals from Plant Tissues or Grains Samples}

Inductively coupled plasma-mass spectroscopy (ICP-MS) is the state-of-theart technique used for plant analysis for multinutrients with a high sensitivity. However, fast and thorough sample digestion of plant materials is a time-consuming task, and is a major bottleneck in modern multielemental analysis. Additional limitation may be the sample size or too low concentration of elements in the sample. Hansen et al. (2009) developed a highthroughput microscaled method that enables digestion of small quantity of plant sample for elemental profiling and distribution of trace elements between and within plant organs by ICP-MS. In comparison to existing vial-in-vial systems, it represents a significant methodological advancement in terms of higher capacity, reduced labor and material costs, less contamination and improved accuracy following the introduction of microscaled digestion of plant samples. Furthermore, the results from this method have also shown to be in good correspondence with the single grain concentrations and those obtained by analysis of bulked samples of milled rice grain (Hansen et al., 2009). Thus, the use of single grains for analysis of elements in cereals provides a valuable tool for high-throughput screening of a large number of lines in plant-breeding programs. More recently, Husted et al. (2011) have developed a novel hyphenated technique, which is based on liquid chromatography and ICP-MS (LC-ICP-MS) and has been extensively complemented by molecular mass spectrometry for structural information of biologically relevant plant species. Moreover, Wheal et al. (2011) reported another novel closed-tube nitric acid/hydrogen peroxide digestion method for inductively coupled plasma optical emission spectrometry (ICP-OES) for analysis of plant tissue, which they tested on six botanical reference materials. This method they found superior over to open-tube digestion of reference materials, as it showed less values of blanks as a result of probably less contamination than that in the case of open-tube digestion method. The closedtube method is as efficient as the open-tube digestion of reference materials recorded $94-113 \%$ recovery of published concentrations for most essential elements in comparison to $93-115 \%$ recovery by the open-tube digestion method, with recovery standard deviation comparable in the two methods (2-6\% for the closed-tube and 1-8\% for the open-tube method). Because of its fixed digest time $(2.5 \mathrm{~h})$, it is short enough to conduct three digest runs per 
working day per digestion block, while in the open-tube digestion method, it takes relatively longer time for the digestion of plant materials, especially with dense materials, reducing the daily throughput considerably.

Near infrared (NIR) reflectance-a promising technique discovered by Friedrich Herschel in 1800 (Davies, 2000)-has found wide application in estimating various chemical components in seed, plants and food in many crops. It covers the range of the electromagnetic spectrum from 780 to $2500 \mathrm{~nm}$. Essentially, the product (including the seed) is exposed to NIR irradiation, and the reflected or transmitted radiation is measured. While the radiation penetrates the product, its spectral characteristics change through wavelength-dependent scattering and absorption. This change depends on the chemical composition of the product, as well as on its light scattering properties, which are related to the plant microstructure. Multivariate statistical techniques such as partial least squares regression are then applied to extract the required information from the usually convoluted spectra (Lee et al., 2011). The NIR technology has been applied to predict oil per se and its quality in rapeseed, safflower, and soybean (Tajuddin et al., 2002; Kim et al., 2007; Patil et al., 2010; Rudolphi et al., 2012; Wittkop et al., 2012); fiber fractions in rapeseed (Wittkop et al., 2012); starch, protein, oil, amino acid composition and weight of individual grains in maize (Spielbauer et al., 2009; Tallada et al., 2009; Rosales et al., 2011); stover quality in maize (Melchinger et al., 1986); amino acid composition in soybean (Kovalenko et al., 2006); protein, starch and seed weight in intact seed in common bean (Hacisalihoglu et al., 2010); and for sensing moisture content of in-shell peanut (Sundaram et al., 2012).

Baianu et al. (2012) developed a high-resolution nuclear magnetic resonance and NIR models that they validated by estimating amino acid profiles of proteins from a large number of single and bulked soybean seeds, without protein extraction from the seed. Using partial least squares regression technique to analyze the NIR spectral data, they found that single soybean seed NIR spectra are broadly similar to those of bulk whole soybeans, with the exception of minor peaks, $950-1000 \mathrm{~nm}$, in single soybean NIR spectra. The highly resolved NIR chemical images that they obtained for selected regions of mature soybean embryos allowed for the quantitation of oil and protein components, extending the NIR sensitivity range to the picogram level, with submicron spatial resolution in the component distribution throughout intact soybean seeds and embryos. These technological innovations are potentially important for the application of biotechnology that requires rapid and ultrasensitive analyses, such as those concerned with high-content microarrays in genomics and proteomics research. 
Several spectroscopy methods are now available for studying the accumulation and distribution of essential nutrients in the seed. Proton-induced $\mathrm{X}$-ray emission targets the embryo region. Scanning and transmission electron microscopy (STEM) in combination with energy-dispersive X-ray (EDX) microanalysis, or STEM-EDX, focuses on aleurone and scutellum cells for providing subcellular information. Nano-secondary ion mass spectrometry visualizes the subcellular distribution but limited to regions of only a few square micrometers. X-ray fluorescence $(\mu-\mathrm{XRF})$ provides elemental maps for various elements in whole grain sections. Maia captures intricate detail in natural material. These assays differ in terms of resolution and sensitivity, depth of analysis, and in their capacity to provide mass resolution or molecular information (Dwivedi et al., 2012 and references therein). Paltridge et al. (2012) investigated energy-dispersive X-ray fluorescence spectrometry (EDXRF) for measurement of $\mathrm{Zn}$ and $\mathrm{Fe}$ in whole grains of rice and pearl millet. The results obtained by EDXRF were variable, but highly correlated $\left(r^{2}=0.79-0.98\right)$ with those determined using ICP-OES values for both $\mathrm{Zn}$ and Fe in both the species, with predicted values for $\mathrm{Zn}$ and $\mathrm{Fe}$ in rice to within 1.9 and $1.6 \mathrm{mg} \mathrm{kg}^{-1}$ of ICP-OES values, and for $\mathrm{Zn}$ and $\mathrm{Fe}$ in pearl millet to within 7.6 and $12.5 \mathrm{mg} \mathrm{kg}^{-1}$ of ICP-OES values, at $95 \%$ confidence levels. Hence, this assay offers a convenient, economical tool for screening a large number of samples for $\mathrm{Zn}$ and Fe in rice and pearl millet, which can also be used on grains of other species, or for other mineral elements, with some modification including crop-specific calibrations.

Lorenz et al. (2007) developed a rapid and an inexpensive method for measuring phytate and inorganic phosphorus ( $\mathrm{Pi}$ ) concentrations in maize, which provides adequate precision and simplicity to deal with a large number of breeder's samples for estimating phytate and Pi levels simultaneously. Estimates obtained from this technique match closely with those obtained from ion exchange methods, and the repeatability of the values across fields suggests that the protocol can be used to make heritable measurements for both phytate and Pi.

Hulshof et al. (2007) developed a fast screening method for estimating $\beta$-carotene in maize seeds. This method is based on the principle of semiquantitative analysis that allows distinction between lines-without the need of a full HPLC analysis (Rodriguez-Amaya and Kimura, 2004) - the low, medium and high levels of provitamin A carotenoids, thereby reducing analysis costs. It is an appropriate assay to initially discard low $\beta$-carotene lines; and to carry out the HPLC analysis for $\beta$-carotene with select group of lines. 


\subsection{Profiling Genetic Variation for Nutritional Traits}

Genes for desirable traits are embedded in biodiversity. The genetic variation in crops genepool including wild relatives, when systematically characterized, evaluated and documented, and dissected through applied genomic tools provides crop genetic enhancers for the agronomically important gene(s) and their allelic forms to develop crops cultivars that are more productive and nutritious. For example, a key regulating gene that regulate oil content and oil composition in maize, a gene responsible for increasing the flux of $\beta$-carotene in maize, or a wild-species allele (Brix9-2-5) for increasing the sugar yield of tomato (Lippman et al. 2007; Zheng et al., 2008; Harjes et al., 2008). These few examples demonstrate the usefulness of agrobiodiversity (both cultivated germplasm including landraces and wild and weedy relatives) to discover new sources of variation and to mine alleles associated with such variation for improving the quality of food crops (Zamir, 2008). In recent years, there has been increased emphasis to enhance the nutritional value of the food crops that constitute the bulk of the calories, particularly in the developing world (Dwivedi et al., 2012 and references cited therein). The CGIAR Consortium gene bank holdings include about 0.422 million accessions of cereals and legumes genetic resources (www.singer.cgiar.org, assessed on 17th April 2012), and in all these crops, the reduced subsets in the form of core (Frankel, 1984) or mini core (Upadhyaya and Ortiz, 2001) collections, representing diversity in the entire collection of a given species, are available (Dwivedi et al., 2005, 2007a and references therein) for use in crops improvement programs. Research to-date suggests that these subsets are ideal resource to find new sources of variation and mine alleles associated with agronomically beneficial traits (Upadhyaya et al., 2009). The CGIAR Consortium gene bank holdings have been extensively characterized for morphoagronomic traits including resistance to abiotic and biotic stresses, whereas very limited information on these genetic resources is available and documented about their chemical attributes (SWPGRFA, 2009). Hence, the need to screen these reduced subsets for seed chemistry to identify germplasm with improved seed composition, for use in breeding programs.

\subsubsection{Variation for Fe, Zn, Phytate and Carotenoids}

The CGIAR Challenge Program HarvestPlus (www.harvestplus.org) provided opportunity to researchers to screen these genetic resources for grain iron $(\mathrm{Fe})$ and zinc $(\mathrm{Zn})$, the two most important micronutrients, and $\beta$-carotene, the precursor of vitamin A (Pfeiffer and McClafferty, 
2007; Bouis and Welch, 2010). The evidence accumulated so far suggests substantial genetic variation for $\mathrm{Fe}$ and $\mathrm{Zn}$ in the grains of both cereals and legumes, e.g. high $\mathrm{Fe}$ in common bean from Chile, Colombia, Peru, Rwanda and Tanzania; high $\mathrm{Fe}$ and $\mathrm{Zn}$ in maize from southern Africa; high $\mathrm{Fe}$ and $\mathrm{Zn}$ in pearl millet from West Africa; high Fe in sorghum from Benin; traditional rice cultivars from India having more $\mathrm{Fe}$ and $\mathrm{Zn}$; and high $\mathrm{Fe}$ and $\mathrm{Zn}$ in finger millet and foxtail millet from China and India (Upadhyaya et al., 2011a,b; Dwivedi et al., 2012 and references therein). Few studies involving core or mini core collections have led to identifying accessions with high grain $\mathrm{Fe}$ and $\mathrm{Zn}$ in common bean, finger millet, foxtail millet and sorghum (Islam et al., 2002; Kumar et al., 2009; Upadhyaya et al., 2011a, b). The CGIAR Consortium continues evaluating chickpea, peanut, pigeonpea, pearl millet, and sorghum mini core collections for variation in grain $\mathrm{Fe}$ and $\mathrm{Zn}$ concentrations; and the preliminary evidence from these ongoing evaluations suggests substantial variation for these traits (H. D. Upadhyaya, unpub.), which clearly suggest the usefulness of the core and mini core subsets for identifying variation for grain-quality traits as well.

CWR have been the source of resistance to many pests and diseases both in cereals and legumes (Dwivedi et al., 2008). Likewise, researchers detected abundant genetic variation in the grain for Fe and $\mathrm{Zn}$ among wild and weedy relatives in common bean and wheat (Guzmán-Maldonado et al., 2000; Chhnneja et al., 2006; Acosta-Gallegos et al., 2007; Xie and Nevo, 2008). Some introgression progenies showed exceptionally high Fe or $\mathrm{Zn}$ in the grain than cultivars of common bean and wheat (Acosta-Gallegos et al., 2007; Neelam et al., 2010; Tiwari et al., 2010).

Phytic acid is the major form of phosphorus storage in the grains. However, its high concentration limits micronutrients bioavailability as it binds with these minerals (Fe and $\mathrm{Zn}$ ) to form mixed salts (phytin), largely excreted by humans and nonruminant animals, with a potential to significantly impact water pollution (Lott et al., 2000). However, phytic acid is vital for seed development, seedling growth and development and may have a positive role as antioxidant and anticancer agent (Oatway et al., 2001). Research to-date suggests availability of low-phytic acid germplasm or mutants in barley, common bean, lentil, maize, rice, sorghum, soybean and wheat (Dwivedi et al., 2012 and references therein) for use in crops breeding. Bioavailability of micronutrients, particularly Fe and Zn, merits research about the usefulness of mineral-dense cultivars in human nutrition. Limited studies in common bean, maize and wheat have shown large differences in Fe and Zn bioavailability, with a few mineral-dense germplasm 
having increased amount of bioavailable Fe/Zn (Dwivedi et al., 2012 and references therein).

Some maize germplasm could be used as source of pro-vitamin A, $\beta$-carotene, $\alpha$-carotene, and $\beta$-cryptoxanthin and the non-provitamin $\mathrm{A}$ including lutein and zexanthin. The metabolite profiling of germplasm collection identified a subset of 10 genetically diverse germplasm representing biochemical extremes for maize kernel carotenoids (Chander et al., 2008a). Hydroxylase 3 alleles contributed to $78 \%$ of the variation and approximately 11 -fold differences in $\beta$-carotene relative to $\beta$-cryptoxanthin and $36 \%$ of the variation and fourfold difference in absolute levels of $\beta$-carotene (Harjes et al., 2008). More importantly, the discovery of a rare genetic variation increases $\beta$-carotene substantially in maize (Yan et al., 2010). Likewise, some of the elite maize inbreds representing major heterotic groups in China were reported to contain high protein (up to $14.7 \%$ ), starch (up to $70.2 \%$ ), oil (up to $5.2 \%$ ), $\alpha$-tocopherol (up to $74.8 \mu \mathrm{g}^{-1}$ ), $\gamma$-tocopherol (up to $78.0 \mu \mathrm{g}^{-1}$ ), $\delta$-tocopherol (up to $5.3 \mu^{-1}$ ) and total tocopherols $\left(120.7 \mu \mathrm{g}^{-1}\right)$, and carotenoids (Chander et al., 2008b). Sorghum landraces, particularly those with yellow endosperm from Niger and Nigeria, have shown significant variation for carotenoids, with lutein, zeaxanthin and $\beta$-carotene, the predominant carotenoids (Salas Fernandez et al., 2008, 2009). Natural variations for

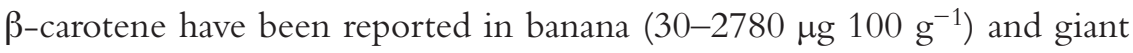
swamp taro (Cyrtosperma chamissonis) (50-2040 $\mu \mathrm{g} 100 \mathrm{~g}^{-1}$ ) (Englberger et al., 2003), cassava (up to $4 \mathrm{mg} \mathrm{kg}^{-1}$ in the cassava landrace UnB 400 from Brazil) (Nassar et al., 2009), and sweet potato (up to $226 \mu \mathrm{g}$ fruit weight ${ }^{-1}$ in a breeding line 11-20 from sweet potato breeding program at North Carolina State University) (Teow et al., 2007).

\subsubsection{Variation for Protein and Oil Concentrations and Their Quality in Maize}

The maize grain protein is deficient in lysine and tryptophan. The discovery in Peru's maize of two mutant alleles, opaque 2 (o2) and floury 2 (fl2), which alter amino acid profile and composition of maize endosperm protein, opened up exciting opportunities to improve maize endosperm protein quality (Mertz et al., 1964; Nelson et al., 1965). However, the mutants adversely affected agronomic performance, and had soft and chalky kernels, not liked by maize growers in developing countries. The researchers used $o 2$ and genetic modifiers (Paez et al., 1969) to remove these defects, and developed a number of agronomically superior maize germplasm or hybrids with better protein quality profile, branded as "quality protein maize", and 
many version of this are currently grown by farmers in sub-Saharan Africa, Brazil, China and South Africa (Vasal, 1999; Krivanek et al., 2007). Another pioneering work in maize relates to Illinois long-term selection (100 cycles of selection) experiment that developed populations exhibiting phenotypic extremes for grain composition and correlated traits. The selection responses of both protein and oil are greater than 20 standard deviations from the original population mean (Dudley, 2008) in the positive direction and four standard deviations in the negative direction. These lines at the present time are being used as a source of favorable alleles associated with oil, protein and starch accumulation (Moose et al., 2004).

\subsubsection{Variation for Improving Oil Quality in Peanut}

Peanut oil quality is determined by the ratio of oleic (O) and linoleic (L) fatty acids. A higher ratio results in a better storage quality of oil, and longer shelf-life of the products (Branch et al. 1990). Norden et al. (1987) were the first to report exceptionally high oleic trait, O/L ratio (40), in the Florida breeding line, F 435. A few elite germplasm with high $\mathrm{O} / \mathrm{L}$ ratio were registered and released as high-oleic $(\mathrm{O} / \mathrm{L}$ ratio $>10)$ peanut cultivars (Olin, SunOleic 95R, SunOleic 97R, and Tamrun OL11) in USA from this source (Gorbet and Knauft, 1997, 2000; Simpson et al., 2003b; Baring et al., 2012). However, F 435 is not easily available to peanut researchers. Recently Upadhyaya et al. (2011c) evaluated a peanut mini core collection (Upadhyaya et al., 2002) for variation in fatty acid profiles, and identified ICG 2381-a subsp. hypogaea accession from Brazil-with O/L ratio $\sim 7$, which is 2-3 times more than normal range reported in cultivated peanut germplasm. It provides a unique source for peanut breeders to improve oil quality of new cultivars.

\subsection{Sustaining Food Quality by Manipulating Soil Microbial Diversity}

The imbalance in demand and supply of food is mainly the result of inherent low soil fertility and the degradation of the natural resources, such as soil and water due to unsustainable farming practices without adequate investment. Such practices lead to the depletion of groundwater, soil organic matter and plant nutrient reserves of not only major but also secondary and micronutrients (Sanchez, 2002; Kijne, 2004; Kibblewhite et al., 2008; Lal, 2009; St. Clair and Lynch, 2010; Sahrawat et al., 2011; Graham et al., 2012). Equally important is that the nutrient-impoverished soils contribute to human malnutrition at least in two ways. First, by reducing crop yields, 
thereby causing food scarcity that results in protein-energy malnutrition. Second, food produced on nutrient-deficient soils contain low concentrations of minerals in plant tissue including critical micronutrients such as $\mathrm{Fe}$ and Zn (Welch, 2002; St. Clair and Lynch, 2010; Sahrawat et al., 2011).

The deficiency of vitamin A, widely deficient in humans and the cause of anemia (Bloem et al., 1989; Graham et al., 2012) is however, not a nutrient for plants; and plants biosynthesize the carotenes that human body converts into vitamin A. Thus, for vitamin A deficiency, there is no fertilizer or nutrient management strategy. However, a viable strategy to provide vita$\min \mathrm{A}$ in food is to introduce carotene-rich secondary staples as supplement in the food system (Welch, 2002; Graham et al., 2012) unlike for nutrients such as iron and zinc, which is based in exploiting the synergy between biofortification and nutrient management via enhanced crop husbandry (Graham et al., 2012).

The evidence suggests that soils deficient in multinutrients including major and micronutrients result in poor grain and fodder quality; and balanced nutrient management not only increases food production but also enhances crop quality relative to protein, oil, and Fe and Zn (Sahrawat et al., 2011 and references therein). The mineral composition of plant foods, which depends on soil nutrients status, has direct implications for human health (Welch, 2002). To enhance crop productivity and quality of food, there is need to simultaneously overcome the constraints posed by low soil fertility, and also addresses the limitations imposed by the impact of climate change on food production and quality through soil fertility improvement in general and nutrient acquisition and utilization by crops in particular (Gavito et al., 2001; Taub et al., 2008).

Microbes are the unseen majority in the soil; and form a large portion of life's genetic diversity. Soil microbes play key roles in agroecosystem; for example, heterotrophs decompose organic matter applied to the soil and release nutrients such as nitrogen and phosphorus in the mineral available form for acquisition by plants, which eventually impact food production and food quality (van der Heijden et al., 2008; St. Clair and Lynch, 2010). Although of great importance, the effects of climate change via soil fertility and microbial diversity on nutrient acquisition and utilization by crops are poorly understood and have not received the attention they deserve (Lynch and St. Clair, 2004). Indeed, soil biota and their diversity are the main drivers of mineral nutrient cycling (Kibblewhite et al., 2008), and play equally important role in causing and suppressing pathogenesis in plants and foods (Garbeva et al., 2004). The microbial activity in the soil affects 
both biotic and biological soil properties that influence plant growth, food production and quality (Bardgett et al., 2005; Barrios, 2007; Cortois and De Deyn, 2012). For example, altering the rhizosphere microflora by seed or root inoculation with specific organisms (i.e. biofertilizers) has long been recognized as a practical strategy with potential to promote plant growth by enhancing the availability of nutrients such as phosphorus or the release of growth promoting substances. There is a large body of literature on the effects of various bacterial cultures on plant growth of a range of crops including both cereals and legumes under controlled conditions, their role in practical agriculture needs to be established by future research (Zahir et al., 2004 and references therein). Nevertheless, the general thinking is that the climate change effects via rising temperature, drought and increase in intense rainfall events are most likely to influence nutrient availability in the soil, and their subsequent acquisition and utilization by crop plants (Sinclair, 1992; Lynch and St. Clair, 2004; St. Clair and Lynch, 2010).

To sustain a positive response to increased $\mathrm{CO}_{2}$ concentration requires an increase in plant uptake of the total amount of plant nutrients, otherwise the crop quality in terms of mineral composition is likely to decline (Gavito et al., 2001; Erbs et al., 2010). However, it is very difficult to predict the response to climate change on nutrient acquisition and utilization by crop plants because of the large uncertainty about the availability of nutrients in the soils as a result of climate change (Sinclair, 1992). For example, while increased $\mathrm{CO}_{2}$ concentration would stimulate biological nitrogen fixation by free-living organisms and symbiotic systems (Sinclair, 1992), whereas increased temperature and altered rainfall pattern and intensity may result in enhanced losses of minerals by leaching and soil erosion (Meadows, 2003; Tang et al., 2008; Zougmore et al., 2009). Such a situation may adversely affect the amount of minerals or nutrients available in the soil for acquisition and utilization by crops. Furthermore, the acquisition, uptake and utilization of minerals by crop plants is further affected by drought, exacerbated by climate change, through the availability and transport of both mass flow and diffusion of plant nutrients in the soil (Barber, 1995; Bassirirad, 2000; Biggs et al., 2008; Brouder and Volenec, 2008). For example, the reduction of root growth, alteration in root architecture and impairment in root functions under drought reduce the mineral acquisition capacity of plant root system (Mackay and Barber, 1985; Barber, 1995). In addition to the effects of drought on nutrient availability, accessibility and acquisition by roots, drought also adversely influences root-microbe associations, which are the principal strategy for mineral capture and acquisition by crop plants. 
For example, it has been observed that due to reduced carbon and oxygen fluxes and nitrogen accumulation in root nodules retard or at times inhibit biological nitrogen fixation in leguminous plants (Dakora and Keya, 1997; González et al., 2001; Ladrera et al., 2007). Equally importantly, drought also alters both composition and activity of soil microbial community that control carbon and plant nutrient transformations, availability and cycling (Schimel et al., 2007; St. Clair and Lynch, 2010).

The soil microbial organisms are the foundation of soil fertility maintenance and efficient plant nutrition in food production systems. Moreover, the widespread presence of the Arbuscular mycorrhiza (AM) symbiosis in nodulated legumes and the role of AM fungi in improving nodulation and biological nitrogen fixation are universally recognized.The synergy between biological nitrogen fixation and AM fungi leads to improved availability and accessibility of phosphorus and mitigation of water stress by the action of AM fungi. At times, the AM fungi may also be involved in the biological control of root pathogens. The role of the AM fungi in an overall improvement of soil quality is yet another factor involved (Barea et al., 2005).

Studies have shown that the root-mycorrhizal symbiosis in plants is not very sensitive to soil moisture stress of moderate intensity (Entry et al., 2002; Garcia et al., 2008; St. Clair and Lynch, 2010). In fact, there is a large body of literature documenting the beneficial effects of mycorrhizal fungi in plants under varying degrees of drought or soil moisture stress conditions (Entry et al., 2002; Jones et al., 2004; Garcia et al., 2008); and indeed a part of the benefit provided by mycorrhizae under drought stress is associated with increases in mineral nutrient transfer to plant roots (Goicoechea et al., 1997; Jones et al., 2004; St. Clair and Lynch, 2010; Cavagnaro, 2008; Cavagnaro et al., 2010).

The information on the effects of soil warming suggest that nutrient uptake by crop plants could increase from $100 \%$ to $300 \%$ by enlarging the root surface area that will promote increased rates of mineral diffusion and water influx (Ching and Barber, 1979; Mackay and Barber, 1985). Likewise, warm temperature increases rates of transpiration and as a consequence plants tend to acquire water-soluble nutrients at enhanced rates as temperature increases (Barber, 1995). In addition, temperature increase can also stimulate nutrient acquisition by plants via both faster ion diffusion rates and increased root metabolism (Bassirirad, 2000). However, such positive effects of warm temperature on mineral acquisition by plants are dependent on the availability of adequate soil moisture; as under dry conditions, the extreme vapor pressure deficit (VPD) may trigger stomatal closure, leading 
to decreased nutrient acquisition mediated by mass flow (Abbate et al., 2004; Cramer et al., 2009).

Clearly, more research needs to be undertaken to better elucidate the role of microbes especially their diversity on food production and quality (Sinclair, 1992; St. Clair and Lynch, 2010). An integrated and holistic approach including controlled environment and field research is needed to investigate the long-term effects of manipulated soil biota communities on plant growth and mineral composition across soils with diverse abiotic characteristics. The emphasis should be to enhance our understanding of the plant-microbe interactions specific biota in their natural complex biotic and abiotic environment (Ladygina et al., 2010; Cortois and De Deyn, 2012).

In summary, agrobiodiversity (both plant and soil biota) is crucial to coping with climate change. The accumulated evidence reveals that there is a looming threat to biodiversity due to global warming as the organisms have to adapt to the changed environments to play their roles effectively in respective agroecosystems. The wild relatives among the plant species are at added risk due to genetic erosion and biodiversity loss. They need to be preserved in situ in protected areas to ensure evolution of new genetic variants, which may contribute to addressing new challenges to agricultural production and crop quality. The below ground biodiversity (soil biota) should also be explored, preserved (ex situ or in situ), and their role in coping with adverse effects of global warming on agriculture assessed, documented and knowledge disseminated. With the available modern instrumentation, it has become feasible to conduct accurate and cost-effective analysis of a large numbers of grain samples using high-throughput assays, allowing researchers to identify germplasm and breeding lines with desirable seed composition for subsequent use in crop breeding. These advances have led to the selection of various germplasm sources and new cultivars with desirable seed chemistry. However, very limited information is available globally on seed chemistry of germplasm collections. Hence, the need to screen core or mini core collections for seed chemistry to identify germplasm with improved seed composition for use in plant breeding programs. Soil fertility will be greatly impacted by both increase in atmospheric $\mathrm{CO}_{2}$ concentration and temperature. The effects of climate change via soil fertility and microbial diversity should be investigated on nutrient acquisition and utilization by crops, and their effects on food production and quality. The emphasis should be to enhance our understanding of plant-microbe interactions specifically of biota in their natural complex biotic and abiotic environments. 


\section{CLIMATE CHANGE ANALOG LOCATIONS REPRESENTING FUTURE CLIMATE}

Climates are changing rapidly elsewhere. This trend is likely to continue or even accelerate in the future, with likely serious, but variable consequences for agricultural productivity and quality around the world (IPCC, 2007; Challinor and Wheeler, 2008; Challinor et al., 2009; Boote et al., 2010; Moss et al., 2010; Palm et al., 2010; Reynolds, 2010). With climate change already posing a threat to food production around the globe, scientists are developing a form of virtual time travel that may offer farmers in various countries a glimpse of their future by the identification of regions and sites therein where the growing conditions at the present time match those that will exist in 2030 (Hallegatte et al., 2007; Kopf et al., 2008; Ramirez-Villegas et al., 2011). According to a recent report from the CGIAR Research Program on Climate Change, Agriculture and Food Security (CCAFS), the climate change analogs are today a view of tomorrow's agriculture, and will play a crucial role in adaptation research under climate change (RamirezVillegas et al., 2011).

The analogs approach in climate change research is a relatively novel way of supporting modeled recommendations with on-the-ground practical, empirical testing. The underlying principle is that the analogs tool connects sites with statistically similar or "analogous" climates across space in other geographic locations and/or time with historical or projected future climates. This approach helps to answer the key questions regarding climate change analogs: where sites can be found with similar climate at present or in the past or projected to be in the future analogous to the selected sites. In essence, a spatial analog is a location or site whose climate at present appears as a likely analog to the projected future climate of another site or location; and thus these two sites represent promising areas for conducting comparative research on adaptation plans in the face of climate change. On the other hand, temporal analogs are constructed using historical data that allow us to identify historical events that potentially can provide insights into the possible future consequences or fall-outs as a result of climate change. In addition, historical events provide an opportunity to learn as to how farmers adapted to climate shifts in the past. They equip farmers to deal with the changes that follow climate change at a site or in region. Although the current version of the analogs tool does not yet allow effective historical searches, the scientists involved in CCAFS are hopeful that this important 
function will be added in the future (Ramirez-Villegas et al., 2011). This tool is indeed an important development in facilitating adaptation through learning from analogous sites in both space and time.

The identification of analog sites coupled with relevant information collected from local field site studies or available databases can be employed and compared for developing further research studies including the analysis of the results obtained using simulating modeling (Hallegatte et al., 2007; Kopf et al., 2008) or for proposing high-potential adaptation pathways in a region (Ramirez-Villegas et al., 2011). Kopf et al. (2008) described a method to analyze the results of climate simulation models, which is an improvement on the method reported by Hallegatte et al. (2007). The proposed method is based on the concept of climate analogs, which in practical terms means finding a site (in this study a city) B whose present climate statistically corresponds to the simulated future climate of an evaluated site A.This exercise provides an intuitive visualization of the climate change effects on areas, by replacing the change of climate in time with a change of a site's location in space. Through the use of several models and scenarios, this methodology also helps to clarify the extent of the uncertainty in climate change prediction, and their impacts on targeted areas. The proposed method characterizes climate using three annual indicators-AI, heating degree days and cooling degree days-which are computed from monthly rainfall and temperature datasets. According to these authors, the methodology provides a better and strong basis for the visualization of climate change. Equally importantly, the proposed approach allows socioeconomic adaptation to different climates to enter the mental model for visualization (for details see Kopf et al., 2008).

By identifying and connecting analog sites in a network, research output can enable farmers to better envision as to how their site-specific agricultural future might look. Equally importantly, they can facilitate creating knowledge chain through which strategies or approaches and information regarding farming can be passed on or shared to facilitate more effective adaptation to climate change via interactions and learning. The analog tool also permits targeted field evaluation and testing of the climate resilience of crop production systems or specific technologies, and to identify effective technologies that can be implemented at analogous sites in the future. Furthermore, the analogous methodology allows the sharing and interpretation of historical data, and to learn lessons from case studies in an effort to building understanding of the best ways to improve climate resilience and enable adaptation to climatic changes (Ramirez-Villegas et al., 2011). Clearly, the use of climate analogs for locating future climates at the present time helps to 
ground models in field-based realities, significantly increasing our knowledge of adaptation capacity and identifying appropriate site-specific interventions. Although there is need for region-specific tweaks, validation processes, and to generate additional data for more robust results, the CCAFS analogs tools indeed offer important platform for future research and decision making relative to climate change adaptations (Ramirez-Villegas et al., 2011).

Haywood et al. (2011) discussed thoroughly the usefulness to benefit the society, of identifying a true prequaternary geological analog for future climate change. According to them, the identification of such sites provides a natural laboratory for evaluating numerical models of climate and the Earth system that are used to produce better predictions of future climate change. Indeed Earth history provides examples of how the planet responded to variations in atmospheric greenhouse gas concentrations in the longer term (several centuries). This methodology based on Earth history and using paleoclimate and environmental reconstruction and modeling are facilitating the assessment and calculation of the response of global temperatures to increasing carbon dioxide in multiple centuries' time span, also referred to as Earth system sensitivity, which derives climate change. Thus prequaternary geological analogs provide insight into future greenhouse warming and climate change with better precision (Crowley, 1990; Covey, 1995; Pagani et al., 2005; Haywood et al., 2011).

Despite the progress made in the use of climate analogs for adaptation to climate change, considerable uncertainty remains especially regarding projections of future climates and their impact on diverse farming systems at the local level. For example, the intrinsic adaptive and adaptation capacity of diverse rural communities is not taken into account in the global or regional-level models, which are used by policy makers for planning and implementation. Notwithstanding these limitations, the use of climate analogs for locating future climates at the present time can ground models in field-based realities. It is also important to note that the recently proposed CCAFS analogs tool offers an important platform for future research and decision making in the overall adaptation strategy to climate change (Ramirez-Villegas et al., 2011).

\section{PLANT PHENOMICS TO SCREEN TRAITS FOR ADAPTING TO STRESSES}

Precise phenotyping is the key to finding gene(s) and its allelic variants, analyze their expression, and thereafter for introducing these agronomically 
beneficial alleles into crop cultivars to enhance adaptation and meet new challenges to agricultural production. In the past, researchers have made significant progress toward developing conventional phenotypic screens for identifying novel sources of genetic variation, dissecting physiological and molecular basis of the response and adaptation to stresses, and finding alleles associated with beneficial traits. It is true that by using these phenotypic screens, crop genetic enhancers contributed substantially toward developing new cultivars in most of the agriculturally important crops. However, these screens involve large phenotyping costs, and are laborious, time-consuming, and are prone to errors due to variation in environmental conditions, with potential to severely limit the genetic gains.

Plant phenomics is the study of plant growth, performance and composition. It is a rapidly emerging area of research, offering a suit of new technologies to accelerate progress in understanding gene function and environmental responses, which will bridge the gap between genomics, plant function and agricultural traits, enabling breeders to develop improved germplasm or new cultivars with specific attributes (Furbank and Tester, 2011; Cabrera-Bosquet et al., 2012). High-throughput plant phenomics will also be beneficial to do research on crop water needs, fertilizer-induced deficiencies or other stresses including biotic and abiotic stresses, which will further help growers to take precautionary measures for intensifying sustainable crop production in commercial agriculture. Phenomics include both forward and reverse approaches, in which the former uses phenotyping tools to screen germplasm for valuable traits, while the latter dissects traits to discover their mechanistic understanding and allow exploitation of this mechanism to enhance the trait value into new germplasm. For this purpose various automatic high-throughput plant growth and phenotyping platforms have been established. They are known by the following names: PHENOSIS (Granier et al., 2006), PHENODYN (Sadok et al., 2007), GROWSCREEN (Walter et al., 2007), TraitMill ${ }^{\text {TM }}$ platform (Reuzeau et al., 2005), LemnaTec (http://www.lemnatec.com), and HTPheno (Hartmann et al., 2011), and are being progressively used in plants research and development, as detailed below.

\subsection{Root System Architecture}

Roots act as the interface between plants and soil. The root system architecture (RSA) refers to the temporal and spatial distribution and configuration of a root system in their natural undisturbed state within soil. RSA is a complex and difficult-to-quantify trait, and may differ between species. 
Knowledge of the root development and architecture therefore holds potential for manipulation of root traits to improve the productivity and sustainability of agricultural systems; and to better understand and manage natural ecosystems (De Smet et al., 2012). In the last 10 years, there has been considerable research done on understanding RSA in model plant Arabidopsis and a few agriculturally important crops such as common bean, maize, rice and wheat, which resulted in the development and application of a few phenotyping platforms and associated software for assessing RSA, including Shovelomics (Trachsel et al., 2010), RootChip (Grossmann et al., 2011), or nondestructive image-analysis toolbox for automatic phenotyping of RSA (Iyer-Pascuzzi et al., 2010), or X-ray computed tomography (CT) scanners for studying three-dimensional RSA (Fang et al., 2009; Clark et al., 2011; Flavel et al., 2012; Mooney et al., 2012).

Shovelomics is a high-throughput, low-cost, and easy to learn platform for phenotyping root architecture in maze in the field, which virtually scores 10 root architectural traits of the crown root of an adult maize plant in a few minutes. Trachsel et al. (2010) visually scored the number, angles and branching density of crown and base roots in three recombinant inbred lines (RILs) populations of maize, which provided reliable data as indicated by high correlations between measured and visually scored trait values for numbers $\left(r^{2}=0.46-0.97\right)$, angles $\left(r^{2}=0.66-0.76\right)$, and branching $\left(r^{2}=0.54-0.88\right)$ of base and crown roots. Further, using these visual scores, Trachsel et al. were able to discriminate between populations. For example, RILs derived from the cross between NY821 × H99 generally had the greatest number of roots, the highest branching density and the most shallow root angles, while inbred lines from the cross between $\mathrm{OH} 43 \times \mathrm{W} 64 \mathrm{a}$ generally had the steepest root angles. Moreover, the genotypes ranking remained the same across environments, emphasizing the suitability of the method to evaluate genotypes across environments. This platform should be directly applicable to other cereal crops such as sorghum or millet, while scoring system for nongraminaceous crops would have to account for a root architecture that is completely different than the one observed in maize. Thus, the visual evaluation of root architecture will be a valuable tool in tailoring crop root systems for specific environments (Trachsel et al., 2010).

Grossmann et al. (2011) reported a generic microfluidic chip platform, RootChip, which integrates live-cell imaging of growth and metabolism of Arabidopsis roots with rapid modulation of environmental conditions. It has separate chambers for individual regulation of the microenvironment of multiple roots from multiple seedlings in parallel, which can be modified for 
use with roots from other plant species, by adapting the chamber geometry to facilitate the systematic analysis of root growth and metabolism from multiple seedlings, paving the way for a large-scale phenotyping of root metabolism and signaling. This high-throughput root phenotyping assay provides a major advance for studying root biology with possibility of continuous imaging at the cellular and subcellular resolution for several days and under conditions that allow for root growth and root hair development. RootChip will greatly facilitate the ability to investigate nutrient uptake in different root zones, cell-type-dependent metabolite flux, and response of individual cells to different environmental stimuli.

The ability to nondestructively image and automatically phenotype complex root systems is fundamental to identifying genes underlying RSA. Iyer-Pascuzzi et al. (2010) reported a nondestructive image and analysis system for automated phenotyping and trait ranking of RSA in rice. Using 16 automatically acquired phenotypic traits for 2297 images from 118 individuals; Iyer-Pascuzzi et al. (2010) detected wide variation in phenotypes among genotypes and greater intergenotype than intragenotype variance of RSA, with option to integrate these data into computational pipeline that utilizes supervised learning methods to determine which traits best separate two genotypes. It also ranks the traits according to their contribution; and can assist to identifying candidate traits for automatic phenotyping of RSA in mapping populations. Further, Lobet et al. (2011) developed novel, semiautomated image-analysis software-SmartRoot - to facilitate the quantitative analysis of root growth and architecture of complex root systems, which combines a vectorial representation of root objects with a powerful tracing algorithm that accommodates a wide range of image source and quality. It also supports a sampling-based analysis of root system images. SmartRoot is an operating system-independent freeware based on Image and relies on cross-platform standards for communicating with dataanalysis software. Lobet et al. (2011) demonstrated the utility of this software to conduct time-lapse analysis of cluster root formation in lupin and maize root systems. Furthermore, Clark et al. (2011) developed a novel imaging and software platform, RootReader3D, for high-throughput phenotyping of three-dimensional root traits, and characterized 27 phenotypic root traits in rice. After validating these observations with two-dimensional measurements, they found them highly correlated. This highly flexible platform provides a capacity to measure root traits with a high degree of spatial and temporal resolution, which can facilitate novel investigations into the development of entire root systems or selected components of root systems, thus 
a powerful resource to explore the molecular and genetic determinants of RSA in crops.

X-ray CT, a nondestructive and noninvasive technique, offers new opportunity to monitor root growth and development in time and space in undisturbed environments (Mooney et al., 2012 and references therein). More recently, Flavel et al. (2012) reported high correlations between microCT observed roots and roots observed by standard methodology (soil core washing and WinRhizo analysis) in wheat. The potential drawbacks of this technology relate to the software to digitally segment roots from soil and air, which will improve significantly as automated segmentation algorithms are developed. A combination of rapid scans and automated segmentation will allow CT methodology to realize its potential as a high-throughput technique for the quantification of roots in soils.

\subsection{High-Throughput Imaging to Diagnose and Quantify Plant Response}

Phenomics has also advanced to develop other high-throughput phenomics technologies, a few of which include plant response to biotic and abiotic stresses (Matouš et al., 2006; Chaerle et al., 2007; Jones et al., 2009; Sirault et al., 2009; Berger et al., 2010; Munns et al., 2010; Furbank and Tester, 2011), dissecting dynamic changes in plant structure and functions (Jahnke et al., 2009), multisensor stress catalog (Chaerle et al., 2009), or module for handling large-scale phenotyping and genotyping data (Jung et al., 2011), which is beyond the scope of this chapter to provide procedural and technical details (Lenk et al., 2007). Nonetheless, we highlight their applications in agriculture including in plant breeding to developing cultivars with better adaptation to stress-prone environments.

Thermal and chlorophyll fluorescence imaging are powerful tools for the study of spatial and temporal heterogeneity of leaf transpiration and photosynthetic performance. Chlorophyll fluorescence imaging provides a powerful, noninvasive tool for investigating photosynthesis and its heterogeneity, and the variations in fluorescence transients could be used for early detection of biotic and abiotic stresses. Matouš et al. (2006) developed a new technique of combinatorial fluorescence imaging that significantly enhances our capacity to reveal dynamics of the plant-pathogen interaction. These images yield the highest contrast throughout the progression of infection in plants, which can be divided into segments that show tissue in different infection phases, as Matouš et al. demonstrated in case of Pseudomonas syringae infection in Arabidposis. The thermal and chlorophyll 
fluorescence imaging, when combined, provide specific signatures for diagnosis of distinct diseases and abiotic stresses, for example, rapid screening for stomatal responses can be achieved by thermal imaging, while, combined with fluorescence imaging to study photosynthesis, it can potentially be used to derive leaf WUE as a screening parameter (Chaerle et al., 2007). These images allow continuous automated monitoring of dynamic spatial variation. Moreover, such dual-imaging systems could be extended with complementary techniques such as hyperspectral and blue-green fluorescence imaging, which will increase the power of stress diagnosis and the potential for screening of stress-tolerant germplasm (Chaerle et al., 2007). Likewise, using hyperspectral and chlorophyll fluorescence imaging, Bauriegel et al. (2011) were able to detect wheat plots infected with head blight (F.culmorum). The disease severity was highly correlated with photosynthetic efficiency and above the infection limit of 5\% severity of disease, chlorophyll fluorescence imaging reliably detected infected ears.

Salinity is another major abiotic stress affecting crops production worldwide, which can be exacerbated by the changing climate. Sirault et al. (2009) developed a high-throughput automated image protocol that captures, identifies and analyzes thermal images acquired from a long-wave infrared (IR) camera to quantify the osmotic stress response of wheat and barley to salts. This technology in comparison to porometry (James et al., 2008) measures stomatal conductance through a precise, noninvasive and quick method, and needs only few seconds to acquire a thermograph comprising more than 3000 individual measurements for each object. It also takes into account spatial heterogeneity. IR thermal imaging could be therefore used to screen a large numbers of germplasm accessions, breeding lines and cultivars varying in the stomatal traits related to salt tolerance.

Drought is a complex stress that elicits a wide variety of plant responses. The nondestructive imaging techniques allow a temporal resolution and monitoring of the same plants throughout the experiment, which provides vital information on the physiological changes in response to drought over time to identify and characterize drought-response mechanisms into a series of component traits (Berger et al., 2010). A number of nondestructive imaging methods are now available to study physiological changes occurring in plants under drought, which include thermal IR imaging or IR thermography to assess transpiration rates (Trs), NIR to assess growth and water stress of plants, or fluorescence imaging to complement reflectance imaging. However, researchers should be aware of technological challenges that should be properly addressed to avoid erroneous results. It is thus clear that 
trait-dissection affected by high-throughput phenotyping could provide a significant new opportunity to enhancing our knowledge of plant response to drought, including elucidating the genetic basis of these responses and integrating such traits into appropriate combinations to improve crops performance under varying drought conditions (Berger et al., 2010; references therein; Furbank and Tester, 2011).

Agricultural production is limited by various abiotic and biotic stresses. Imaging technology has advanced to provide valuable information on plant stresses; and when supplemented by conventional video imagery (for study of growth), they provide an efficient early warning system to discriminate between different stressors to develop matrix that identifies specific signatures for multiple stress types, which allow agriculturists to reduce production losses in stress-prone environments (Chaerle et al., 2009).

Maize researchers took the lead to adapt some of the above highthroughput phenotyping tools to study variation in maize hybrids in response to drought under field conditions. Using high-throughput sensors, Winterhalter et al. (2011) demonstrated that canopy water mass (CWM, amount of water $\left.\mathrm{kg} \mathrm{m}^{-2}\right)$ correlates well $\left(r^{2}>0.70\right)$ with spectral indices and IR temperature with varying drought-stress levels, which enabled them to differentiate hybrids into three groups (above, below or average performer) under control and stressed environments. Their finding demonstrates that it is indeed possible to both detect CWM and discriminate between groups of maize hybrids using nondestructive high-throughput phenotyping, thus, potentially a useful technique for crop breeding. Resilience to soil water deficit and its genetics are a priority research area for maize breeding. More recently, Chapuis et al. (2012) tested and compared three methods of determining resilience to water deficit: the ability of hybrids to maintain leaf growth in a range of soil water potentials in a phenotyping platform, a direct estimator of resilience of seed number to water deficit in a network of field experiments, and classical methods involving adaptation to drought indices and variance analysis. They evaluated 19 maize hybrids by growing in 14 environments. Using the slope of regression line between drought index and seed number (which was taken as an estimate of the resilience to soil water deficit for each hybrid), Chapuis et al. (2012) detected twofold differences that correlated with resilience of leaf growth to soil water deficit in the phenotyping platform. Resilience estimated via genotype $\times$ watering treatment was nonsignificant due to large differences in drought indices between genotypes in a given watering treatment. This result led them to propose that the direct estimation of 
resilience to water deficit is feasible in the field with a minimum amount of environmental measurements.

O'Shaughnessy et al. (2011) used canopy temperature data to investigate whether an empirical crop water stress index (CWSIe) could be used to monitor spatial and temporal crop water stress in soybean and cotton. Using the benchmark relationships between CWSIe and leaf water potential $(\Psi \mathrm{L})$, they detected significant negative linear correlation between midday $\Psi \mathrm{L}$ measurements and CWSIe at well-established soil water differences. Average seasonal CWSIe values were inversely related to crop water use $\left(r^{2}\right.$ values $>0.89$ for soybean and 0.55 for cotton). O'Shaughnessy et al. (2011) also detected a significant inverse relationship between the CWSIe and soybean (2-year average $\left.r^{2}=0.85\right)$ and cotton $\left(r^{2}=0.78\right.$ in 1 year) yield, which enabled them to conclude that contour plots of CWSIe may be used as maps to indicate the spatial variability of within-field crop water stress; and thus may be useful for scheduling irrigation or identifying areas within a field where water stress may impact crop water use and yield.

\subsection{Sensor-Based Phenotyping Platform for Assessing Biomass}

High-throughput nondestructive biomass determination assay has a great potential to unravel the genetical, physiological and biochemical basis of plant growth. The traditional approach to phenotype biomass in the field is destructive, laborious, time consuming, and expensive. Tackenberg (2007) reported digital image analysis as nondestructive method to measure growth rate, fresh- and oven-dried biomass and its vertical distribution, and dry matter content, which is time saving and cost-effective. Recently, Montes et al. (2011) designed a high-throughput phenotyping platform that employs light curtains (LC) and spectral reflectance (SR) sensors mounted on a tractor to measure biomass in a field-grown experiment, spread over five environments, consisting of 20 maize hybrids. Using sensors (SR and $\mathrm{LC}+\mathrm{SR}$ ) and partial least square regression and support vector machine regression, Montes et al. (2011) found that biomass obtained by a combination of $\mathrm{LC}+\mathrm{SR}$ gave the lowest mean relative error of prediction Mean relative error (MRE) (0.11) and the highest $R_{v}^{2}$ (coefficient of determination of validation $=0.97)$, with high repeatability. Their research provided a proof-of-concept that this high-throughput, nondestructive phenotyping platform based on LC and SR sensors has a great potential for early biomass determination in field trials of maize and other space planted rowcrops. Furthermore, Golzarian et al. (2011) proposed a method for accurate 
estimation of plant shoot dry weight that employs information obtained from the images of plants and their age. It also provides an accurate and practical model for the estimation of shoot dry weight as a substitute for conventional destructive methods of biomass measurement in cereals.

Rascher et al. (2011) presented state-of-the-art phenotyping approaches to address aspects of resource use efficiency in plants, below ground roots, aboveground shoots and transport/allocation processes, using magnetic resonance imaging and automated fluorescence imaging (Pulse amplitude modulated (PAM) fluorometry) in combination with automated shape detection that allowed them doing high-throughput screening of photosynthetic traits. They are of the opinion that these phenotyping techniques together with mechanistic knowledge on the plant structure-function relationships will open new opportunities in whole-plant ecophysiology, which may assist in developing new germplasm with enhanced resources use efficiency.

\subsection{Developing Modules to Store, Retrieve, Add or Modify Large Datasets}

Technological development in high-throughput assays, mostly in genotyping but of late in phenotyping as well, allows researcher's phenotype and genotype large sets of individuals, which generate large datasets. Linking phenotypic variation and genotypic diversity is a major requirement for basic and applied genome-centric biological research. The mechanisms to be established should efficiently store, add, retrieve, or modify, query it, and analyze these datasets to derive meaningful interpretation between genotypic and phenotypic data. Currently, Chado, a generic, modular, community-based database is widely used in the biological community to store information associated with genome sequence data (http://gmod.org/ wiki/GMOD_Users). Its ontology-driven module allows researchers use the same schema in projects with widely different metadata, which can be modified or added, as new data types become available. Its modular design allows researchers select those modules needed to manage their datasets, and add to new modules as and when new dataset is generated. Chado is an open source, consists of 18 modules, and any user can contribute to schema (http://sourceforge.net/projects/gomd/), provided these contributions are consistent with the Chado generic design principle.

Recently, Jung et al. (2011) reported a new module, "ChadoNatural Diversity" module for storing large-scale phenotyping, genotyping and breeding data. This module strictly adheres to the Chado remit of being generic and ontology driven, and allows the storage of data from each 
experimental line that are scored for a large number of phenotypic traits, and genotyped with a set of genetic markers. It consists of tables for data storage from various experiments, and tracks the relationships of the experiments with data stored in other modules (http://gmod.org/wiki/Chado_ Natural_Diversity_Module). This module can store any type of experiment that either uses or generates specimens or stock organisms, which may be grouped or structured hierarchically, whereas any kind of biological entity can be stored as the observed unit, from a specimen to be used in genotyping or phenotyping experiments, to a group of species collected in the field that will undergo thorough laboratory analysis.

In spite of these recent developments, high-throughput phenomics research faces technological challenges to generate novel tools in computation and informatics. Its success will allow researchers to amass, access, integrate, organize, and manage phenotypic databases across species and conduct genome-wide analysis to associated phenotypic information for its applied value in agriculture (Lussier and Liu, 2007).

To sum up, it is clear that recent technological advances to developing high-throughput phenotyping platforms have taken a leap forward, and it is expected that more new innovations to refine these assays for multiple tasks, which in turn, will be useful in monitoring, diagnosing and taking corrective measures to plant growth and development to address yield-reducing constraints at the crop, community, regions or global scale. It is visualized that there will be increased interactions among the various stakeholders to use these resources, and tailor their germplasm with specific attributes to stress-prone environments.

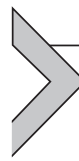

\section{PLANT TRAITS TO ACCELERATE ADAPTATION TO CLIMATE CHANGE}

\subsection{Genetic Enhancement for Adaptation to Abiotic Stress}

Plant breeders are often reluctant to use exotic germplasm including landraces, wild and weedy crop relatives, as these germplasm often show poor adaptation and low yield compared to modern cultivars. These germplasm may however possess desirable traits, e.g. host-plant resistance or adaptation to abiotic stresses. The use of exotic germplasm in breeding is a longdrawn process, often associated with undesirable traits; and shedding these negative linkage drags requires careful planning and execution of germplasm enhancement steps in such a way that the coadapted gene complexes, 
while introgressing new genes into improved genetic background, are not lost. Germplasm enhancement (also referred to as prebreeding by other authors) is a step before practical breeding for cultivar development. The products of germplasm enhancement are the intermediate (or semifinished) genetic materials that plant breeders can use to transfer desirable traits into an adapted genetic background. This process may take several years and following this plant breeders still have to develop commercial cultivars. Germplasm enhancement contributes to broadening the total genetic diversity in crops and provides specific traits to plant breeders. It is a vital link between conservation of plant genetic resources in gene bank collection and utilization of these resources in crops improvement.

CWR, which offer a rich source of genetic variation, are often grown in their natural habitats in conditions of climate and soil that may not be suitable for cultivation of modern crops. Evidence to-date suggests that CWR have provided many agronomically beneficial alleles which when brought into cultivated genetic background not only broaden the genetic base of cultigens and provided protection against biotic and abiotic stresses but also in some cases enhanced grain yield and quality attributes of crop cultigens. For example, Oryza officinalis, a source for early morning flowering trait, has been used to change the time of flowering of cultivated rice (Oryza sativa), which can mitigate yield loss due to global warming by escaping high-temperature stress at anthesis during the day time (Ishimaru et al., 2010), Moreover, CWR contribute to hostplant resistance to many pests and pathogens in both cereals and legumes (Dwivedi et al., 2005, 2008 and references therein). Furthermore, hybrid rice in China became reality when male sterility was discovered in a wild-rice O. sativa f. spontanea (Yuan, 1993). Similarly, nuclear genes from Cajanus acutifolius interact with pigeonpea (Cajanus cajan) to produce cytoplasmic nuclear male sterility system in pigeonpea (Dwivedi et al., 2008 and references therein). Likewise, wild species contribute yield and quality enhancing alleles in rice and tomato (Dwivedi et al., 2008 and references therein).

Hexaploid bread wheat resynthesis can be done by crossing tetraploid durum cultivars with diploid wild relatives (including nonprogenitor wheat species), enabling a whole new gene pool to be introduced that did not come from the original wild crosses from which the first ancient wheat ensued. These resynthesized wheats provided rich source of diversity for adaptation to stresses to the wheat cultigen pool (Dwivedi et al., 2008 and references therein). 
Simpson et al. (1993) were probably the first to produce fertile amphiploid, peanut (TxAG-6) by colchicine treatment of the sterile triploid obtained from crossing the AA-genome donor hybrid (Arachis cardenasii $\times$ A. diogoi) with the BB-genome species, Arachis batizocoi. It is thus a useful resource for introducing genetic variability into the peanut cultigens. Using this approach, two peanut cultivars Coan and NemaTAM carrying genes for root-knot nematode (Meloidogyne arenaria) resistance from A. cardenasii have been released for cultivation in the USA (Simpson and Starr, 2001; Simpson et al., 2003a). Likewise, researchers in Brazil and India have produced a range of new amphidiploids, that when doubled the chromosomes, produced fertile synthetics (Mallikarjuna et al., 2011; Fonceka et al., 2012). They are expected to release new source of variation for use in genetic improvement of peanut. For example, Fonceka et al. (2012) detected many wild alleles that contributed positive variation to several traits (pod and seed size, pod maturity and biomass production) involved in peanut productivity and adaptation. Likewise, breeding lines with higher 100-seed weight (up to $95 \mathrm{~g}$ ) compared to $32 \mathrm{~g}$ of cultivated parent TMV 2, and with 23-68\% more pod yields than TMV $2\left(3343 \mathrm{~kg} \mathrm{ha}^{-1}\right)$ were selected in a segregating population involving TMV 2 and TxAG-6 (H. D. Upadhyaya, ICRISAT, unpub). TxAG-6 yielded $2-5$ g plant $^{-1}$ with very low 100 -seed weight $(\sim 10 \mathrm{~g})$. These results demonstrated that the novel alleles of CWR, which were considered to be lost in evolution to cultivated types, could be used to enhance important agronomic traits in cultivar (Upadhyaya, 2008).

Developing exotic genetic libraries, also known as introgression lines, consisting of marker-defined genomic regions taken from wild species and introgressed onto the background of elite crop cultivars is another approach suggested to enhance trait variability for use in crop improvement (Eshed and Zamir, 1994). Such an approach has been widely employed in tomato genetics and breeding for transferring desirable traits in cultivated tomato. For example, pyramided tomato introgressed lines containing three independent yield-promoting genomic regions produced more than 50\% greater yields compared to controls under both wet and dry field conditions (Gur and Zamir, 2004). Likewise, introgressed progenies containing yieldenhancing alleles from wild rice produced higher grain yield in rice (Xiao et al., 1996).

Clearly, CWR are excellent source of genetic variation for agronomically beneficial alleles that when brought together into cultivated genetic backgrounds have the potential to enhance trait value(s). However, useful traits from interspecific crosses will often carry negative linkage drag, thereby 
making the use of CWR a long-term process in breeding programs because of the lengthy process in which relevant traits are gradually transferred to an elite background. A long-term consortium-based approach involving conservationists, geneticists, plant breeders, and molecular biologists may be able to harness the wealth of CWR to address agriculturally important problems, more so in this time of climate change.

\subsection{Integrating Trait Diversity to Develop Climate-Proof Nutritious Crops}

\subsubsection{Drought Adaptation in Cereals}

Breeding for adaptation to drought-prone environments is extremely challenging due to the complexities associated with various stress-adaptive mechanisms, uncertainties in onset of stress, and large genotype $\times$ environment interaction. A detailed review of progress toward understanding the mechanisms of adaptation to drought, the component traits associated with it, their genetic control, and the genomic regions quantitative trait locus (QTL) associated with such adaptation in barley, maize, pearl millet, rice, sorghum and wheat has been made elsewhere (Dwivedi et al., 2010). Here, only briefly report on current progress to further enhance our understanding of physiological and molecular basis of adaptation to drought and how this knowledge is being employed to develop crops targeting droughtprone areas using conventional and applied genomic tools.

\subsubsection{Rice}

Bernier et al. (2007) detected a major QTL, qtl12.1 on chromosome 12 in Vandana/Way Rarem mapping population, which contributed 51\% phenotypic variance for grain yield under drought over two dry seasons. The drought-susceptible parent, Way Rarem, contributed alleles that confer advantage to improved yield under drought-stressed conditions. Subsequent work on large-scale evaluation of a subset of 100 lines, 50 homozygous for the positive (Way Rarem-derived allele) allele and 50 for the negative (Vandanaderived allele) allele, across locations in the eastern India and the Philippines for 2 years, revealed that relative effect of the QTL on grain yield increased with increasing intensity of drought stress, from having no effect under wellwatered conditions to having an additive effect of more than $40 \%$ of the trial mean under severe stressed conditions (Bernier et al., 2009).

Guan et al. (2010) characterized a set of 48 rice-pyramiding lines (PLs), obtained through QTL pyramiding ( $\mathrm{Li}$ and $\mathrm{Xu}, 2007$ ), and their recurrent parent, IR64, under drought-stress and nonstress conditions. In comparison 
to IR64, all PLs had significantly improved adaptation to drought at reproductive stage, while 36 PLs also had significantly improved adaptation to drought at vegetative stage. Seventeen PLs had higher yield than IR64 under drought stress, while the remaining 31 PLs yielded at par with IR64 under irrigated conditions. The characterization of these PLs further revealed that dehydration avoidance, efficient partitioning high harvest index (HI) and drought escape (early flowering) together contributed to their improved adaptation to drought. These results indicate that the selection for yield together with some secondary traits under appropriate type(s) of stress and nonstress conditions similar to the target environments are critically important for improving adaptation to drought without any yield penalty in rice. Adaptation to drought has also been found related to deep root growth and water uptake ability in both upland and lowland rice agroecologies (Gowda et al., 2011). More recently, Gowda et al. (2012) evaluated a set of 20 diverse rice genotypes, identified as Oryza single-nucleotide polymorphism (SNP) panel, for root water uptake ability as a candidate trait for response to drought in greenhouse lysimeter experiments. They detected large genotypic differences in water uptake and plant growth in response to drought. The total water uptake and water uptake rates correlate with relative root length density, especially at depth below $30 \mathrm{~cm}$, which reveals that response to drought by deep root growth, rather than a conservative soil water pattern, is important for lowland rice, with aus rice genotypes showing greatest values for water uptake and root growth. It will be interesting to investigate possible association between root traits and grain yield under drought stress.

Rice cultivars such as Sahbhagi Dhan, Sahod Ulan 1, and Tarharra 1, developed through conventional breeding and selections, have been recently released because of their enhanced adaptation to drought-prone environments of India, the Philippines and Nepal, respectively. They are being disseminated to farmers in drought-prone areas in these countries. These cultivars have shown yield advantage of $\sim 1 \mathrm{t} \mathrm{ha}^{-1}$ under stress (Mackill et al., 2012). Recent study on the new rice for Africa (NERICA) rice cultivars, developed by crossing Asian rice (O. sativa L.) and African rice (Oryza glaberrima Steud.) and released for cultivations in sub-Saharan Africa, show that these NERICA rices do not consistently provide the expected combination of superior yield potential with strong weed-suppressive ability and overall adaptation to low soil fertility conditions usually found in the target regions. The yielding ability of these upland NERICA cultivars tested to date is similar to one of their O. sativa parents, WAB56-104, whereas they have inferior ability to suppress weeds to their Oryza glabarata parent; and they 
also have lower adaptability to low soil fertility than aerobic rice and aus cultivars, thus a clear need to further improve the NERICAs (Saito et al., 2012 and references therein). Saito et al. (2012) also identified some aerobic rice and aus cultivars with high-yielding ability, strong weed competitiveness, and superior adaptation to low soil fertility, and thus would seem ideal resource for the development of upland interspecific cultivars adapted to sub-Saharan African conditions.

Grain yield under drought is a complex trait, and grain yield heritability in drought-prone environments is relatively low compared to irrigated control (Venuprasad et al., 2007). Gouda et al. (2012) evaluated O. sativa IR 58025B $\times$ Oryza meridionalis $\mathrm{BC}_{2} \mathrm{~F}_{3}$ derivatives under water stress to estimate heritability for grain yield and identify physiomorphological traits associated with enhanced adaptation to drought. The broad-sense heritability for grain yield under water stress was 33\% compared to 59\% under irrigated control. From this evaluation, they identified 20 high-yielding lines that together with 40 randomly selected lines they further evaluated under control (irrigated) and stress conditions to estimate selection response. In addition, they also evaluated 10 lines (five each from high- and low-yielding groups) for root morphological traits. This study revealed that high-yielding lines had recorded maximum root length and root volume compared with checks and low-yielding lines, indicating positive influence of drought avoidance root traits contributing to stress tolerance, and selection for grain yield under stress is an effective strategy in deriving rice lines with enhanced adaptation to drought.

\subsubsection{Wheat}

A technique based on spectral reflectance indices (SRI) has been proposed as a potential methodology to assess canopy biomass, leaf area index, light absorption, photosynthetic activity, and for predicting yield by periodic measurements of reflectance during the plant development in crops (Rudorff and Batista, 1990; Wiegand et al., 1991; Peñuelas, 1998; Reynolds et al., 1999). The most commonly known index for analyzing vegetation is the NDVI (Araus et al., 2001; Osborne et al., 2002; Royo et al., 2003; GutierrezRodriguez et al., 2004; Prasad et al., 2007a,b). Gutierrez-Rodriguez et al. (2010) determined the relationship between the SRI and yield of the spring wheat in irrigated, water-stressed, and high-temperature environments using three and five SRI, respectively; and for this purpose data on vegetative and water indices were collected at booting, heading, and grainfilling stages of the crop in advanced lines in three trials. This study revealed 
that two water indices (NWI-1 and NWI-3) consistently provided high correlations with grain yield when the data on the indices at heading and grain-filling stages were combined in all the three trials. Vegetative indices showed inconsistency in their relationship with grain yield. The water indices gave higher genetic correlations and correlated response with grain yield than the vegetative indices. Likewise, there was a strong relationship between grain yield and canopy temperature determined at grain filling. The high-temperature environment showed the strongest association of the SRI with grain yield. It thus is clear that water indices can be used for plant breeding purposes for selecting high-yielding lines in well-irrigated, water-stressed and hot environments; and the canopy temperature could complement this selection.

\subsubsection{Maize}

Farmers' fields in southern Africa are rarely characterized by only one abiotic stress. An open pollinated or hybrid cultivar must combine adaptation to drought and low-N stress, with high yield potential under optimal conditions to become popular among farmers (Bänziger et al., 1999; Kumar et al., 2008). Selection under abiotic stress, optimal, and/or low-N conditions allows identification of broadly adapted genotypes. CGIAR Consortium researchers and partners evaluated large number of elite hybrids under optimal, low-N, and manage drought conditions during the 2001 and 2009 crop seasons to predict the performance under abiotic stress and low-N fertility usually faced by African farmers. This study reveals high genetic correlations between abiotic stress and low- $\mathrm{N}$ or optimal conditions. The indirect selection under low- $\mathrm{N}$ and optimal conditions is therefore more efficient than direct selection under abiotic stress or indirect selection under managed drought, especially for early maturing genotypes. Direct selection is however most efficient for predicting performance under low $\mathrm{N}$. Elite hybrids tolerant to abiotic stress can be most efficiently selected under optimal or low-N conditions while low-N-tolerant genotypes should be selected directly under low N (Weber et al., 2012).

Conservative water use early in the growing season particularly under high atmospheric VPD is an important adaptive strategy to save water for the later growing season to support grain development. The partial closure of stomata under high VPD decreases the effective average daily VPD for transpiration allowing more efficient use of available water in the later growing season (Sinclair et al., 2005).Yang et al. (2012) studied the response of $\mathrm{Tr}$ to changes in atmosphericVPD at two different temperatures in maize 
hybrids in a growth chamber. Some hybrids in this study showed limitation of increase in Tr at high VPD above a break point (BP), which could operate through anatomical or physiological features; thus seems an important adaptive strategy to improve adaptation to drought in maize. Further research to better understand the mechanism of transpiration regulation that will assist maize breeding to improve adaptation to drought by incorporating the transcription restriction trait is suggested.

\subsubsection{Sorghum}

The stay-green trait (i.e. reduced leaf senescence) improves tolerance to postflowering moisture stress in sorghum.Various QTL associated with staygreen have been reported, but only a few have a major effect on trait expression in sorghum. The QTL-introgressed lines produced significantly higher grain yield under moderate water stress than irrigated control (Kassahun et al., 2010; Dwivedi et al., 2010 and references therein), with majority of the introgression lines having higher leaf chlorophyll levels at flowering and a greater percentage green-leaf area during the latter part of grain filling (Kassahun et al., 2010). Recently, Jordan et al. (2012) investigated the relationship of the stay-green with grain yield using data from sorghum breeding trials that sampled 1668 unique hybrids and 23 environments in which the mean grain yield varied from 2.3 to $10.5 \mathrm{t} \mathrm{ha}^{-1}$ in Australia. The strength and direction of the associations between stay-green and grain yield varied with both environment and genetic background (male tester). The majority of the associations were positive, particularly in environments with yields below $6 \mathrm{t} \mathrm{ha}^{-1}$. As the trial mean increased above $6 \mathrm{t} \mathrm{ha}^{-1}$ there was a trend toward an increased number of negative associations. However, the number and magnitude of the positive associations were larger. These results indicate that selection for stay-green in elite sorghum hybrids may be broadly beneficial for increasing yield in a wide range of environments.

\subsubsection{Pearl Millet}

The research to-date suggests that genotypes that flower early possess fewer but effective basal tillers, are low in biomass, and have high HI perform better under terminal drought stress. A major QTL on linkage group 2 is associated with postflowering adaptation to drought in pearl millet (Dwivedi et al., 2010 and references therein). The physiological basis of this QTL for adaptation to drought reveals that water conserving mechanisms, mostly expressed under nonstressed conditions allow water saving in the soil profile during the early crop stage, which is available to the plant during 
postanthesis period (Kholová et al., 2010a,b). Recently, Kholová et al. (2012) evaluated RILs involving H77/833-1 (sensitive to terminal drought) and PRLT2/89-33 (tolerant to terminal drought) to map Tr and associated physiological traits. Two alleles each from H77/833-1 and PRLT2/89-33 on linkage group 2 increased Tr, whose importance depended on the VPD. More importantly, the two H77/833-1 and one PRLT2/89-33 alleles in this study comapped to a previously identified major terminal droughttolerant QTL, while the other Tr allele from H77/833-1 enhances biomass dry weight and colocated with a previously identified stover and tillering QTL (Yadav et al., 2002, 2004). Leaf traits were linked to two loci on linkage group 7. Kholová et al. (2012) also detected variable plant water use depending on allelic combinations for Tr, tillering and leaf characteristics, whose importance depended on the environmental conditions. These authors therefore concluded that different alleles influence plant water use, and have close interactions with one another and with the environment, which may be used to design plant ideotypes from specific allele combinations, conferring particular physiological traits for specific adaptation to a range of terminal drought conditions.

\subsubsection{Submergence and Phosphorus Deficiency Tolerance in Rice}

A large part of the rainfed lowland rice acreage in South and Southeast Asia is affected by submergence of varying degree and duration, resulting into substantial loss to rice production in these regions. FR13A and a few other landraces from India and Sri Lanka have been identified as sources of complete submergence, with FR13A an extremely submergence tolerant. A major QTL on chromosome 9 in FR13A, Sub1, accounting for $69 \%$ of variation, provides tolerance to complete submergence for up to 2 weeks (Xu and Mackill, 1996). Subsequent work on fine mapping of this QTL delineated Sub1 to a genomic region of approx. $0.06 \mathrm{cM}$ (Xu et al., 2006). The sequencing of Sub1 region in an FR13A-derived line revealed the presence of three genes encoding putative ethylene responsive factors, Sub1A, Sub1B and Sub1C, with Sub1A identified as the major determinant of submergence tolerance in rice (Xu et al., 2006). Using flanking markers and marker-assisted breeding, researchers have introgressed Sub1A into popular high-yielding cultivars from Bangladesh, India, Laos and the Philippines. To-date, a number of submergence-tolerant mega cultivars such as Swarna-Sub1, SambaMahsuri-Sub1, Thadokkam1-Sub1, BR11-Sub1 and IR64-Sub1 have been released in Bangladesh, India, Indonesia, the Philippines and Sri Lanka (Neeraja et al., 2007; Sarkar et al., 2009; 
Singh et al., 2009; Septiningsih et al., 2009; Bailey-Serres et al., 2010; Iftekharuddaula et al., 2011). These cultivars with Sub1 tolerate complete submergence for 2 weeks (Das et al., 2009), whereas a few other germplasm could withstand 3 weeks of complete submergence with greater variation in plant height and elongation ability under submergence (Sarkar and Bhattacharjee, 2012). Encouraged with these successes, rice breeders are now using Sub1 locus to develop submergence-tolerant cultivars for submergence-prone areas in Africa and Asia.

Phosphorus is the second most important plant nutrient only after nitrogen; and phosphorus availability is limited because applied soluble phosphorus is converted into insoluble form due to phosphate sorption by soil minerals and or due to reactions to form iron- and aluminum-phosphorus compounds in acid soils, and calcium- and magnesium-phosphorus compounds in alkaline soils. The availability of phosphorus is optimum around a neutral pH in soils (Sahrawat et al., 2001; Sparks, 2003). Phosphorus fertilizers are manufactured from phosphate rock, a nonrenewable resource, and thus likely to deplete in 50-100 years. The quality of phosphate rock is declining and production cost is increasing. About $\sim 60 \%$ of rainfed rice in Asia is grown on soils that are affected by multiple stresses, including P deficiency (Haefele and Hijmans, 2007; Sahrawat, 2009). Genotypic differences in rice have been reported for tolerance to phosphorus deficiency (Wissuwa and Ae, 2001a). A major QTL, Phosphate uptake 1 (Pup1) on chromosome 12, confers tolerance to phosphorus (P) deficiency in soil (Ni et al., 1998; Wissuwa et al., 1998, 2002). The evaluation of near isogenic lines (NILs) with and without the Pup1 QTL showed that Pup1 increases P uptake (Wissuwa and Ae, 2001b; Wissuwa et al., 2002), confers a significant yield advantage in P-deficient soils and mostly conserved in germplasm or cultivars adapted to drought-prone environments (Chin et al., 2010). Recently, Pup 1 has been the target for development of tolerant rice cultivars. Chin et al. (2011) introgressed Pup1 into five cultivars, adapted in irrigated or rainfed agroecology. The phenotypic evaluation of the introgression lines suggest that Pup1 is effective in different genetic backgrounds and environments and that it has the potential to significantly enhance grain yield under phosphorus deficient soils. Ismail et al. (2007) indicated that ongoing rice genetic resources and breeding research aims identifying germplasm tolerant to problem soils, dissect the genetics of component traits conferring advantage of tolerant germplasm on these soils, fine map the genomic region(s) using gene-based markers, and transfer these beneficial alleles conferring tolerance to nutrient deficiency into improved genetic backgrounds. 


\subsubsection{Adaptation to Drought in Legumes}

\subsubsection{Soybean}

Studies in cereals have clearly demonstrated that a limitation on $\mathrm{Tr}$ at high VPD allow soil water conservation during vegetative growth stage that the plants uses during the postanthesis period to maximize yield under moisture stress conditions (see Section 8.2.1). Using a simple experimental system in which whole plant Tr could be measured while subjecting plants to a wide range of VPD conditions, Fletcher et al. (2007) identified a slowwilting soybean germplasm PI 416937 with a two-segment Tr response to VPD; i.e. above a BP of $\sim 2 \mathrm{kPa}$, Tr remains constant. Further study on this unique response of PI 416937 to increasing VPD revealed that there is a $\mathrm{BP}$ in this genotype at about $2 \mathrm{kPa}$ and that the limitation on $\mathrm{Tr}$ at higher VPD is linked to low hydraulic conductance at the leaflevel, which is absent in genotypes that had no BP in Tr in response to VPD (Sinclair et al., 2008). Sadok and Sinclair (2009) assessed Tr response in soybean genotypes having wide genetic base and identified additional two genotypes (N73-1102 and NTCPR 94-5157) as expressing a BP in Tr response to VPD. More importantly, these two genotypes displaying the two-segment $\operatorname{Tr}$ response to VPD are not derived from PI 416937; however, these showed a similar BP value (approximately $2 \mathrm{kPa}$ ) as of PI 416937. Recently, Sinclair et al. (2010) used a relatively simple, mechanistic soybean growth and yield model (Sinclair, 1986) and 50 years of weather data for 2655 US grid locations of $30 \mathrm{~km}$ by $30 \mathrm{~km}$ size to assess the yield response to modification of root depth, rate of leaf area development, decreased stomata conductance at high soil water content, reduced maximum Tr, and drought-tolerant nitrogen fixation. The simulation results reveal that both water conservation during early growth decreases stomata conductance with soil drying and by reducing the maximum Tr: i.e. slow-wilting phenotype, resulted in increased yield, with high probability of yield gains for much of the soybean production areas in $70 \%$ or more of the years. The genetic material carrying slow-wilting trait and early stomata closure thus have the potential to improve germplasm for yield beyond the current levels observed in breeding programs for much of the soybean production. Further, the drought-tolerant nitrogen fixation had the greatest benefit of all traits with yield gain in more than $85 \%$ of the years at almost all locations in soybean, which should also be incorporated along with slow wilting and early stomata closure into soybean improvement programs. Clearly, identification of these germplasm with water saving adaptive trait is an ideal genetic resource for enhancing adaptation to drought in soybean. 


\subsubsection{Peanut}

Several sources of adaptation to mid- and/or end-of-season terminal drought, both in subspecies hypogaea and fastigiata, have been reported in peanut, which showed variation for physiological traits such as specific leaf area, chlorophyll content, amount of water transpired (T), transpiration efficiency (TE), WUE, and HI under drought-stress conditions (Upadhyaya, 2005; Dwivedi et al., 2007b; references therein; Jyostna Devi et al., 2009a). Most of the peanut breeding programs follow an empirical approach, which was largely based on pod yield as selection criterion for adaptation to drought, resulting thereby in slow progress. Nigam et al. (2005) were the first to evaluate the relative efficiency of physiological trait-based selection vis-à-vis empirical selection for adaptation to drought in peanut. The physiological trait-based method did not show a consistent superiority over the empirical method of breeding for drought adaptation for producing high kernel yield in peanut. Nonetheless, the integration of physiological traits (or their surrogates) in the selection scheme would be advantageous in selecting genotypes that utilize water more efficiently and partition the photosynthates more effectively into economic yield. When evaluated for variation in TE under progressive soil drying conditions, Jyostna Devi et al. (2009a) detected no differences in TE among 17 genotypes under well-watered conditions. However, under soil drying conditions there were substantial differences among genotypes. This indicates that TE with drying soil might interact with traits associated with water loss on drying soils. The genotypes showed large variability for fraction transpirable soil water (FTSW) content (0.22-0.71), and a decline in transpiration with soil drying. This difference in FTSW in peanut is much greater than those reported for other plant species (Jyostna Devi et al., 2009a and the references cited therein). The existence of a break point in the $\mathrm{Tr}$ at the elevated VPD offers the opportunity to directly influence crop transpiration use efficiency under field conditions. Jyostna Devi et al. (2010) evaluated 17 tolerant germplasm with large variation in TE and detected genotypic variation in $\mathrm{Tr}$ in response to high VPD. Nine of these genotypes showed a BP of about $2.2 \mathrm{kPa}$, above which there was little or no further BP increase in $\mathrm{Tr}$, which reveals that these genotypes with a $\mathrm{BP}$ have the potential to soil water conservation when VPD exceeded $2.2 \mathrm{kPa}$. This trait will be useful in terminal drought conditions as genotypes with a BP will be able to make use of conserved water to generate a greater yield than genotypes without the BP. It should be noted that decrease in stomata closure associated with decreased $\operatorname{Tr}$ would adversely affect photosynthesis. However, this loss may be offset by water savings in the soil due to risingVPD for use later 
in the season, resulting greater yield. Furthermore, the identification of few major, many minor and epistatic QTL reveals that the adaptation to drought is complex and multigenic in peanut (Ravi et al., 2011).

\subsubsection{Chickpea}

Chickpea is among the most studied legume crop for response to drought. Adaptation to drought has been found associated with variation in drought avoidance root traits (root length density, root to total dry plant weight ratio, root depth, and root to shoot length density), TE, carbon isotope discrimination $\left(\delta^{13} \mathrm{C}\right)$, Soil plant analysis development (SPAD) chlorophyll meter reading (SCMR), and canopy temperature (Kashiwagi et al., 2005, 2006, 2008 , 2010). Drought escape as a result of early maturity is another important adaptive mechanism, which has opened up new possibilities for growing chickpea in semiarid and arid regions globally (Kumar and Abbo, 2001). Conservative use of water at vegetative stage saves water for use by the crop during the reproductive stage. Zaman-Allah et al. (2011a) evaluated 20 chickpea genotypes with similar phenology but differing in response to terminal drought stress. The pattern of water extraction in their study clearly discriminated tolerant and sensitive genotypes. Tolerant genotypes had a lower water uptake and a lower index of stomatal conductance at vegetative stage than the sensitive ones, while genotypes with enhanced adaptation to drought extracted more water than sensitive genotypes after flowering. Further, the magnitude of the variation in root traits did not distinguish the tolerant from the sensitive genotypes. The seed yield was not significantly correlated with root traits, whereas seed yield was negatively related to water uptake between 23 and 38 days after sowing (DAS), and positively related to water uptake between 48 and 61 DAS. Zaman-Allah et al. (2011a) therefore concluded that under terminal drought conditions, conservative use of water early in the cropping cycle is the most critical component of adaptation in chickpea, partly as a result of a lower canopy conductance, which resulted in more water available in the soil profile during reproduction leading to higher reproductive success. Zaman-Allah et al. (2011b) further tested whether plant water use at the vegetative stage and under no-limiting water conditions could relate to the degree of sensitivity of chickpea to terminal drought. They found that the genotypes with enhanced adaptation to drought had a lower canopy conductance under fully irrigated conditions at vegetative stage, while the trend reversed at the early pod-filling stage. The sensitive genotypes had high early growth vigor and leaf development, while the genotypes adapted to drought had 
lower growth under progressive soil drying. The genotypes with enhanced adaptation to drought also exhibited decreased transpiration in wetter soil compared to sensitive genotypes. These results suggest that some traits contribute to water saving when water does not limit plant growth and development in chickpea with enhanced adaptation to drought. It is encouraging to know that chickpea breeders are now using these traits to enhance performance in drought-prone environments. Of recent, a large number of chickpea germplasm with enhanced adaptation to terminal drought and heat stress have been reported in literature (Kashiwagi et al., 2005, 2006, 2008, 2010; Krishnamurthy et al., 2010; Upadhyaya et al., 2011d; Zaman-Allah et al., 2011a,b). Chickpea genomics research has advanced considerably with the availability of large number of microsatellites and SNP (Varshney et al., 2007, 2009a; Nayak et al., 2010), fairly covered genetic maps (Millan et al., 2010; Nayak et al., 2010), and a large number of Expressed sequence tag (EST) resources involved in responses to drought and salinity (Varsheny et al., 2009b). Molecular biologists are working with crop physiologists and plant breeders to identify genomic regions associated with enhanced adaptation to both drought and salinity. Their research success will speed up chickpea breeding for stressful environments.

\subsubsection{Cowpea}

The high productivity of some of the cowpea landraces such as DWDCC 015, DWDCC 006 and DWDCC 001 reflects the ability of these landraces to tolerate and respond to a wide range of temperature- and moisturestress conditions (Hegde and Mishra, 2009). These and other germplasm with enhanced adaptation to drought and heat produce more pods and seeds plant ${ }^{-1}$, grain yield plant ${ }^{-1}$ and show high HI (Ehlers and Hall, 1998; Hall, 1992, 2004; Hegde and Mishra, 2009). Breeding efforts have been successful toward developing several cultivars that have been released globally (Cisse et al., 1995, 1998; Ehlers et al., 2000; Ismail et al., 2000; Elawad and Hall, 2002; Hall et al., 2003; Hall, 2004). More recently, genotypic differences in water-saving traits: i.e. lower $\operatorname{Tr}$ under no stress and restricted Tr under high VPD offer opportunities to enhance drought adaptation in cowpea (Belko et al., 2012). Delayed leaf senescence in IT93K-503-1 and IT98K-499-39 is associated with adaptation to drought (Muchero et al., 2008). Muchero et al. (2009) evaluated RIL populations, derived from IT93K503-1 (showing enhanced adaptation to drought) and CB 46 (susceptible), in the greenhouse and fields. They detected 10 QTL associated with seedling drought-induced senescence, mapped on linkage groups 
$1,2,3,5,6,7,9,10$, and with each contributing between $5 \%$ and $12 \%$ to the phenotypic variance. The stability of QTL expression across time and environment in their study further suggests that the genomic regions harboring the identified QTL carry genes that are of major importance in determining cowpea response to drought. The drought-induced senescence QTL (except Dro-8) was independent from eight QTL for maturity on linkage groups 7 and 8 . This finding suggests that it should be possible to pyramid early maturity with delayed drought-induced senescence to manage both early and late-season drought in cowpea. Interestingly, several RILs in this study expressed combined traits of early maturity with delayed droughtinduced senescence.

\subsubsection{Common Bean}

Common bean has two major gene pools-Andean and Mesoamericaneach with three races: Chile, Nueva Grenada and Peru in the former and Durango, Jalisco and Mesoamerica in the latter (Beebe et al., 2000). Sources and plant traits associated with enhanced adaptation to drought in common bean have been identified in both the Mesoamerican and Andean gene pools (Acosta-Gallegos and Kohashi-Sibata, 1989; Acosta-Gallegos and Adams, 1991; Acosta-Gallegos and White, 1995; Singh, 1995; Ramirez-Vallejo and Kelly, 1998; Terán and Singh, 2002; Muñoz-Perea et al., 2006). Traits that confer enhanced adaptation to drought in common bean include a deep rooting system with appropriate architecture that increases extraction of soil moisture from a greater soil depth; maximization of WUE for photosynthesis, growth and development; greater photosynthates transport to seed under stress through efficient remobilization; early maturity; and recovery from drought. Several germplasm and breeding lines possessing a combination of these traits have been either identified from gene pools or developed by crossing and selection in many bean breeding programs around the world (Frahm et al., 2004; Micklas et al., 2004; references therein; Beebe et al., 2008; Acosta-Diaz et al., 2009; Asfaw et al., 2012a). As is the case with peanut (Nigam et al., 2005), seed yield (empirical selection) shows greater genotype $\times$ environment interaction than functional traits such as the transpiration, WUE, and HI. However, the negative correlation between these traits may limit genetic progress using trait-based selection (Dowkiw et al., 2000). Some recently bred lines from the CGIAR Consortium outyielded the commercial checks by $15-25 \%$ under favorable environments or up to $36 \%$ under drought stress. Some of these lines also produced more grains in phosphorus-limited environments, which indicate 
the potential to combining traits enhancing adaptation to drought and tolerance to phosphorus deficiency in common bean (Beebe et al., 2008). Enhanced adaptation to drought is multigenic in common bean and adoption of recurrent selection is an effective breeding strategy (Beebe et al., 2008). Few QTL associated with enhanced adaptation to drought has been identified (Schneider et al., 1997; Blair et al., 2012). Improved photosynthate acquisition, accumulation, and remobilization have been observed as important mechanisms for adaptation to drought stress. Asfaw et al. (2012b) evaluated RILs, derived from Mesoamerican intragene pool cross, under eight environments differing in drought stress in Africa and South America. They mapped nine QTL for 10 drought-tolerant traits on six of the 11 linkage groups, with six QTL showing significant QTL $\times$ environment interaction. The QTL for SCMR and pod partitioning traits the most stable across environments, which may be used to enhance drought adaptation in common bean. The common bean genome sequence, when it becomes available, will provide a major resource to discover genomic regions differentiating stress-responsive and non-stress-responsive genotypes, and for highlighting specific molecular-based stress responses, which will assist on the selection of parental lines to improve the efficiency of common bean improvement programs (McClean et al., 2011).

\subsubsection{Salinity Tolerance in Cereals and Legumes}

Rice, wheat and barley, among the cereal crops, have been most extensively studied to dissect the physiological and genetic basis of salt tolerance and the knowledge gained from such studies may help to develop salt-tolerant cultivars. Essentially, the salt tolerance in these crops is sodium exclusion, which limits the entry of sodium into the plants and its transport to the leaves, resulting in relatively higher $\mathrm{K}^{+} / \mathrm{Na}^{+}$ratio in the plant. Although genetic variation for salt tolerance has been reported in almost all major cereal crops, its genetic basis and genomic regions associated with salinity tolerance have been discovered only in rice, wheat and barley. Further, a major salt-tolerance QTL (SKC1) on rice chromosome 1 has been cloned, which expresses in the parenchyma cells surrounding the xylem vessels. SKC1 encodes a sodium transporter involved in regulating $\mathrm{K}^{+} / \mathrm{Na}^{+}$homoeostasis under salt stress (Dwivedi et al., 2010 and references therein). Saltol (located on chromosome 1) has been the major QTL for marker-assisted selection in rice (Thomson et al., 2010). Targeted breeding for salt tolerance led to the release of few salt-tolerant rice and wheat cultivars in salt-affected areas of Egypt, India, Indonesia, Pakistan, 
the Philippines, Thailand, the United Kingdom, and Vietnam (Dwivedi et al., 2010 and references therein).

Legumes unlike cereals have received less attention to identifying and understanding the physiological and genetic basis of salinity tolerance. Soybean is probably the most extensively studied legume crop for salinity tolerance. Sources of resistance both in cultivated soybean and its wild progenitors have been identified (Parker et al., 1983; Xu et al., 1999; Li et al., 2000; Hamwieh and $\mathrm{Xu}, 2008$ ), and the physiological basis of salt tolerance is fairly known (Luo et al., 2005; Phang et al., 2008). Essentially, salt tolerance includes maintaining ion homeostasis by withholding toxic ions from sensitive aerial parts, adjusting osmotic potential in cell by accumulating metabolites, and restoring oxidative balance to prevent further damage due to excess accumulation of reactive oxygen species (ROS). Furthermore, in some genotypes, as is the case with soybean WF 7, multiple mechanisms in part operate (Abscisic acid (ABA)-dependent pathway, involvement of ROS and withholding toxic $\mathrm{Cl}^{-}$ ions from leaves) to confer salt tolerance (Ren et al., 2012). The salt tolerance in Glycine soja (soybean progenitor) is primarily from exclusion of sodium ions preventing accumulation at the toxic concentrations in stems and leaves. This difference in tolerance mechanisms in Glycine max and G. soja indicates that interspecific crosses between these two species offer the possibility to improve salt tolerance in soybean cultivars. Furthermore, the major QTL for salt tolerance have been mapped on linkage group $\mathrm{N}$ and validated across environments and genetic populations (Lee et al., 2004, 2009; Hamwieh et al., 2011). More importantly, recent research indicates that the salt-tolerant QTL is conserved in both wild and cultivated soybeans (Hamwieh and $\mathrm{Xu}, 2008$ ). The strong relationship between the microsatellite alleles and salt tolerance suggests that these markers could be used for marker-assisted selection in soybean breeding. Very recently, salinity-tolerant germplasm in chickpea, pigeonpea and peanut were identified and they provide means for researching the physiological basis of salt tolerance in these crops. For example, reproductive stage in chickpea is most sensitive to salinity and tolerance is not related to the shoot $\mathrm{Na}^{+}$or $\mathrm{K}^{+}$ but ability to maintain a large number of filled pods under saline conditions (Vadez et al., 2007a; Krishnamurthy et al., 2011). Clearly, more such studies are needed to better understand the component traits and the genomic regions associated with these traits conferring salt tolerance in major legume crops.

\subsubsection{Biofortification to Enhancing Nutritional Quality of Food Crops}

Micronutrient malnutrition arising from $\mathrm{Fe}, \mathrm{Zn}$ and vitamin A deficiencies affects billions of people around the world (http://www.unscn.org). 
Widespread micronutrient malnutrition results in an enormous negative socioeconomic impact at the individual, community, and national levels (Darnton-Hill et al., 2005; Stein, 2010). More importantly, the rise in atmospheric $\mathrm{CO}_{2}$ and temperature, as a result of climate change and variability, has been found associated with declining nutritional value of food crops (Section 2). Crop biofortification is a sustainable and cost-effective strategy to address malnutrition in developing countries. Several reviews on crop biofortification as a strategy have been published (see Dwivedi et al. 2012 for the most recent one). Plant breeders have used available natural variations for nutritional traits for developing nutrient-dense cultivars for some cereal and legume crops. To date, a few nutrient-dense cultivars have already been released for cultivation in some regions, e.g. seed iron-dense common bean and rice in Latin America; seed iron-dense common bean in eastern and southern Africa; and seed iron-dense rice in the Philippines. There are more nutritionally enhanced lines in the release pipeline. Temperate maize germplasm have shown exceptionally large variations in $\beta$-carotene, which have been transferred into tropical maize hybrids. They are being evaluated prior to their release in Mexico and in some countries in Africa. The high $\beta$-carotene trait in Golden Rice 2 is being introgressed into several Asian rice cultivars. Molecular markers for $L \gamma c E$ and $H \gamma d B$ linked to increased $\beta$-carotene have been fully implemented in some maize breeding programs, which has accelerated breeding by one season and substantially enhance efficiency and effectiveness of high-provitamin A maize breeding. Markerassisted selection has been successfully employed to transfer low phytate into improved soybean cultivars. Enhancing nutritional quality of food crops through transgene(s) is also underway for select crops and nutrients. Biofortified crops are also being investigated for efficacy with human and animal systems. Biofortification has been included as core breeding activity in some countries in Latin America to ensure that newly developed crop cultivars meet nutritional needs of humans (Dwivedi et al., 2012 and references therein). Clearly, more efforts are needed to strengthen crop biofortification as a strategy to develop nutritionally dense cultivars for the major food crops worldwide.

\subsection{Genetically Modified Crops Tolerant to Abiotic Stresses}

Recently, Dwivedi et al. (2010) provided a detailed account of the progress realized through genetic modification for developing putative transgenic plants in barley, maize, rice and wheat, which showed an improved adaptation to drought, salinity or extreme temperature. A number of genes including 
DREB and its homolog, HVA1, ZmPLC1, Sod1, HARDY, OsNAC6, CMO, SsNHX1, and AtAVP1, to name a few, have been used to develop transgenic events with improved adaptation to drought, heat or salinity stress (Dwivedi et al., 2010). Isopentenyltransferase (IPT) is a critical enzyme in the cytokinin biosynthetic pathway. The expression of IPT under the control of a maturation- and stress-induced promoter delays stress-induced plant senescence, which results in an enhanced adaptation to drought in both monocot and dicot plants (Rivero et al., 2007, 2009, 2010). The transgenic rice plants expressing $P_{S A R K}: I P T$ gene showed enhanced drought adaptation and significantly higher grain yield with improved quality (nutrients and starch content) when compared to wild types (Peleg et al., 2011).

Pellegrineschi et al. (2004) were probably the first to generate and evaluate in a greenhouse $D R E B 1 A$-wheat transgenic events with enhanced survival to severe drought stress at the 4-5 leaf stage. The selected transgenic wheat lines showed water stress symptoms later than the control. More recently, Saint Pierre et al. (2012) evaluated 14 of these selected transgenic lines (five with high WUE, WUE-11 to WUE-14, and nine that survived severe water-stress conditions in previous evaluation) for biomass and WUE under greenhouse conditions and for survival to severe water deficit in the field conditions. Their results revealed that AtDREB1A driven by the stressinducible promoter rd29A increased the survival rate of transgenic plants without growth retardation. They also note a positive association between WUE and biomass, which suggests that an increase in grain yield may be possible by increasing WUE in transgenic plants (assuming that the HI is maintained). The selected events, due to their WUE in greenhouse screens, were found to combine an acceptable yield—and even higher yield for WUE11 under well-watered condition-and stable performance, without any pleiotropic effects, across the environments in their field experiment. Taken together, it is encouraging to be optimistic that the goal of highyielding wheat transgenic lines would be achievable in near future if adequate transformation and screening protocols are implemented.

Bhatnagar-Mathur et al. (2007, 2009a,b) introduced P5CSF129A in chickpea and $D R E B 1 A$ in peanut. Both are driven by the stress-inducible promoter $r d 29 A$. The transgenic plants overexpressing P5CSF129A or DREB1A showed substantial increase in TE in the greenhouse (Bhatnagar-Mathur et al. 2009a,b). Root traits are an important source of variation and provide mechanisms to enhance adaptation to drought in plants. Vadez et al. (2008) found that transgenic plants overexpressing DREB1A had enhanced root growth under water-deficit conditions, 
particularly in the deep soil layers. Some transgenic events had enhanced water uptake under water-deficit conditions up to 20-30\% compared with wild types, and they were highly correlated $\left(r^{2}=0.91\right)$ with root dry weight below the $40 \mathrm{~cm}$ soil depth in the profile (Vadez et al., 2007b). Jyostna Devi et al. (2009b) noted that high TE in some transgenic events, containing $r d 29 A$ :DREB1A construct, under drought-stressed conditions were significantly correlated with SCMR. Furthermore, they detected a significant negative correlation between TE and FTSW threshold values where transpiration declines upon soil drying, which led to higher TE.

The regulation of $P_{S A R K}: I P T$ in peanut significantly improved drought tolerance under both laboratory and field conditions. The transgenic plants did not only maintain higher photosynthetic rates, higher stomatal conductance, and higher transpiration than wild-type (WT) plants under reduced irrigation conditions, but also produced significantly higher yield (58\% more seeds on average based on 2 years data) than WT plants in the field, indicating a great potential for the development of crops with improved performance and yield in the water-limited areas of the world (Qin et al., 2011).

Tissue tolerance (to accumulate $\mathrm{Na}^{+}$or $\mathrm{Cl}^{-}$) is an important adaptive mechanism in plants to salinity (Munns and Tester, 2008). Membrane proteins, such as vacuole $\mathrm{Na}^{+} / \mathrm{H}^{+}$antiporters (NHX), play key roles in tissue tolerance to $\mathrm{Na}^{+}$in vacuoles in plants (Apse et al., 1999; Blumwald et al., 2000). For example, overexpression of OsNHX1 and TaNHX 2 in rice and wheat, respectively, confers high salt tolerance to transgenic plants (Chen et al., 2007; Jian et al., 2009). Recently, Cao et al. (2011) transformed a TaNHX 2 gene into soybean and the homozygous transgenic lines overexpressing TaNHX 2 showed enhanced salt tolerance, as measured by plant biomass and flowers plant ${ }^{-1}$, compared to WT plants grown on sand culture containing $150 \mathrm{mM} \mathrm{NaCl}$. The transgenic line, C12-11 showed longer survival, less growth inhibition and greater number of flowers than wild types, indicating that overexpression of TaNHX 2 could enhance salt tolerance in soybean.

In summary, crop germplasm enhancement supports applied plant breeding in the form of providing intermediate genetic materials with beneficial traits for cultivar development. CWR contributed many beneficial alleles to broaden the genetic base of food crops. Resynthesized lines contributed rich source of variation for enhancing resistance to abiotic and biotic stresses in wheat.These amphiploids are expected to release new source of variation for use in genetic improvement of peanut. There is increasing emphasis to develop marker-aided introgression of novel alleles from exotic germplasm onto the 
background of elite crop cultivars to enhance the trait value for use in crop improvement. Sources of resistance and genomic regions (QTLs) associated with abiotic stresses have been identified, both in cereal and legume crops. Few QTLs with major effect for drought, salinity and submergence tolerance have been mapped or cloned. The physiological basis of drought tolerance has been dissected into component traits such as drought escape (early maturity), drought avoidance root traits, stay-green QTL, and conservative use of water under high VPD as an important adaptive strategy for adaptation to drought environments; all these traits have potential to design plant ideotypes from specific allele combinations conferring particular physiological traits for specific adaptation to a range of terminal drought conditions. A major QTL for submergence (Sub1) tolerance has been introgressed and the products of such introgressions have been recently released for cultivation in some Asian countries. The major QTL for phosphorus deficiency (Pup1) has been the target for the development of tolerant rice cultivars. The NILs containing Pup 1, mostly conserved in rice germplasm and cultivars adapted to droughtprone environments, conferred significant yield advantage in P-deficient soils. Likewise, a major QTL for salt tolerance (Saltol) is being introgressed into rice cultivars. Targeted breeding for drought and salinity tolerance has led to the development and release of several cultivars resistance to these stresses in chickpea, common bean, cowpea, maize, peanut, pearl millet, rice, sorghum, and soybean in some African and Asian countries. A number of genes including $D R E B$ and its homologs have been used to develop transgenic events with improved adaptation to drought, heat or salinity stress in chickpea, maize, peanut, rice, and wheat, with many events showing no adverse impact on agronomic traits. Global warming is also associated with declining nutritional quality of food crops (see Section 2). Plant breeders have also succeeded in developing nutrient-dense cultivars in common bean, maize, and, rice that have been released in some countries, while many nutritionally enhanced lines in the release pipeline. The high $\beta$-carotene trait in "Golden Rice 2" is being introgressed into several Asian rice cultivars. Enhancing nutritional quality of food crops through transgene(s) is also underway, particularly for $\mathrm{Fe}$ and $\beta$-carotene in rice grains.

\section{OUTLOOK}

The information available especially from the IPCC reports, and climate models predict that some regions of the world, especially in developing world, will be greatly adversely affected due to global warming, thereby 
poising of threat to both food production and quality for human nutrition and feed for livestock. The crop produce could be likely less nutritious, thereby spreading more malnutrition in the developing world. Research to-date suggests that drought, heat and elevated $\mathrm{CO}_{2}$ reduce food and feed quality. However, genotypic differences with respect to grain-quality attribute under drought and heat stress do provide researchers opportunities to identify crop germplasm or cultivars with lowest differences in grain quality under stress. Such germplasm are the ideal sources for use in crop improvement programs, and to breed cultivars with no adverse effect of global warming on grain quality. To support the development of nutritious crops, a number of novel high-throughput assays are now available to facilitate screening of a large numbers of grain samples for seed composition and quality. The feasibility for breeding seed-nutrient-dense biofortified crops has been shown in crops such as bean, maize, pearl millet, rice, and wheat (Dwivedi et al., 2012). However, a paradigm shift is needed to include biofortification in core breeding programs to assure that no crop cultivars that do not meet the minimum quality attributes are released for cultivation. Another important research area will be to assess how global warming may affect the bioavailability of plant nutrients?

The threat to altered pest and disease dynamics will adversely impact agricultural production, while increased risk of mycotoxin contamination will make the produce unsafe for human and livestock consumption. The current knowledge about the impact of global warming on pests and pathogens affecting agricultural crops is highly limited. We call upon researchers to generate more empirical data on the host-pathogen or host-insect biology under emerging climates as the emergence of more aggressive pathotypes (or biotypes) may reduce effectiveness of host-plant resistances, leading to substantial loss to production. There is need to develop crop-pest (pathogen) models that predict potential geographic distribution, seasonal phenology and population dynamics at a range of spatial and temporal scale, which will provide researchers opportunity to design sound plant health management practices for pathogens and pests.

The use of geostatistics, GIS and satellite imagery data are adding to our knowledge to predict and monitor shifts in community structure of the aflatoxin-producing fungi $A$. flavus. Limited studies on use of long-term weather and crop yields data together with crop models have made it possible to predict the risk of aflatoxin contamination in agricultural produce. The NARCCAP database (www.narcap.ucar.edu), which provides ranges of climatic conditions, offers opportunities to assess how future climate 
scenarios may affect aflatoxin levels around the world. Cost-effective assays such as cELISA and Afla-ELISA are providing unique opportunity to evaluate food, feed and related commodities for aflatoxin contamination. The water activity $\left(a_{\mathrm{w}}\right)$, temperature, and their interactions with aflatoxin gene cluster significantly impact the fungal growth and toxin production. The mycotoxin biosynthesis gene-specific microarray provides researchers opportunities to identify food-borne fungi, the most common mycotoxins, and investigate the influence of environmental parameters and their interactions to predict and identify mycotoxin biosynthesis genes, and adopt a system-based approach to control mycotoxin contamination in food crops. Atoxigenic strains of $A$. flavus can be used as biopesticide because they provide an effective control and minimize risk of aflatoxin contamination in cotton, maize and peanut. Efforts should be directed toward identifying locally adapted atoxigenic strains competitive enough to replace naturally occurring toxigenic strains. The use of the biocontrol agent "Aflasafe" (based on an atoxigenic strain) is gaining momentum and large-scale adoption trials are underway to assess its efficacy to minimize aflatoxin contamination in maize in Nigeria. This approach is also being experimented using locally adapted atoxigenic strains to control aflatoxin contamination in maize in Kenya and the Republic of Benin. We envisage that this biocontrol approach will find wider acceptance and together with resistant cultivars and other management options will provide effective measures to minimize the risk of aflatoxin from agricultural produce in developing world.

Agrobiodiversity is crucial to coping with adverse impact of global warming on food production and quality. The most threatened plants are CWR, and every effort should be made for their in situ conservation in protected areas to ensure in situ evolutionary process of wild species contributing new variants, which contribute to addressing new challenges to agricultural production. Taping new variants for phenological traits as a result of global warming is another window of opportunity to address for life-history traits for changes in frequency of alleles favoring adaptation to global warming, as evidenced for shift in early flowering alleles at PHYC locus and reduction in plant and spike size in pearl millet in Sahel (Vigouroux et al., 2011). It is understood that soil fertility will be greatly impacted by increase in both atmospheric $\mathrm{CO}_{2}$ concentration and temperature. While increase in $\mathrm{CO}_{2}$ concentration will mean opportunity for enhanced photosynthetic rate, the growing plants will not be able to take advantage of this unless the photosynthesis-driven increased nutrient requirement is met through balanced and integrated nutrient management strategy. Soil microbial population, 
especially microbial structure diversity will play a critical role in this effort. Increased temperature will influence soil fertility and nutrient availability and uptake by its influence on soil water (would decrease soil water content and duration) and organic matter decomposition (decomposition rate would increase provided soil moisture is available).

The genetic base of many of our food crops is narrow because of the bottlenecks associated with domestication of these species. Germplasm enhancement contributes to broadening the total genetic diversity in crops, and provides specific traits to plant breeders. The evidence to-date in peanut, rice, tomato and wheat suggests that CWR (that could be the main source of genetic variation for some crops) often provide alleles that enhance the trait value in crop cultigens. It is suggested that CWR and other exotic germplasm should be integrated in breeding programs to develop intermediate breeding products such as introgression lines with specific characteristics, not present in cultigens gene pool, to support crops breeding. The crop genetic enhancers to-date have made considerable progress toward identifying sources of resistance and genomic regions (QTLs) associated with abiotic stresses, that when transferred into improved genetic background contributed to enhance adaptation of newly developed germplasm to these stresses. Targeted breeding (including use of applied genomic tools) for enhanced adaptation has led to the development and release of cultivars of chickpea, common bean, cowpea, maize, peanut, pearl millet, rice, sorghum, and soybean with enhanced adaptation to drought, salinity and flooding in some African and Asian countries. Furthermore, plant response to these abiotic stresses in many cases has been dissected at physiological scale, and many component traits contributing enhanced adaptation have been identified, which plant breeders are using to develop new germplasm with specific characteristics. Use of transgene(s) to support conventional breeding has led to the development of transgenic events with improved adaptation to abiotic stresses, in some cases with no adverse effects on agronomic traits in chickpea, maize, peanut, rice and wheat. These transgenic events are in various stages of evaluation and characterization and when released will be a valuable resource either as cultivars or use as source materials to transfer these transgene(s) into locally adapted cultivars enhancing their adaptation to abiotic stresses.

Climate change analogs today provide a view of tomorrow's agriculture. They offer a virtual natural laboratory to innovate and test various technological options to develop climate resilience production systems for large-scale adoption by the farming community. The available information generated through this approach may also provide researchers an 
opportunity to establish "atlas of climate sensitivities" for multiple crops in various regions to assist growers adopt a"cafeteria approach"to mitigate the adverse effects of climate change and variability.

Plant phenomics offers a suit of new technologies for accelerated progress in breeding crop germplasm with specific attributes, and to diagnose nutrient and environmental stresses to adapt appropriate crop management options to ameliorate these stresses in commercial agriculture.Various automatic high-throughput phenotyping platforms are available to accurately measure plant growth and development. The response to abiotic stresses can be dissected at physiological levels that will facilitate to accurately determine its genetic control, thus, providing new opportunities to enhance our knowledge of plant response to abiotic stresses, which may help develop improved germplasm with specific attributes. Such a rapid development in high-throughput assays allows researchers phenotype and genotype large sets of individuals. Linking this large data is a major issue for basic and applied genomic-centric biological research. Developing an appropriate platform that efficiently stores, adds, retrieves, and or modifies, queries it, and analyzes these datasets is needed; and use of "ChadoNatural Diversity" module (http://gmod.org/wiki/Chado_Natural_Diversity_Module) is recommended as it has inbuilt mechanisms to store large-scale phenotyping, genotyping and breeding data.

It is evident from the forgoing discussion that no one solution will suffice to fight climate change and variability effects. Suits of technological innovations including nutritionally enhanced climate-resilient crop cultivars will be needed to fight adverse impact of climate change variability on rural peasant lives, which will help produce food to feed the 9 billion mouths.

\section{ACKNOWLEDGMENTS}

We appreciate H.C. Sharma of ICRISAT for his critical review of the manuscript and ICRISAT library personnel for arranging reprints. Sangam Dwivedi highly appreciates the support and encouragement from Dr William D. Dar (director general, ICRISAT).

\section{REFERENCES}

Abbas, H.K., Weaver, M.A., Horn, B.W., Carbone, I., Monacell, J.T., Shier, W.T., 2011a. Selection of Aspergillus flavus isolates for biological control of aflatoxins in corn. Toxin. Rev. 30, 59-70.

Abbas, H.K., Zablotowicz, R.M., Horn, B.W., Phillips, N.A., Johnson, B.J., Jin, X., Abel, C.A., 2011b. Comparison of major biocontrol strains of non-aflatoxigenic Aspergillus flavus for the reduction of aflatoxins and cyclopiazonic acid in maize. Food Addit. Contam. 28, 198-208. 
Abbas, H.K., Zablotowicz, R.M., Abel, C.A., 2006. Biocontrol of aflatoxin in corn by inoculation with non-aflatoxigenic Aspergillus flavus isolates. Biocontrol Sci. Technol. 16, 439-449.

Abbas, H.K., Zablotowicz, R.M., Accinelli, C., Abel, C.A., Bruns, H.A., Weaver, M.A., 2009. Ecology of Aspergillus flavus, regulation of aflatoxin production, and management strategies to reduce contamination of corn. Toxin. Rev. 28, 142-153.

Abbate, P.E., Dardanelli, J.L., Cantarero, M.G., Maturano, M., Melchiori, R.J.M., Suero, E.E., 2004. Climate and water availability effects on water-use efficiency in wheat. Crop Sci. 44, 474-483.

Abdel-Hadi, A., Carter, D., Magan, N., 2010. Temporal monitoring of the nor-1 (aflaD) gene of Aspergillus flavus in relation to aflatoxin $\mathrm{B}_{1}$ production during storage of peanuts under different water activity levels. J. Appl. Microbiol. 109, 1914-1922.

Abdel-Hadi, A., Schmidt-Heydt, M., Parra, R., Geisen, R., Magan, N., 2011. A systems approach to model the relationship between aflatoxin gene cluster expression, environmental factors, growth and toxin production by Aspergillus flavus. J. R. Soc. Interface. http://dx.doi.org/10.1098/rsif.2011.0482.

Acosta-Diaz, E., Acosta-Gallegos, J.A., Trejo-López, C., Padilla-Ramírez, J.S., AmadorRamírez, M.D., 2009. Adaptation traits in dry bean cultivars grown under drought stress. Agricultura Técnica en México 35, 416-425.

Acosta-Gallegos,J.A., Adams, M.W., 1991. Plant traits and yield stability of dry bean (Phaseolus vulgaris) cultivars. J. Agric. Sci. (Cambridge) 117, 213-219.

Acosta-Gallegos, J.A., Kelly, J.D., Gepts, P., 2007. Prebreeding in common bean and use of genetic diversity from wild germplasm. Crop Sci. 47 (S3), S44-S59.

Acosta-Gallegos, J.A., Kohashi-Sibata, J., 1989. Effect of water stress on growth and yield of indeterminate dry bean (Phaseolus vulgaris) cultivars. Field Crops Res. 20, 81-90.

Acosta-Gallegos, J.A., White, J.W., 1995. Phenological plasticity as an adaptation by common bean to rainfed environments. Crop Sci. 35, 199-204.

Ahmadi, M., Bahrani, M.J., 2009. Yield and yield components of rapeseed as influenced by water stress at different growth stages and nitrogen levels. America-Eurasian J. Agric. Environ. 5, 755-761.

Ainsworth, E.A., Long, S.P., 2005. What have we learned from 15 years of free-air $\mathrm{CO}_{2}$ enrichment (FACE)? A meta-analytic review of the responses of photosynthesis, canopy properties and plant production to rising $\mathrm{CO}_{2}$. New Phytol. 165, 351-372.

Ainsworth, E.A., Ort, D.R., 2010. How do we improve crop production in a warming world? Plant Physiol. 154, 526-530.

Akin, D.E., Kimball, B.A., Windham, W.R., Pinter Jr., P.J., Wall, G.W., Garcia, R.L., LaMorte, R.L., Morrison III, W.H., 1995. Effect of free-air $\mathrm{CO}_{2}$ enrichment (FACE) on forage quality of wheat. Anim. Feed Sci. Technol. 53, 29-43.

Ali, Q., Ashraf, M., Anwar, F., 2010. Seed composition and seed oil antioxidant activity of maize under water stress. J. Am. Oil Chem. Soc. 87, 1179-1187.

Ali, Q., Muhammad, A., Farooq, A., 2009. Physico-chemical attributes of seed oil from drought stressed sunflower (Helianthus annuus L.) plants. Grasas Y Aceites 60, 475-481.

Al-Suhaibani, N.A., 2009. Influence of early water deficit on seed yield and quality of faba bean under arid environment of Saudi Arabia. America-Eurasian J. Agric. Environ. 5, 649-654.

Altieri, M.A., 1999. The ecological role of biodiversity in agroecosystems. Agric. Ecosyst. Environ. 74, 19-31.

Apse, M.P., Ahron, G.S., Snedden, W.A., Blumwald, E., 1999. Salt tolerance conferred by overexpression of a vacuolar $\mathrm{Na}^{+} / \mathrm{H}^{+}$antiport in Arabidopsis. Science 285, 1256-1258.

Araus, J.L., Casadesus, J., Bort, J., 2001. Recent tools for the screening of physiological traits determining yield. In: Reynolds, M.P., Ortiz-Monasterio, J.I., McNab,A. (Eds.), Application of Physiology in Wheat Breeding, CIMMYT, Mexico, D.F, pp. 59-77. 
Asfaw, A., Almekinders, C.J.M., Blair, M.W., Struik, P.C., 2012a. Participatory approach in common bean (Phaseolus vulgaris L.) breeding for drought tolerance for southern Ethiopia. Plant Breed. 131, 125-134.

Asfaw, A., Blair, M.W., Struik, P.C., 2012b. Multienvironment quantitative trait loci analysis for photosynthate acquisition, accumulation, and remobilization traits in common bean under drought stress. G3 Genes Genomes Genetics 2, 579-595.

Asseng, S., Foster, I., Turner, N.C., 2011. The impact of temperature variability on wheat yields. Glob. Change Biol. 17, 997-1012.

Atehnkeng, J.P., Cotty, P.J., Bandyopadhyay, R., 2010. Mitigation of aflatoxin contamination in Nigerian maize with atoxigenic strain mixtures. Phytopathology 100, S8 (abstract).

Atehnkeng, J., Ojiambo, P.S., Ikotun, T., Sikora, R.A., Cotty, P.J., Bandyopadhyay, R., 2008. Evaluation of atoxigenic isolates of Aspergillus flavus as potential biocontrol agents for aflatoxin in maize. Food Addit. Contam. 25, 1266-1273.

Baianu, I.C., You, T., Costescu, D.M., Lozano, P.R., Prisecaru, V., Nelson, R.L., 2012. Determination of soybean oil, protein and amino acid residues in soybean seeds by high resolution nuclear magnetic resonance (NMRS) and near infrared (NIRS). Nat. Proc. http://dx.doi.org/10.1038/npre.2012.7053.1.

Bailey-Serres, J., Fukao, T., Ronald, P., Ismail, A., Heuer, S., Mackill, D., 2010. Submergence tolerant rice: SUB1's journey from landrace to modern cultivar. Rice 3, 138-147.

Balla, K., Rakszegi, M., Li, Z., Békés, F., Bencze, S.,Veisz, O., 2011. Quality of winter wheat in relation to heat and drought shock after anthesis. Czech J. Food Sci. 29, 117-128.

Balla, K., Rakszegi, M., Bencze, S., Karsai, I., Veisz, O., 2010. Effect of high temperature and drought on the composition of gluten proteins in Martonvásár wheat varieties. Acta Agronomica Hungarica 58, 343-353.

Bänziger, M., Edmeades, G.O., Lafitte, H.R., 1999. Selection for drought tolerance increases maize yields across a range of nitrogen levels. Crop Sci. 39, 1035-1040.

Barbehenn, R.V., Chen, Z., Karowe, D.N., Spickard, A., 2004. C 3 grasses have higher nutritional quality than $\mathrm{C}_{4}$ grasses under ambient and elevated atmospheric $\mathrm{CO}_{2}$. Glob. Change Biol. 10, 1565-1575.

Barber, S.A., 1995. Soil Nutrient Bioavailability: A Mechanistic Approach, second ed. Wiley, New York, NY, USA.

Barbetti, M.J., Banga, S.S., Salisbury, P.A., 2012. Challenges for crop production and management from pathogen biodiversity and diseases under current and future climate scenarios - case study with oilseed Brassicas. Field Crops Res. 127, 225-240.

Bardgett, R.D., 2011. Plant-soil interactions in a changing world. FlOOO Rep. Biol. 3, 16.

Bardgett, R.D., Usher, M.B., Hopkins, D.W. (Eds.), 2005. Biological Diversity and Functions in Soils, Cambridge University Press, Cambridge, UK.

Barea, J.-M., Pozo, M.J., Azcon, R., Azcon-Aguillar, C., 2005. Microbial co-operation in the rhizosphere. J. Exp. Bot. 56, 1761-1778.

Baring, M.R., Simpson, C.E., Burow, M.D., Cason, J.M., Ayers, J.L., 2012. Registration of Tamrun OL11 peanut. J. Plant Registrations. 7, 1-5.

Barrios, E., 2007. Soil biota, ecosystem services and land productivity. Ecol. Econ. 64, 269-285.

Bassirirad, H., 2000. Kinetics of nutrient uptake by roots: responses to global change. New Phytol. 147, 155-169.

Battilani, P., Barbano, C., Piva, G., 2008. Aflatoxin B 1 contamination in maize related to the aridity index in North Italy. World Mycotoxin J. 1, 449-456.

Battisti, D.S., Naylor, R.L., 2009. Historical warnings of future food insecurity with unprecedented seasonal heat. Science 323, 240-244.

Bauriegel, E., Giebel, A., Herppich, W.B., 2011. Hyperspectral and chlorophyll fluorescence imaging to analyze the impact of Fusarium culmorumon the photosynthetic integrity of infected wheat ears. Sensors 11, 3765-3779. 
Beebe, S., Rao, I.M., Cajiao, C., Grajales, M., 2008. Selection for drought resistance in common bean also improves yield in phosphorus limited and favorable environments. Crop Sci. 48, 582-592.

Beebe, S., Skroch, P.W., Tohme, J., Duque, M.C., Pedraza, F., Nienhuis, J., 2000. Structure of genetic diversity among common bean landraces of Mesoamerican origin based on correspondence analysis of RAPD. Crop Sci. 40, 264-273.

Belko, N., Zaman-Allah, M., Cisse, N., Diop, N.D., Zombre, G., Ehler, J.D., Vadez,V., 2012. Lower soil moisture threshold for transpiration decline under water deficit correlates with lower canopy conductance and higher transpiration efficiency in drought tolerant cowpea. Funct. Plant Biol. http://dx.doi.org/10.1007/FM11282.

Bell, G., Collins, S., 2008. Adaptation, extinction and global change. Evol. Appl. 1, 3-16.

Berger, B., Parent, B., Tester, M., 2010. High-throughput shoot imaging to study drought responses. J. Exp. Bot. 61, 3519-3528.

Bernier, J., Kumar, A., Venuprasad, R., Spaner, D., Atlin, G., 2007. A large-effect QTL for grain yield under reproductive stage drought stress in upland rice. Crop Sci. 47, 507-518.

Bernier, J., Kumar, A., Venuprasad, R., Spaner, D., Verulkar, S., Mandal, N.P., Sinha, P.K., Peeraju, P., Dongre, P.R., Mahto, R.N., Atlin, G., 2009. Characterization of the effect of a QTL for drought resistance in rice, qtl12.1, over a range of environments in the Philippines and eastern India. Euphytica 166, 207-217.

Bhatnagar-Mathur, P., Jyotsna Devi, M.,Vadez,V., Sharma, K.K., 2009a. Differential antioxidative responses in transgenic peanut bear no relationship to their superior transpiration efficiency under drought stress. J. Plant Physiol. 166, 1207-1217.

Bhatnagar-Mathur, P., Jyotsna Devi, M., Vadez, V., Varma, D.P.S., Sharma, K.K., 2009b. Over expression of Vigna P5CSF129A gene in chickpea for enhancing drought tolerance. Mol. Breed. 23, 591-606.

Bhatnagar-Mathur, P., Vadez, V., Sharma, K.K., 2007. Stress-inducible expression of Arabidopsis thaliana DREB1A in transgenic peanut (Arachis hypogaea L.) increases transpiration efficiency under water-limiting conditions. Plant Cell Rep. 26, 2071-2082.

Biggs, T.W., Mishra, P.K., Turral, H., 2008. Evapo-transpiration and regional probabilities of soil moisture stress in rainfed crops, southern India. Agric. For. Meteor. 148, 1585-1597.

Blair, M.W., Galeano, C.H., Tovar, E., Torres, M.C.M., Castrillón, A.V., Beebe, S.E., Rao, I.M., 2012. Development of a Mesoamerican intra-genepool genetic map for quantitative trait loci detection in a drought tolerant $\times$ susceptible common bean (Phaseolus vulgaris L.) cross. Mol. Breed. 29, 71-88.

Blankinship, J.C., Niklaus, P.A., Hungate, B.A., 2011. A meta-analysis of responses to soil biota to global change. Oceologia 165, 553-556.

Bloem, M.W., Wedel, M., Egger, R.J., 1989. Iron metabolism and vitamin A deficiency in children in northeast Thailand. Am. J. Clin. Nutr. 60, 443-454.

Bluementhal, C., Rawson, H.M., McKenzie, E., Gras, P.W., Barlow, E.W.R., Wrigley, C.W., 1996. Changes in wheat grain quality due to doubling the level of atmospheric $\mathrm{CO}_{2}$. Cereal Chem. 73, 762-766.

Blumwald, E., Ahron, G.S., Apse, M.P., 2000. Sodium transport in plant cells. Biochim. Biophys. Acta 1465, 140-151.

Boken,V.K., Hoogenboom, G., Williams, J.H., Diarra, B., Dione, S., Easson, G.L., 2008. Monitoring peanut contamination in Mali (Africa) using the AVHRR satellite data and a crop simulation model. Int. J. Remote Sens. 29, 117-129.

Boot, K.J., Allen Jr., L.H., Prasad, P.V., Baker, J.T., Gesch, R.W., Snyder, A.M., Pan, D., Thomas, M.G., 2005. Elevated temperature and $\mathrm{CO}_{2}$ impacts on pollination, reproductive growth, and yield of several globally important crops. J. Agric. Meteor. (Japan) 60, 469-474. 
Boote, K.J., Allen Jr., L.H., Prasad, P.V., Jones, J.W., 2010. Testing effects of climate change in crop models. In: Hillel, D., Rosenzweig, C. (Eds.), Handbook of Climate Change and Agroecosystems, Imperial College Press, London, UK, pp. 109-129.

Bouis, H.E., Welch, R.M., 2010. Biofortification - a sustainable agricultural strategy for reducing micronutrient malnutrition in the global south. Crop Sci. 50, S20-S32.

Branch, W.D., Takayama, T., Chinan, M.S., 1990. Fatty acid variation among U.S. runner type peanut cultivars. J. Am. Oil Chem. Soc. 67, 591-593.

Brouder, S.M.,Volenec, J.J., 2008. Impact of climate change on crop nutrient and water use efficiencies. Physiol. Plant 133, 705-724.

Brummer, E.C., Barber,W.T., Collier, S.M., Cox, T.S., Johnson, R., Murray, S.C., Olsen, R.T., Pratt, R.C., Thro, A.M., 2011. Plant breeding for harmony between agriculture and environment. Front. Ecol. Environ. 9, 561-568.

Burkey, K.D., Booker, F.L., Pursley, W.A., Heagle, A.S., 2007. Elevated carbon dioxide and ozone effects on peanut:II. Seed yield and quality. Crop Sci. 47, 1488-1497.

Buttinger, G., 2010. Aflatoxin measurements: how HPLC methods have evolved over the last 20 years? Food Addit. Contam. 27, 1266-1272.

Cabrera-Bosquet, L., Crossa, J., von Zitzewitz, J., Serret, M.D., Araus, J.L., 2012. High throughput phenotyping and genomic selection: the frontiers of crop breeding coverage. J. Integr. Plant Biol. http://dx.doi.org/10.1111/j.1744-7909.2012.01116.x.

Campbell-Lendrum, D., Bertollini, R., Neira, M., Ebi, K., McMichael, A., 2009. Health and climate change: a road map for applied research. Lancet 373, 1663-1665.

Cao, D., Hu, W., Liu, W., Yao, W., Wu, C., Liu, X., Han, T., 2011. Overexpression of TaNHX2 enhances salt tolerance of composite and whole transgenic soybean plants. Plant Cell Tissue Organ Cult. 107, 541-552.

Cardwell, K.F., Cotty, P.J., 2002. Distribution of Aspergillus section Flavi among field soils from the four agroecological zone of the Republic of Bénin, West Africa. Plant Dis. 86, 434-439.

Carvalho, I.M.M.S., 2005. Effects of water stress on the proximate composition and mineral contents of seeds of two Lupins (Lupinus albus and Lupinus mutabilis). J. Food Qual. 28, $325-332$.

Carvalho, I.S., Chaves, M., Ricardo, C.P., 2005. Influence of water stress on the chemical composition of seeds of two lupins (Lupinus albus and L. Mutabilis). J. Agron. Crop Sci. 191, 95-98.

Carvalho, I.S., Ricardo, C.P., Chaves, M., 2004. Quality and distribution of assimilates within the whole plant of Lupinus (L.Albus and L. mutabilis) influenced by water stress. J. Agron. Crop Sci. 190, 205-210.

Castro, H.F., Classen, A.T., Austin, E.E., Norby, R.J., Schadt, C.W., 2009. Climate drivers and soil microbial communities. Appl. Environ. Microbiol. http://dx.doi.org/10.1128/ aem.02874-09.

Cavagnaro, T.R., 2008. The role of arbuscular mycorrhizas in improving plant zinc nutrition under low soil zinc concentrations: a review. Plant Soil 304, 315-325.

Cavagnaro, T.R., Dickson, S., Smith, F.A., 2010. Arbuscular mycorrhizas modify plant responses to soil zinc addition. Plant Soil 329, 307-313.

Ceccarelli, S., Grando, S., Maatougui, M., Michael, M., Slash, M., Haghparast, R., Rahmanian, M., Taheri, A., Al-Yassin, A., Benbelkacem, A., Labdia, M., Mimoun, H., Nachit, M., 2010. Plant breeding and climate change. J. Agric. Sci. 148, 627-637.

Chaerle, L., Leinonen, I., Jones, H.G., Van der Straeten, D., 2007. Monitoring and screening plant populations with combined thermal and chlorophyll fluorescence imaging. J. Exp. Bot. 58, 773-784.

Chaerle, L., Lenk, S., Leinonen, I., Jones, H.G., Van der Straeten, D., Buschmann, C., 2009. Multi- sensor plant imaging: towards the development of a stress-catalogue. Biotechnol. J. 4. http://dx.doi.org/10.1002/biot.200800242. 
Chakraborty, S., Luck, J., Hollaway, G., Fitzgerald, G., White, J., 2011. Rust-proofing wheat for a changing climate. Euphytica 179, 19-32.

Chakraborty, S., Luck, J., Hollaway, G., Freeman, A., Norton, R., Garrett, K.A., Percy, K., Hopkins, A., David, C., Karnosky, D.F., 2008. Impacts of global change on diseases of agricultural crops and forest trees. CAB Rev. Perspect. Agric.Vet. Sci. Nutr. Nat. Resour. 3,054 .

Chakraborty, S., Newton, A.C., 2011. Climate change, plant diseases and food security: an overview. Plant Pathol. 60, 2-14.

Chakraborty, S., Pangga, I.B., 2004. Plant disease and climate change. In: Gillins, M., Holmes, A. (Eds.), Plant Microbiology, BIOS Scientific, London, United Kingdom, pp. 163-180.

Chakraborty, S., Pangga, I.B., Lupton, J., Hart, L., Room, P.M., Yates, D., 2000. Production and dispersal of Colletotrichum gloeosporioides spores on Stylosanthes under elevated $\mathrm{CO}_{2}$. Environ. Pollut. 108, 381-387.

Chakraborty, S., Tiedemann,A.V., Teng, P.S., 2000. Climate change: potential impact on plant diseases. Environ. Pollut. 108, 317-326.

Challinor, A.J., Ewert, F., Arnold, S., Simelton, E., Fraser, E., 2009. Crops and climate change: progress, trends, and challenges in simulating impacts and informing adaptation. J. Exp. Bot. 60, 2775-2789.

Challinor, A.J., Wheeler, T.R., 2008. Crop yield reduction in the tropics under climate change: processes and uncertainties. Agric. For. Meteorol. 148, 343-356.

Chander, S., Guo, Y.Q., Yang, X.H., Zhang, J., Lu, X.Q., Yan, J.B., Song, T.M., Rocheford, T.R., Li, J.S., 2008a. Using molecular markers to identify two major loci controlling carotenoid contents in maize grain. Theor. Appl. Genet. 116, 223-233.

Chander, S., Meng,Y., Zhang,Y.,Yan, J., Li, J., 2008b. Comparison of nutritional traits variability in selected eighty-seven inbreds from Chinese maize (Zea mays L.) germplasm. J. Agric. Food Chem. 56, 6506-6511.

Chang, P.-K., Abbas, H.K., Weaver, M.A., Ehrlich, K.C., Scharfenstein, L.L., Cotty, P.J., 2012. Identification of genetic defects in the atoxigenic biocontrol strain Aspergillus flavusK49 reveals the presence of a competitive recombinant group in the field populations. Int. J. Food Microbiol. 154, 192-196.

Chang, P.-K., Horn, B.W., Dorner, J.W., 2005. Sequence breakpoints in the aflatoxin biosynthesis gene cluster and flanking regions in non-aflatoxigenic Aspergillus flavus isolates. Fungal Genet. Biol. 42, 914-923.

Chang, P.-K., Hua, S.S., 2007. Non-aflatoxigenic Aspergillus flavus TX9-8 competitively prevents aflatoxin accumulation by Aspergillus flavus isolates of large and small sclerotial morphotypes. Int. J. Food Microbiol. 114, 275-279.

Chapuis, R., Delluc, C., Debeuf, R., Tardieu, F., Welcker, C., 2012. Resiliences to water deficit in a phenotyping platform and in the field: how related are they in maize? Euro. J. Agron. http://dx.doi.org/10.1016/j.eja.2011.12.006.

Chauhan, Y.S., Rachupati, R.C.N., Wright, G.C., 2008a. Climate change impacts on peanut quality in the sub-tropical Australia. Proc. 6th Int. Crop Sci. Congress. Republic of Korea. http://www.intlcss.org/files/congress-proceedings/2008-papers/cs1-s2/cs1-s2-o3-yashvir-singh-chauhan.pdf.

Chauhan,Y.S., Wright, G.C., Rachupati, R.C.N., 2008b. Modelling climatic risks of aflatoxin contamination in maize. Aust. J. Exp. Agric. 48, 358-366.

Chauhan, Y.S., Wright, G.C., Rachupati, R.C.N., Holzworth, D., Broome, A., Krosch, S., Robertson, M.J., 2010. Application of a model to assess aflatoxin risk in peanuts. J. Agric. Sci. 148, 341-351.

Chen, H., An, R., Tang, J., Cui, X., Hao, F., Chen, J., Wang, X., 2007. Overexpression of a vacuolar $\mathrm{Na}^{+} / \mathrm{H}^{+}$antiporter gene improves salt tolerance in upland rice. Mol. Breed. 19, 215-225. 
Chin, J.H., Gamuyao, R., Dalid, C., Bustamam, M., Prasetiyono, J., Moeljopawiro, S., Wissuwa, M., Heuer, S., 2011. Developing rice with high yield under phosphorus deficiency: Pup1 sequence to application. Plant Physiol. 156, 1202-1216.

Chin, J.H., Lu, X., Haefele, S.M., Gamuyao, R., Ismail, A., Wissuwa, M., Heuer, S., 2010. Development and application of gene-based markers for the major rice QTL Phosphorus uptake1. Theor. Appl. Genet. 120, 1073-1086.

Ching, P.C., Barber, S.A., 1979. Evaluation of temperature effects on K uptake by corn. Agron. J. 71, 1040-1044.

Chhuneja, P., Dhaliwal, H.S., Bains, N.S., Singh, K., 2006. Aegilops kotschyi and A. tauschiias sources for higher levels of grain iron and zinc. Plant Breed. 125, 529-531.

Cisse, N., Ndiaye, M., Thiaw, S., Hall, A.E., 1995. Registration of "Mouride" cowpea. Crop Sci. 35, 1215-1216.

Cisse, N., Ndiaye, M., Thiaw, S., Hall, A.E., 1998. Registration of "Melakh" cowpea. Crop Sci. 37, 1978.

Clark, R.T., MacCurdy, B., Jung, J.K., Shaff, J.E., McCouch, S.R., Aneshansley, D.J., Kochian, L.V., 2011. Three-dimensional root phenotyping with a novel imaging and software platform. Plant Physiol. 156, 455-465.

Clark, C.M., Tilman, D., 2008. Loss of plant species after chronic low-level nitrogen deposition to prairie grasslands. Nature 451, 712-715.

Coakley, S.M., Scherm, H., Chakraborty, S., 1999. Climate change and plant disease management. Ann. Rev. Phytopathol. 37, 399-426.

Conroy, J.P., Seneweera, S., Basra, A.S., Rogers, G., Nissen-Wooller, B., 1994. Influence of rising atmospheric $\mathrm{CO}_{2}$ concentrations and temperature on growth, yield and grain quality of cereal crops. Aust. J. Plant Physiol. 21, 741-758.

Cortois, R., De Deyn, G.B., 2012. The curse of black box. Plant Soil 350, 27-33.

Cotrufo, M.F., Ineson, P., Scott, A., 1998. Elevated $\mathrm{CO}_{2}$ reduces the nitrogen concentration of plant tissue. Glob. Ch. Biol. 4, 43-54.

Cotty, P.J., 1989.Virulence and cultural characteristics of two Aspergillus flavus strains pathogenic on cotton. Phytopathology 79, 808-814.

Cotty, P.J., 1997. Aflatoxin-producing potential of communities of Aspergillus section flavi from cotton producing areas in the United States. Mycol. Res. 101, 698-704.

Cotty, P.J., Bhatnagar, D., 1994.Variability among atoxigenic Aspergillus flavus strains in ability to prevent aflatoxin contamination and production of aflatoxin biosynthetic pathways enzymes. Appl. Environ. Microbiol. 60, 2248-2251.

Cotty, P.J., Jaime-Garcia, R., 2007. Influence of climate on aflatoxin producing fungi and aflatoxin contamination. Int. J. Food Microbiol. 119, 109-115.

Covey, C., 1995. Using paleoclimates to predict future climate: how far can analogy go? Clim. Change 29, 403-407.

Coviella, C.E., Trumble, J.T., 1999. Effect of elevated atmospheric carbon dioxide on insectplant interactions. Conserv. Biol. 13, 700-712.

Covington, M.B., 2004. Omega-3 fatty acids. Am. Fam. Physician 70, 133-140.

Craine, J.M., Elmore, A.J., Olson, K.C., Tolleson, D., 2009. Climate change and cattle nutritional stress. Glob. Ch. Biol. http://dx.doi.org/10.1111/j.1365-2486.2009.02060.x.

Cramer, M.D., Hawkins, H.J., Verboom, G.A., 2009. The importance of nutrition on plant water flux. Oecologia 161, 15-24.

Craufurd, P.Q., Prasad, P.V.V., Waliyar, F., Taheri, A., 2006. Drought, pod yield, pre-harvest Aspergillus infection and aflatoxin contamination on peanut in Niger. Field Crops Res. 98, 20-29.

Criseo, G., Racco, C., Romeo, O., 2008. High genetic variability in non-aflatoxigenic A.flaus strains by using Quadruplex. Int. J. Food Microbiol. 125, 341-343.

Crowley, T.J., 1990. Are there any satisfactory geologic analogs for a future greenhouse warming? J. Clim. 3, 1282-1292. 
Crusciol, C.A.C., Arf, O., Soratto, R.P., Mateus, G.P., 2008. Grain quality of upland rice cultivars in response to cropping systems in the Brazilian tropical Savanna. Sci. Agric. (Piracicaba. Braz.) 65, 468-473.

Dai, Z., Yin, Y., Wang, Z., 2009. Starch granule size distribution from seven wheat cultivars under different water regimes. Cereal Chem. 86, 82-87.

Dakora, F.D., Keya, S.O., 1997. Contribution of legume nitrogen fixation to sustainable agriculture in Sub-Saharan Africa. Soil Biol. Biochem. 29, 809-817.

Darnton-Hill, I., Webb, P., Harvey, P.W., Hunt, J.M., Dalmiya, N., Chopra, M., Ball, M.J., Bloem, M.W., de Benoist, B., 2005. Micronutrient deficiencies and gender: social and economic costs. Am. J. Clin. Nutr. 81, 1198S-1205S.

Das, M.K., Ehrlich, K.C., Cotty, P.J., 2008. Use of pyrosequencing to quantify incidence of a specific Aspergillus flavus strain within complex fungal communities associated with commercial cotton crops. Phytopathol. 98, 282-288.

Das, K.K., Panda, D., Sarkar, R.K., Reddy, J.N., Ismail, A.M., 2009. Submergence tolerance in relation to variable flood water conditions in rice. Environ. Exp. Bot. 66, 425-434.

Davies, A.M.C., 2000. William Herschel and the discovery of near infrared. Spectrosc. Eur. $12,10-16$.

Degola, F., Berni, E., Restivo, F.M., 2011. Laboratory tests for assessing efficacy of atoxigenic Aspergillus flavus strains as biocontrol agents. Int. J. Food Microbiol. 146, 235-243.

Degola, F., Berni, E., Spotti, E., Restivo, F.M., 2009. Facing the problem of "false positives": re-assessment of a multiplex RT-PCR procedure for the diagnosis of $A$. flavus mycotoxin producers. Int. J. Food Microbiol. 129, 300-305.

de Jesus Júnior, W.C.J., Valadares Júnior, R., Cecílio, R.A., Moraes, W.B., Ribeiro do Vale, F.X., Ramos Alves, F., Paul, P.A., 2008. Worldwide geographical distribution of black Sigatoka for banana: predictions based on climate change models. Sci. Agric. (Piracicaba, Braz.) 65, 40-53.

Deng, Y., He, Z., Xu, M., Qin, Y., van Nostrand, J.D., Wu, L., Roe, B.A., Wiley, G., Hobbie, S.E., Reich, P.B., Zhou, J., 2012. Elevated carbon dioxide alters the structure of soil microbial communities. Appl. Environ. Microbiol. 78, 2991-2995.

Dermody, O., O’Neill, B.F., Zangerl, A.R., Berenbaum, M.R., DeLucia, E.H., 2008. Effects of elevated $\mathrm{CO}_{2}$ and $\mathrm{O}_{3}$ on leaf damage and insect abundance in a soybean agroecosystem. Anthropod Plant Interact. 2, 125-135.

De Smet, I., White, P.J., Bengough, A.G., Dupuy, L., Parizot, B., Casimiro, I., Heidstra, R., Laskowski, M., Lepetit, M., Hochholdinger, F., Draye, X., Zhang, H., Broadley, M.R., Péret, B., Hammond, J.P., Fukaki, H., Mooney, S., Lynch, J.P., Nacry, P., Schurr, U., Laplaze, L., Benfey, P., Beeckman, T., Bennett, M., 2012. Analyzing lateral root development: how to move forward. Plant Cell. http://dx.doi.org/10.1105/tpc.111.094292.

Dominique, G., Schmid, B., Brandl, H., 2006. Influence of plant diversity and elevated atmospheric carbon dioxide levels on belowground bacterial diversity. BMC Microbiol. 6, 68 .

Donner, M., Atehnkeng, J., Sikora, R.A., Bandyopadhyaya, R., Cotty, P.J., 2009. Distribution of Aspergillus flavus section Flavi in soils of maize fields in three agroecological zone of Nigeria. Soil Biol. Biochem. 41, 37-44.

Donner, M., Atehnkeng, J., Sikora, R.A., Bandyopadhyaya, R., Cotty, P.J., 2010. Molecular characterization of atoxigenic strains for biological control of aflatoxins in Nigeria. Food Addit.Contam. 27, 576-590.

Dorner, J.W., 2009a. Development of biocontrol technology to manage aflatoxin contamination in peanuts. Peanut Sci. 36, 60-67.

Dorner, J.W., 2009b. Biological control of aflatoxin contamination in corn using a nontoxigenic strain of Aspergillus flavus. J. Food Prot. 72, 801-804.

Dowkiw,A., Wright, G.C., Cruickshank, A., Redden, R., 2000. Indirect selection for drought resistance: a pilot study. ACIAR Food Legume Newslett. 31, 4-10. 
Dudley, J.W., 2008. From means to QTL: the Illinois long- term selection experiment as a case study in quantitative genetics. Crop Sci. 48, S20-S31.

Dutton, H.J., Lancaster, C.R., Evans, C.D., Cowan, J.C., 1951. The flavor problem of soybean oil.VIII. Linolenic acid. J. Am. Oil Chem. Soc. 28, 115-118.

Dwivedi, S.L., Crouch, J.H., Mackill, D.J., Xu,Y., Blair, M.W., Ragot, M., Upadhyaya, H.D., Ortiz, R., 2007a. The molecularization of public sector crop breeding: progress, problems and prospects. Adv. Agron. 95, 163-318.

Dwivedi, S.L., Bertioli, D.J., Crouch, J.H., Walls, J.F., Upadhyaya, H.D., Fávero, A., Moretzsohn, M., Paterson, A.H., 2007b. Peanut. In: Kole, C. (Ed.), Genetic Mapping and Molecular Breeding in Plants, vol. 2. Springer, pp. 115-152.

Dwivedi, S.L., Blair, M.W., Upadhyaya, H.D., Serraj, R., Balaji, J., Buhariwalla, H.K., Ortiz, R., Crouch, J.H., 2005. Using genomics to exploit grain legume biodiversity in crop improvement. Plant Breed. Rev. 26, 176-357.

Dwivedi, S.L., Nigam, S.N., Nageshwara Rao, R.C., Singh, U., Rao, K.V.S., 1996. Effect of drought on oil, fatty acids and protein contents of groundnut (Arachis hypogaea L.) seeds. Field Crops Res. 48, 125-133.

Dwivedi, S.L., Sahrawat, K.L., Rai, K.N., Blair, M.W., Andersson, M.S., Wolfgang, P., 2012. Nutritionally enhanced staple food crops. Plant Breed. Rev. 36, 169-291.

Dwivedi, S.L., Upadhyaya, H.D., Stalker, H.T., Blair, M.W., Bertioli, D.J., Nielen, S., Ortiz, R., 2008. Enhancing crop gene pools with beneficial traits using wild relatives. Plant Breed. Rev. 30, 179-230.

Dwivedi, S., Upadhyaya, H., Subudhi, P., Gehring, C., Bajic, V., Ortiz, R., 2010. Enhancing abiotic stress tolerance in cereals through breeding and transgenic interventions. Plant Breed. Rev. 33, 31-114.

Ehleringer, J.R., Cerling, T.E., Dearing, M.D., 2002. Atmospheric $\mathrm{CO}_{2}$ as a global change driver influencing plant-animal interactions. Integr. Comp. Biol. 42, 424-430.

Ehlers, J.D., Hall, A.E., 1998. Heat tolerance of contrasting cowpea lines in short and long days. Field Crops Res. 55, 11-21.

Ehlers, J.D., Hall, A.E., Patel, P.N., Roberts, P.A., Matthews, W.C., 2000. Registration of "California Blackeye 27" cowpea. Crop Sci. 40, 854-855.

Ehrlich, K.C., Cotty, P.J., 2004. An isolate of Aspergillus flavus used to reduce aflatoxin contamination in cotton seed has a defective polyketide synthase gene. Appl. Microbiol. Biotechnol. 65, 473-478.

Ehrlich, K.C., Montalbano, B.G., Cotty, P.J., 2007. Analysis of single nucleotide polymorphisms in three genes shows evidence for genetic isolation of certain Aspergillus flavus vegetative compatibility groups. FEMS Microbiol. Lett. 268, 231-236.

Elawad, H.O.A., Hall, A.E., 2002. Registration of "Ein El Gazal" cowpea. Crop Sci. 42, 1745-1746.

Englberger, L., Schierle, J., Marks, G.C., Fitzgerald, M.H., 2003. Micronesian banana, taro, and other foods: newly recognized sources of provitamin A and other carotenoids. J. Food Comp. Anal. 16, 3-19.

Entry, J.A., Rygiewicz, P.T., Watrud, L.S., Donnelly, P.K., 2002. Influence of adverse soil conditions on the formation and function of Arbuscular mycorrhizas. Adv. Environ. Res. 7, 123-138.

Erbs, M., Manderscheid, R., Jansen, G., Seddig, S., Pacholski, A., Weigel, H.-J., 2010. Effects of free- air $\mathrm{CO}_{2}$ enrichment and nitrogen supply on grain quality parameters and elemental composition of wheat and barley grown in a crop rotation. Agric. Ecosyst. Environ. 136, 59-68.

Eshed,Y., Zamir, D., 1994. A genomic libraries of Lycopersicon pennelli in L. esculentum: a tool for fine mapping of genes. Euphytica 79, 175-179.

Evans, N., Baierl, A., Semenov, M.A., Gladders, P., Fitt, B.D.L., 2008. Range and severity of a plant disease increased by global warming. J. R. Soc. Interface 5, 525-531. 
Fang, S., Yan, X., Liao, H., 2009. 3D reconstruction and dynamic modeling of root architecture in situ and its application to crop phosphorus research. Plant J. 60, 1096-1108.

Farahani, S.M., Caichi, M.R., Mazaheri, D., Afshari, R.T., Savaghebi, Gh, 2011. Barley grain mineral analysis as affected by different fertilizing systems and by drought stress. J. Agric. Sci. Technol. 13, 315-326.

Fedoroff, N.V., Battisti, D.S., Beachy, R.N., Cooper, P.J.M., Fischhoff, D.A., Hodges, C.N., Knauf, V.C., Lobell, D., Mazur, B.J., Molden, D., Reynolds, M.P., Ronalds, P.C., Rosegrant, M.W., Sanchez, P.A.,Vonshak, A., Zhu, J.-K., 2010. Radically rethinking agriculture for the 21st century. Science 327, 833-834.

Feil, B., 1997. The inverse yield-protein relationships in cereals: possibilities and limitations for genetically improving the grain protein yield. Trends Agron. 1, 103-119.

Fernando, N., Panozzo, J., Tausz, M., Norton, R., Fitzgerald, G., Seneweera, S., 2012. Rising atmospheric $\mathrm{CO}_{2}$ concentration affects mineral nutrient and protein concentration of wheat grain. Food Chem. http://dx.doi.org/10.1016/j.foodchem.2012.01.105/.

Fischer, G., Shah, M., Velthuzen, H., 2002. Climate Change and Agricultural Vulnerability. Report prepared under UN Institutional Contract Agreement 1113 for World Summit on Sustainable Development International Institute for Applied System Analysis, Laxenburg.

Flavel, R.J., Guppy, C.N., Tighe, M., Watt, M., McNill, A., Young, I.M., 2012. Non-destructive quantification of cereal roots in soil using high-resolution X-ray tomography. J. Exp. Bot. http://dx.doi.org/10.1093/jxb/err421.

Fletcher, A.L., Sinclair, T.R., Allen Jr., L.H., 2007. Transpiration responses to vapor pressure deficit in well watered "slow-wilting" and commercial soybean. Environ. Exp. Bot. 61, $145-151$.

Fofana, M., Cherif, M., Cone, B., Futakuchi, K., Audebert, A., 2010. Effect of water deficit at grain repining stage on rice grain quality. J. Agric. Biotechnol. Sustain. Dev. 2, 100-107.

Fokunang, C.N., Tembe-Fokunang, E.A., Tomkins, P., Barkwan, S., 2006. Global impact of mycotoxins on human and animal health management. Outlook Agric. 35, 247-253.

Fonceka, D., Tossim, H.-A., Rivallan, R.,Vignes, H., Faye, I., Ndoye, O., Moretzsohn, M.C., Bertioli, D.J., Glaszmann, J.-C., Courtois, B., Rami, J.-F., 2012. Fostered and left behind alleles in peanut: interspecific QTL mapping reveals footprints of domestication and useful variation for breeding. BMC Plant Biol. 12, 26.

Frahm, M.A., Rosas, J.C., Mayek-Pérez, N., López-Salinas, E., Acosta-Gallegos, J.A., Kelly, J.D., 2004. Breeding beans for resistance to terminal drought in the lowland tropics. Euphytica 136, 223-232.

Frankel, O.H., 1984. Genetic perspective of germplasm conservation. In: Arber, W. (Ed.), Genetic Manipulations: Impacts on Man and Society, Cambridge University Press, Cambridge, UK, pp. 161-170.

Franks, S.J., Weis, A.E., 2008. A change in climate causes rapid evolution of multiple lifehistory traits and their interactions in an annual plant. J. Evol. Biol. 21, 1321-1334.

Furbank, R., Tester, M., 2011. Phenomics - technologies to relieve the phenotyping bottleneck. Trends Plant Sci. 12, 635-644.

Garbeva, P., van Veen, J.A., van Elsas, J.D., 2004. Microbial diversity in soil: selection of microbial population by plant and soil type and implications for disease suppressiveness. Annu. Rev. Phytopathol. 42, 243-270.

Garcia, I., Mendoza, R., Pomar, M.C., 2008. Deficit and excess of soil water impact on plant growth of Lotus tenuis by affecting nutrient uptake and arbuscular mycorrhizal symbiosis. Plant Soil 304, 117-131.

Gavito, M.E., Curtis, P.S., Mikkelsen, T.N., Jakobsen, I., 2001. Interactive effects of soil temperature, atmospheric carbon dioxide and soil $\mathrm{N}$ on root development, biomass and nutrient uptake of winter wheat during vegetative growth. J. Exp. Bot. 52, 1913-1923. 
Ghini, R., Hamada, E., Bettiol, W., 2008a. Climate changes and plant diseases. Sci. Agric. (Piracicaba, Braz.) 65, 98-107.

Ghini, R., Hamada, E., Pedro Júnior, M.J., Marengo, J.A., Gonçalves, R.R.V., 2008b. Risk analysis of climate change on coffee nematodes and leaf miner in Brazil. Pesquisa Agropecuária Bras. 43, 187-194.

Ghini, R., Hamada, E., Gonçalves, R.R.V., Gasparotto, L., Pereira, J.C.R., 2007. Análise de risco das mudançasclimáticasglobaissobre a Sigatoka-negra da bananeira no Brasil. Fitopatol. Bras. 32, 197-204.

Giorni, P., Battilani, P., Pietri, A., Magan, N., 2008. Effect of water activity and $\mathrm{CO}_{2}$ levels on Aspergillus flavus growth and aflatoxin production in high moisture maize post-harvest. Int. J. Food Microbiol. 122, 108-122.

Goffman, F.D., Böhme, T., 2001. Relationships between fatty acid profile and vitamin E content in maize hybrids (Zea mays L.). J. Agric. Food Chem. 49, 4990-4994.

Goicoechea, N.,Antolin, M.C., Sanchez Diaz, M., 1997. Influence of arbuscular mycorrhizae and rhizobium on nutrient content and water relations in drought stressed alfalfa. Plant Soil 192, 261-268.

Golombek, S.D., Sridhar, R., Singh, U., 1995. Effect of soil temperature on the seed composition of three Spanish cultivars of groundnut (Arachis hypogaea L.). J. Agric. Food Chem. 43, 2067-2070.

Golzarian, M.R., Frick, R.A., Rajendran, K., Berger, B., Roy, S., Tester, M., Lun, D.S., 2011. Accurate inference of shoot biomass from high throughput images of cereal plants. Plant Methods 7 (2).

González, E.M., Galvez, L., Royuela, M., Aparicio-Tejo, P.M., Arresse-Igor, C., 2001. Insights into the regulation of nitrogen fixation in pea nodules: lessons from drought, abscisic acid and increased photoassimilate availability. Agronomie 21, 607-613.

Gorbet, D.W., Knauft, D.A., 1997. Registration of SunOleic 95R peanut. Crop Sci. 37, 1392.

Gorbet, D.W., Knauft, D.A., 2000. Registration of SunOleic 97R peanut. Crop Sci. 40, 1190-1191.

Gouda, P.K.,Varma, C.M.K., Saikumar, S., Kiran, B., Shenoy,V., Sashidhar, H.E., 2012. Direct selection for grain yield under moisture stress in Oryza sativa $c v$. IR58025B $\times$ O. meridionalis population. Crop Sci. 52, 644-653.

Gowda, V.R.P., Henry, A., Vadez,V., Shashidhar, H.E., Serraj, R., 2012. Water uptake dynamics under progressive drought stress in diverse accessions of the OryzaSNP panel of rice (Oryza sativa). Funct. Plant Biol. http://dx.doi.org/10.1071/FP12015.

Gowda,V.R.P., Henry, A., Yamauchi, A., Shashidhar, H.E., Serraj, R., 2011. Root biology and genetic improvement for drought avoidance in rice. Field Crops Res. 122, 1-13.

Graham, R.D., Knez, M., Welch, R.M., 2012. How much nutritional iron deficiency in humans globally is due to an underlying zinc deficiency? Adv. Agron. 115, 1-40.

Granier, C., Aguirrezabal, L., Chenu, L., Cookson, S.J., Dauzat, M., Hamard, P., Thioux, J.J., Rolland, G., Bouchier-Combaud, S., Lebaudy, A., Muller, B., Simonneau, T., Tardieu, F., 2006. PHENOPSIS, an automated platform for reproducible phenotyping of plant responses to soil water deficit in Arabidopsis thaliana permitted the identification of an accession with low sensitivity to soil water deficit. New Phytol. 169, 623-635.

Gregory, P.J., Johnson, S.N., Newton, A.C., Ingram, J.S.I., 2009. Integrating pests and pathogens into the climate change/food security debate. J. Exp. Bot. 60, 2827-2838.

Grossmann, G., Guo, W.-J., Ehrhardt, D.W., Frommer, W.B., Sit, R.V., Quake, S.R., Meier, M., 2011. The RootChip: an integrated microfluidic chip for plant science. Plant Cell 23, 4234-4240.

Grubisha, L.C., Cotty, P.J., 2010. Genetic isolation among sympatric vegetative compatibility groups of the aflatoxin-producing fungus Aspergillus flavus. Mol. Ecol. 19, 269-280.

Guan, Y.S., Serraj, R., Liu, S.H., Xu, J.L., Ali, J., Wang, W.S., Venus, E., Zhu, L.H., Li, Z.K., 2010. Simultaneously improving yield under drought stress and non-stress conditions: a case study of rice (Oryza sativa L.). J. Exp. Bot. 61, 4145-4156. 
Gupta, R., Gopal, R., Jat, M.L., Jat, R.K., Sidhu, H.S., Minhas, P.S., Malik, R.K., 2010. Wheat productivity in indo-gangetic plains of India during 2010: terminal heat effects and mitigation strategies. PACA Newslett. 14, 1-11.

Gur, A., Zamir, D., 2004. Unused natural variation can lift yield barriers in plant breeding. PLoS Biol. 2, 1610-1615.

Gutierrez-Rodriguez, M., Reynolds, M.P., Escalante-Estrada, J.A., Rodriguez-Gonzalez, M.T., 2004. Association between canopy reflectance indices and grain yield and physiological traits in bread wheat under drought and well-irrigated conditions. Aust. J. Agric. Res. 55, 1139-1147.

Gutierrez-Rodriguez, M., Reynolds, M.P., Raun, W.R., Stone, M.L., Klatt, A.R., 2010. Spectral water indices for assessing yield in elite bread wheat genotypes under wellirrigated, water- stressed, and high-temperature conditions. Crop Sci. 50, 197-214.

Guzmán-Maldonado, S.H.,Acosta-Gallegos, J., Paredes-López, O., 2000. Protein and mineral content of a novel collection of wild and weedy common bean (Phaseolus vulgaris L.). J. Sci. Food Agric. 80, 1874-1881.

Hacisalihoglu, G., Larib, B., Settles, A.M., 2010. Near-infrared reflectance spectroscopy predicts protein, starch and seed weight in intact seeds of common bean (Phaseolus vulgaris $\mathrm{L}$.). J.Agric. Food Chem. 58, 702-706.

Haefele, S.M., Hijmans, R.J., 2007. Soil quality in rice-based rainfed lowlands of Asia: characterization and distribution. In: Aggarwal, P.K. (Ed.), Science, Technology, and Trade for Peace and Prosperity, pp. 297-308 Proc. 26th Int. Rice Res. Conf., 9-12 Oct 2006, New Delhi, India. Los Baños (Philippines) and New Delhi (India): Int. Rice Res. Inst, Indian Council Agric. Res., and Nat. Acad. Agric. Sci.

Haggar, J., Schepp, K., 2011. Coffee and Climate Change Desk Study: Impacts of Climate Change in the Pilot Country Brazil of the Coffee \& Climate Initiative. Coffee and Climate, Hamburg, Germany 78 http://www.coffeeandclimate.org/research.html.

Hall, A.E., 1992. Breeding for heat tolerance. Plant Breed. Rev. 10, 129-168.

Hall, A.E., 2004. Breeding for adaptation to drought and heat in cowpea. Euro. J. Agron. 21, 447-454.

Hall, A.E., Cisse, N., Thiaw, S., Elwad, H.O.A., Ehlers, J.D., Ismail, A.M., Fery, R.L., Roberts, P.A., Kitch, L.W., Murdock, L.L., Boukar, O., Phillips, R.D., McWatters, K.H., 2003. Development of cowpea cultivars and germplasm by the bean/cowpea CRSP. Field Crops Res. 82, 103-134.

Hallegatte, S., Hourcade, J.-C., Ambrosi, P., 2007. Using climate analogues for assessing climate change economic impacts in urban areas. Clim. Change 82, 47-60.

Hamilton, J.G., Dermody, O.C., Aldea, M., Zangerl, A.R., Rogers, A., Berenbaum, M.R., DeLucia, E.H., 2005. Anthropogenic changes in tropospheric composition increase susceptibility of soybean to insect herbivory. Environ. Entomol. 34, 479-485.

Hamwieh, A., Tuyen, D.D., Cong, H., Benitez, E.R., Takahashi, R., Xu, D.H., 2011. Identification and validation of a major QTL for salt tolerance in soybean. Euphytica 179, 451-459.

Hamwieh, A., Xu, D., 2008. Conserved salt tolerance quantitative trait locus (QTL) in wild and cultivated soybeans. Breed. Sci. 58, 355-359.

Hansen, T.H., Laursen, K.H., Perrson, D.P., Pedas, P., Husted, S., Schjoerring, J.K., 2009. Micro-scaled high throughput digestion of plant tissue samples for multi-elemental analysis. Plant Methods 5, 12.

Harjes, C.E., Rocheford, T.R., Bai, L., Brutnell, T.P., Kandianis, C.B., Sowinski, S.G., Stapleton, A.E.,Vallabhaneni, R., Williams, M., Wurtzel, E.T., Yan, J., Buckler, E.S., 2008. Natural genetic variation in Lycopene epsilon cyclase tapped for maize biofortification. Science 319, 330-333.

Harpole, W.S., Tilman, D., 2007. Grassland species loss resulting from reduced niche dimension. Nature 446, 791-793. 
Hartmann, A., Czauderna, T., Hoffmann, R., Stein, N., Schreiber, F., 2011. HTPheno: an image analysis pipeline for high-throughput plant phenotyping. BMC Bioinf. 12, 148.

Hautier, Y., Niklaus, P.A., Hector, A., 2009. Competition for light causes plant biodiversity loss after eutrophication. Science 334, 636-638.

Haywood, A.M., Ridgwell, A., Lunt, D.J., Hill, D.J., Pound, M.J., Dowsett, H.J., Dolan, A.M., Francis, J.E., Williams, M., 2011. Are there pre-Quaternary geological analogues for a future greenhouse warming? Phil. Trans. R. Soc. A 369, 933-956.

Hegde, V.S., Mishra, S.K., 2009. Landraces of cowpea, Vigna unguiculata(L.) Walp., as potential sources of genes for unique characters in breeding. Genet. Resour. Crop Evol. 56, 615-627.

Hell, K., Mutegi, C., 2011. Aflatoxin control and prevention strategies in key crops of SubSaharan Africa. Afr. J. Microbiol. Res. 5, 459-466.

Hell, K., Mutegi, C., Pandohan, P., 2010. Aflatoxin control and prevention strategies in maize for Sub-Saharan Africa. Julius-Kühn-Archiv 425, 534-541.

Hites, R.A., Foran, J.A., Carpenter, D.O., Hamilton, M.C., Knuth, B.A., Schwager, S.J., 2004. Global assessment of organic contaminants in farmed salmon. Science 303, 226-229.

Högy, P., Fangmeier, A., 2008. Effects of elevated atmospheric $\mathrm{CO}_{2}$ on grain quality of wheat. J. Cereal Sci. 48, 580-591.

Högy, P., Wieser, H., Köler, P., Schwadorf, K., Breuer, J., Erbs, M., Weber, S., Fangmeier, A., 2009a. Does elevated atmospheric $\mathrm{CO}_{2}$ allow for sufficient wheat grain quality in the future? J. Appl. Bot. Food Qual. 82, 114-121.

Högy, P., Wieser, H., Köler, P., Schwadorf, K., Breuer, J., Franzaring, J., Muntifering, R., Fangmeier, A., 2009b. Effect of elevated $\mathrm{CO}_{2}$ on grain yield and quality of wheat: results from a 3-year free- air $\mathrm{CO}_{2}$ enrichment experiment. Plant Biol. 11 (Suppl. 1), 60-69.

Holton, M.K., Lindroth, R.L., Nordheim, E.V., 2003. Foliar quality influences tree-herbivory- parasitoid interactions: effects of elevated $\mathrm{CO}_{2}, \mathrm{O}_{3}$, and plant genotype. Oceologia 137, 233-244.

Horn, B.W., Dorner, J.W., 1999. Regional differences in production of aflatoxin $\mathrm{B}_{1}$ and cyclopiazonic acid by soil isolates of Aspergillus flavus along a transect within the Unites States. Appl. Environ. Microbiol. 65, 1444-1449.

Horn, B.W., Dorner, J.W., 2011. Evaluation of different genotypes of nontoxigenic Aspergillus flavus for their ability to reduce aflatoxin contamination in peanuts. Biocontrol Sci. Technol. 21, 865-876.

Horn, B.W., Greene, R.L., 1995. Vegetative compatibility within populations of Aspergillus flavus, A. parasiticus, and A. tamarii from a peanut field. Mycologia 87, 324-332.

Huang, C., Jha, A., Sweany, R., Derobertis, C., Damann Jr., K.E., 2011. Intraspecific aflatoxin inhibition in Aspergillus flavus is thigmoregulated, independent of vegetative compatibility group and is strain dependent. PLoS One 6, e23470.

Hulshof, P.J.M., Kosmeijer-Schuil, T., West, C.E., Hollman, P.C.H., 2007. Quick screening of maize kernels for provitamin A content. J. Food Comp. Anal. 20, 655-661.

Hurkman, W.J., McCue, K.F., Altenbach, S.B., Korn, A., Tanaka, C.K., Kothari, K.M., Johnson, E.L., Bechtel, D.B., Wilson, J.D., Anderson, O.D., DuPont, F.M., 2003. Effect of temperature on expression of genes encoding enzymes for starch biosynthesis in developing wheat endosperm. Plant Sci. 164, 873-881.

Husted, S., Persson, D.P., Laursen, K.H., Hansen, T.H., Pedas, P., Schiller, M., Hegelund, J.N., Schjoerring, J.K., 2011. Review: the role of atomic spectrometry in plant science. J. Anal. Spectrom. 26, 52-79.

Iftekharuddaula, K.M., Newaz, M.A., Salam, M.A., Ahmad, H.U., Mahbub, M.A.A., Septiningsih, E.M., Collard, B.C.Y., Sanchez, D.L., Pamplona, A.M., Mackill, D.J., 2011. Rapid and high precision marker-assisted backcrossing to introgress the SUB1 QTL into BR11, the rainfed lowland rice mega variety of Bangladesh. Euphytica 178, 83-97. 
IPCC, 2007. Climate change 2007: synthesis report, summary for policy makers. Technical report. http://www.ipcc.ch/ipccreports/ar4-syr.htm.

Ishimaru, T., Hirabayasi, H., Ida, M., Takai, T., San-Oh, Y.A., Yoshinaga, S., Ando, I., Ogawa, T., Kondo, M., 2010. A genetic resource for early-morning flowering trait of wild rice Oryza officinalis to mitigate high temperature-induced spikelet sterility at anthesis. Ann. Bot. 106, 515-520.

Islam, F.M.A., Basford, K.E., Jara, C., Redden, R.J., Beebe, S.E., 2002. Seed compositional and disease resistance differences among gene pools in cultivated common bean. Genet. Resour. Crop Evol. 49, 285-293.

Ismail, A.M., Hall, A.E., Ehlers, J.D., 2000. Delayed-leaf-senescence and heat-tolerance traits mainly are independently expressed in cowpea. Crop Sci. 40, 1049-1055.

Ismail,A.M., Heuer, S., Thomson, M.J.,Wissuwa, M., 2007. Genetic and genomic approaches to develop rice germplasm for problem soils. Plant Mol. Biol. 65, 547-570.

Iyer-Pascuzzi, A.S., Symonova, O., Mileyko, Y., Hao, Y., Belcher, H., Harer, J., Weitz, J.S., Benfey, P.N., 2010. Imaging and analysis platform for automatic phenotyping and trait ranking of plant root systems. Plant Physiol. 152, 1148-1157.

Jackson, R.L., Taunton, O.D., Morrisett, J.D., Gotto Jr., A.M., 1978. The role of dietary polyunsaturated fat in lowering blood cholesterol in man. Crit. Res. 42, 447-453.

Jaggard, K.W., Qi, A., Ober, E.S., 2010. Possible changes to arable crop yields by 2050. Phiols. Trans. R. Soc. Biol. Sci. 365, 2835-2851.

Jahnke, S., Menzel, M.I., van Dusschoten, D., Roeb, G.W., Büler, J., Minwuyelet, S., Blümler, P., Temperton, V.M., Hombach, T., Streun, M., Beer, S., Khodaverdi, M., Ziemons, K., Coenen, H.H., Schurr, U., 2009. Combined MRI-PET dissects dynamic changes in plant structures and functions. Plant J. 59, 634-644.

Jaime-Garcia, R., Cotty, P.J., 2003. Aflatoxin contamination of commercial cottonseed in South Texas. Phytopathol. 93, 1190-1200.

James, R.A., Caemmerer, S., Condon, A.G., Zwart, A.B., Munns, R., 2008. Genetic variation in tolerance to the osmotic stress component of salinity stress in durum wheat. Funct. Plant Biol. 35, 111-123.

Jarvis, A., Lane, L., Hijmas, R., 2008a. The effect of climate changes on crop wild relatives. Agric. Ecosyst. Environ. 126, 13-33.

Jarvis, F.I., Brown, A.H.D., Cuong, P.H., Collado-Panduro, L., Latournerie-Moreno, L., Gyawali, S., Tanto, T., Sawadogo, M., Marj, I., Sadiki, M., Thi-Ngoc Hue, N., Aria-Reyes, L., et al., 2008b. A global perspective of the richness and evenness of traditional cropvariety diversity maintained by farming communities. Proc. Natl. Acad. Sci. U S A 105, $5326-5331$.

Jeger, M.J., Pautasso, M., 2008. Plant disease and global change: the importance of long-term data sets. New Phytol. 177, 8-11.

Jian, B., Hou, W., Wu, C., Liu, B., Liu, W., Song, S., Bi, Y., Han, T., 2009. Agrobacterium rhizogenes-mediated transformation of super-root-derived Lotus carniculatus plants: a valuable tool for functional genomics. BMC Plant Biol. 25, 69-78.

Jiang, J., Yan, L., Ma, Z., 2009. Molecular characterization of an atoxigenic Aspergillus flavus strain AF051. Appl. Microbiol. Biotechnol. 83, 501-505.

Jones, D.L., Hodge, A., Kuzyakov,Y., 2004. Plant and mycorrhizal regulation of Rhizodeposition. New Phytol. 163, 459-480.

Jones, K.E., Patel, N.G., Levy, M.A., Storeygard, A., Balk, D., Gittleman, G.L., Daszak, P., 2008. Global trends in emerging infectious diseases. Nature 451, 990-993.

Jones, H.G., Sirraj, R., Loveys, B.R., Xiong, L., Wheaton, A., Price, A.H., 2009. Thermal infrared imaging of crop canopies for the remote diagnosis and quantification of plant responses to water stress in the field. Funct. Plant Biol. 36, 978-989.

Jordan, D.R., Hunt, C.H., Cruickshank, A.W., Borrell, A.K., Henzell, R.G., 2012. The relationship between the stay-green trait and grain yield in elite sorghum hybrids grown in a range of environments. Crop Sci. 52, 1153-1161. 
Jung, S., Menda, N., Redmond, S., Buels, R.M., Friesen, M., Bendana, Y., Sanderson, L.-A., Lapp, H., Lee, T., MacCallum, B., Bett, K.E., Cain, S., Clemens, D., Mueller, L.A., Main, D., 2011. The Chado Natural Diversity module: a new generic database schema for large-scale phenotyping and genotyping data. http://dx.doi.org/10.1093/database/bar051.

Jurado, M., Marín, P., Magan, N., González-Jaén, M.T., 2008. Relationship between solute and matric potential stress, temperature, growth and FUM1 gene expression in two Fusarium verticillioides strains from Spain. Appl. Environ. Microbiol. 74, 2032-2036.

Jyostna Devi, M., Sinclair, T.R.,Vadez,V., Krishnamurthy, L., 2009a. Peanut genotypic variation in transpiration efficiency and decreased transpiration during progressive soil drying. Field Crops Res. 114, 280-285.

Jyostna Devi, M.J., Bhatnagar-Mathur, P., Sharma, K.K., Serraj, R., Anwar, S.Y., Vadez, V., 2009b. Relationships between transpiration efficiency and its surrogate traits in the rd29A: DREB1A transgenic lines of groundnut. J. Agron. Crop Sci. http://dx.doi.org/ 10.1111/j.1439-037×.2011.00464.×.

Jyostna Devi, M., Sinclair, T.R.,Vadez,V., 2010. Genotypic variation in peanut for transpiration response to vapor pressure deficit. Crop Sci. 50, 191-196.

Kardol, P., Reynolds, W.N., Norby, R.J., Classen, A.T., 2011. Climate change effects on soil microarthropods abundance and community structure. Appl. Soil Ecol. 47, 37-44.

Kashiwagi, J., Krishnamurthy, L., Singh, S., Gaur, P.M., Upadhyaya, H.D., Panwar, J.D.S., Basu, P.S., Ito, O., Tobita, S., 2006. Relationships between transpiration efficiency and carbon isotope discrimination in chickpea (Cicer arietinum L.). SAT eJ 2.

Kashiwagi, J., Krishnamurthy, L., Upadhyaya, H.D., Gaur, P.M., 2008. Rapid screening technique for crop canopy temperature status and its relevance to drought tolerance improvement in chickpea. SAT eJ 6.

Kashiwagi, J., Krishnamurthy, L., Upadhyaya, H.D., Krishna, H., Chandra, S.,Vadez,V., Serraj, R., 2005. Genetic variability of drought-avoidance root traits in the mini-core germplasm collection of chickpea (Cicer arietinum L.). Euphytica 146, 213-222.

Kashiwagi, J., Upadhyaya, H.D., Krishnamurthy, L., 2010. Significance and genetic diversity of SPAD chlorophyll meter reading in chickpea germplasm in the semi- arid environments. J. Food Legumes 23, 99-105.

Kassahun, B., Bidinger, F.R., Hash, C.T., Kuruvinashetti, M.S., 2010. Stay-green expression in early generation sorghum [Sorghum bicolor (L.) Moench] QTL introgression lines. Euphytica 172, 351-362.

Kelly, A.E., Goulden, M.L., 2008. Rapid shifts in plant distribution with recent climate change. Proc. Natl. Acad. Sci. U S A 105, 11823-11826.

Khalil, S.E., Ismael, E.G., 2010. Growth, yield and seed quality of Lupinus termisas affected by different soil moisture levels and different ways of yeast application. J. Am. Sci. 6, 141-153.

Khlangwiset, P., Wu, F., 2010. Cost and efficacy of public health interventions to reduce aflatoxin-induced human disease. Food Addit. Contam. 27, 998-1014.

Kholová, J., Hash, C.T., Kakkera, A., Kočová, M., Vadez, V., 2010a. Constitutive water conserving mechanisms are correlated with terminal drought tolerance of pearl millet [Pennisetum glaucum (L.) R. Br.]. J. Exp. Bot. 61, 369-377.

Kholová, J., Hash, C.T., Kumar, P.L., Yadav, S.R., Kočová, M., Vadez, V., 2010b. Terminal drought tolerant pearl millet [Pennisetum glaucum (L.) R. Br.] have high leaf ABA and limit transpiration at high vapor pressure deficit. J. Exp. Bot. 61, 1431-1440.

Kholová, J., Napolean, T., Hash, C.T., Supriya, A., Rajaram, V., senthilvel, S., Kakkera, A., Yadav, R., Vadez, V., 2012. Water-saving traits co-map with a major terminal drought tolerance quantitative trait locus in pearl millet [Pennisetum glaucum (L.) R. Br.]. Mol. Breed. http://dx.doi.org/10.1007/s11032-012-9720-0.

Kibblewhite, M.G., Ritz, K., Swift, M.J., 2008. Soil health in agricultural systems. Phil.Trans. R. Soc. B. 363, 685-701. 
Kijne, J.W., 2004. Abiotic stress and water scarcity: identifying and resolving conflicts from plant level to global level. Field Crops Res. 97, 3-18.

Kim, K.S., Park, S.H., Choung, M.G., Jang,Y.S., 2007. Use of near-infrared spectroscopy for estimating fatty acid composition in intact seeds of rapeseed. J. Crop Sci. Biotechnol. 10, 15-20.

Kirnak, H., Dogan, E., Turkoglu, H., 2010. Effect of drip irrigation intensity on soybean seed yield and quality in the semi-arid Harran plain, Turkey. Spanish J. Agric. Res. 8, $1208-1217$.

Klueken, A.M., Borgemeister, C., Hau, B., 2009. Field release of a non-toxigenic Aspergillus flavus L strain in central Benin. J. Plant Dis. Prot. 116, 17-22.

Kobayashi, T., Ishiguro, K., Nakajima, T., Kim, H.Y., Okada, M., Kobayashi, K., 2006. Effects of elevated atmospheric $\mathrm{CO}_{2}$ concentration on the infection of rice blast and sheath blight. Phytopathol. 96, 425-431.

Kogan, F., Salazae, L., Roytman, L., 2012. Forecasting crop production using satellite-based vegetation health indices in Kansas, USA. Int. J. Remote Sens. 33, 2798-2814.

Kopf, S., Ha-Duong, M., Hallegatte, S., 2008. Using of maps of city analogues to display and interpret climate change scenarios and their uncertainty. Nat. Hazards Earth Syst. Sci. $8,905-918$.

Kotschi, J., 2007. Agricultural biodiversity is essential for adapting to climate change. GAIA 16, 98-101. www.oekom.de/gaia.

Kovalenko, L., Rippke, G., Hurburgh, C., 2006. Determination of amino acid composition of soybeans (Glycin max) by near-infrared spectroscopy. J. Agric. Food Chem. 54, 3485-3491.

Krishnamurthy, L., Kashiwagi, J., Gaur, P.M., Upadhyaya, H.D., Vadez, V., 2010. Sources of tolerance to terminal drought in the chickpea (Cicer arietinum L.) mini core germplasm. Field Crops Res. 119, 322-330.

Krishnamurthy, L., Turner, N.C., Gaur, P.M., Upadhyaya, H.D., Varshney, R.K., Siddique, K.H.M., Vadez, V., 2011. Consistent variation across soil types in salinity resistance of a diverse range of chickpea (Cicer arietinum L.) genotypes. J.Agron. Crop Sci. http://dx.doi. org/10.1111/j.1439-037x.2010.00456.x.

Krivanek, A.F., Groote, G.D., Gunratna, N.S., Diallo, A.O., Friesen, D., 2007. Breeding and disseminating quality protein maize (QPM) for Africa. Afr. J. Biotechnol. 6, 312-324.

Kucharik, C.J., Serbin, S.P., 2008. Impact of recent climate change on Wisconsin corn and soybean yield trends. Environ. Res. Lett. 3.

Kumar, J., Abbo, S., 2001. Genetics of flowering time in chickpea and its bearing on productivity in semi-arid environments. Adv. Agron. 72, 107-138.

Kumar, L., Bandyopadhyay, R., 2012. Afla-ELISA: a simple and low-cost quantitative test for the estimation of aflatoxins. In: Kumar, L., Lopez, K. (Eds.), IITAR4D Review, 8, pp. 45-47, Special 45th Anniversary Edition.

Kumar, A., Bernier, J., Verulkar, S., Lafitte, H.R., Atlin, G.N., 2008. Breeding for drought tolerance: direct selection for yield, response to selection and use of drought-tolerant donors in upland and lowland-adapted populations. Field Crops Res. 107, 221-231.

Kumar, A.A., Reddy, B.V.S., Ramaiah, B., Reddy, P.S., Sahrawat, K.L., Upadhyaya, H.D., 2009. Genetic variability and plant character association of grain Fe and $\mathrm{Zn}$ in selected core collection accessions of sorghum germplasm and breeding lines. SAT eJ. 7.

Ladrera, R., Marino, D., Larrainzar, E., Gonzalez, E.M., Irrese-Igor, C., 2007. Reduced carbon availability to bacteroides and elevated ureides in nodules, but not in shoots, are involved in the nitrogen fixation response to early drought in soybean. Plant Physiol. $145,539-546$.

Ladygina, N., Henry, F., Kant, M.R., Koller, R., Reidinger, S., Rodriguez, A., Saj, S., Sonnemann, I., Witt, C., Wurst, S., 2010. Additive and interactive effects of functionally dissimilar soil organisms on a grassland community. Soil Biol. Biochem. 42, 2266-2275. 
Lake, J.A., Wade, R.N., 2009. Plant-pathogen interactions and elevated $\mathrm{CO}_{2}$ : morphological changes in favour of pathogens. J. Exp. Bot. 60, 3123-3131.

Lal, R., 2009. Soil degradation as a reason for inadequate human nutrition. Food Security $1,45-57$.

Lane, A., Jarvis, A., 2007. Changes in climate will modify the geography of crop suitability: agricultural biodiversity can help with adaptation. SAT eJ. 4.

La Porta, N., Capretti, P., Thomsen, I.M., Kasanen, R., Hietala, A.M., Von Weissenberg, K., 2008. Forest pathogens with higher damage potential due to climate change in Europe. Can. J. Plant Pathol. 30, 177-195.

Lee, G.J., Boerma, H.R., Villagarcia, M.R., Zhou, X., Carter Jr., T.E., Li, Z., Gibbs, M.O., 2004. A major QTL conditioning salt tolerance in S-100 soybean and descendent cultivars. Theor. Appl. Genet. 109, 1610-1619.

Lee, J.-D., Shannon, J.G., Choung, M.-G., 2011. Application of nondestructive measurement to improve soybean quality by near infrared reflectance spectroscopy. In: $\mathrm{Ng}, \mathrm{T}$.-B. (Ed.), Soybean - Applications and Technology, pp. 287-304. ISBN 978-953-307-207-4. InTech.

Lee, J.-D., Shannon, J.G., Vuong, T.D., Nguyen, H.T., 2009. Inheritance of salt tolerance in wild soybean (Glycine soja Sieb. and Zucc.) accession PI 483463. J. Hered. 100, 798-801.

Lewis, L., Onsongo, M., Njapau, H., Schurz-Rogers, H., Luber, G., Kieszak, S., Nyamongo, J., Backer, L., Dahiye, A.M., Misore, A., DeCock, K., Rubin, C., the Kenya Aflatoxicosis Investigation Group, 2005. Aflatoxin contamination of commercial maize products during an outbreak of acute aflatoxicosis in eastern and central Kenya. Environ. Health Perspect. 113, 1763-1767.

Lewis, J.E., Rowland, J., Nadeau, A., 1998. Estimating maize production in Kenya using NDVI: some statistical considerations. Int. J. Remote Sens. 19, 2609-2617.

Lenk, S., haerle, L., Pfündel, E.E., Longsdorf, G., Hagenbeek, D., Lichtenthaler, H.K., Van der Straeten, D., Buschmann, C., 2007. Multispectral fluorescence and reflectance imaging at the leaf level and its possible applications. J. Exp. Bot. 58, 807-814.

Lezar, S., Barros, E., 2010. Oligonucleotide microarray for the identification of potential mycotoxigenic fungi. BMC Microbiol. 10, 87.

Li, Y.B., Hu, Z.A., Wang, H.X., 2000. Further study on genotypic variation of salt tolerance to wild soybean (Glysine soja Sieb. and Zucc.). Soybean Genet. Newslett. http://www. soygenetics.prg./articles/sgn2000-016.htm.

Li, Z.K., Xu, J.L., 2007. Breeding for drought and salt tolerant rice (Oryza sativa L.): progress and perspectives. In: Jenks, M.A., Hasegawa, P.M., Jain, S.M. (Eds.), Advances in Molecular Breeding towards Drought and Salt Tolerant Crops, Springer,The Netherlands, pp. 531-564.

Lilley,J.M., Bolger, T.P., Peoples, M.B., Gifford, R.M., 2001. Nutritive value and the nitrogen dynamics of Trifolium subterraneum and Phalaris aquatica under warmer, high $\mathrm{CO}_{2}$ conditions. New Phytol. 150, 385-395.

Lippman, Z.B., Semel,Y., Zamir, D., 2007. An integrated view of quantitative trait variation using tomato interspecific introgression lines. Curr. Opin. Genet. Dev. 17, 545-552.

Liu, Y., Wu, F, 2010. Global burden of aflatoxin-induced hepatocellular carcinoma: a risk assessment. Environ. Health Perspect. 118, 818-824.

Lobell, D.B., Burke, M.B., Tebaldi, C., Mastrandrea, M.D., Falcon, W., Naylor, R., 2008. Prioritizing climate change adaptation need for food security in 2030. Science 319, 607-610.

Lobell, D.B., Field, C.B., 2007. Global scale climate - crop yield relationships and the impacts of recent warming. Environ. Res. Lett. 2. http://dx.doi.org/10.1088/17489326/2/1/014002.

Lobell, D.B., Schlenker,W., Costa-Roberts, J., 2011a. Climate trends and global crop production since 1980. Sci. Express. http://dx.doi.org/10.1126/science.1204531. 
Lobell, D.B., Bänziger, M., Magorokosho, C., Vivek, B., 2011b. Nonlinear heat effects on African maize as evidenced by historical yield trials. Nat. Clim. Change 1, 42-45.

Lobell, D.B., Sibley, A., Ortiz-Monasterio, J.I., 2012. Extreme heat effects on wheat senescence in India. Nat. Clim. Change. http://dx.doi.org/10.1038/nclimate1356.

Lobet, G., Pagès, L., Draye, X., 2011. A novel image-analysis toolbox enabling quantitative analysis of root system architecture. Plant Physiol. 157, 29-39.

Lorenz, A.J., Scott, M.P., Lamkey, K.R., 2007. Quantitative determination of phytate and inorganic phosphorous for maize breeding. Crop Sci. 47, 600-606.

Lott, J.N.A., Ockenden, I., Raboy,V., Batten, G.D., 2000. Phytic acid and phosphorus in crop seeds and fruits: a global estimate. Seed Sci. Res. 10, 11-33.

Luo,Y.Q., Hui, D.F., Zhang, D.Q., 2006. Elevated $\mathrm{CO}_{2}$ stimulates net accumulation of carbon and nitrogen in land ecosystems: a meta-analysis. Ecology 87, 53-63.

Luo, Q., Yu, B., Liu,Y., 2005. Differential sensitivity to chloride and sodium ions in seedlings of Glycine max and G. soja under NaCl stress. J. Plant Physiol. 162, 1003-1012.

Lussier,Y.A., Liu,Y., 2007. Computational approaches to phenotyping: high throughput phenomics. Proc. Am. Thorac. Soc. 4, 18-25.

Lyn, M.E., Abbas, H.K., Zablotowicz, R.M., Johnson, B.J., 2009. Delivery systems for biological control agents to manage aflatoxin contamination of pre-harvest maize. Food Addit. Contam. 26, 381-387.

Lynch, J.P., St. Clair, S.B., 2004. Mineral stress: the missing link in understanding how global climate change will affect plant in real world soils. Field Crops Res. 90, 101-115.

Mackay, A.D., Barber, S.A., 1985. Soil moisture effect on potassium uptake by corn. Agron. J. 77, 524-527.

Mackill, D.J., Ismail, A.M., Kumar,A., Gregorio, G.B., 2012. The role of stress-tolerant varieties for adapting to climate change. CURE Workshop Clim. Change, 4 May, 2010, Siem Reap, Cambodia.

Madgwick, J.W., West, J.S., White, R.P., Semenov, M.A., Townsend, J.A., Turner, J.A., Fitt, D.L., 2011. Impacts of climate change on wheat anthesis and fusarium ear blight in the UK. Euro. J. Plant Pathol. 130, 117-131.

Magan, N., Hope, R., Cairns, V., Aldred, D., 2003. Postharvest fungal ecology: impact of fungal growth and mycotoxin accumulation in stored grain. Euro. J. Plant Pathol. 107, $723-730$.

Magan, N., Medina, A., Aldred, D., 2011. Possible climate-change effects on mycotoxin contamination of food crops pre- and post-harvest. Plant Pathol. 60, 150-163.

Mahalakshmi, V., Subramanian, V., Bidinger, F.R., Jambunathan, R., 1985. Effect of water deficit on yield and protein content in pearl millet grains. J. Sci. Food Agric. 36, 1237-1242.

Mallikarjuna, N., Senthilvel, S., Hoisington, D., 2011. Development of new sources of tetraploid Arachis to broaden the genetic base of cultivated peanut (Arachis hypogaea L.). Genet. Resour. Crop Evol. 58, 889-907.

Manderscheid, R., Bender, J., Jager, H.J., Weigel, H.J., 1995. Effect of season long $\mathrm{CO}_{2}$ enrichment on cereals. II. Nutrient concentrations and grain quality. Agric. Ecosyst. Environ. 54, 175-185.

Margos, C.M., Busman, M., 2010. Rapid and advanced tools for mycotoxin analysis: a review. Food Addit.Contam. 27, 688-700.

Maxted, N., Kell, S.P., 2009. Establishment of a Global Network for the in Situ Conservation of Crop Wild Relatives: Status and Needs. FAO Commission on Genetic Resources for Food and Agriculture, Rome p. 266.

Maxted, N., Kell, S., Ford-Lloyd, B., Dulloo, E., Toledo, A., 2012. Toward the systematic conservation of global crop wild relative diversity. Crop Sci. 52, 774-785.

Maxted, N., Dulloo, E., Ford-Lloyd, B.V., Iriondo, J.M., Jarvis, A., 2008. Gap analysis: a tool for complementary genetic conservation assessment. Divers. Distrib. 14, 1018-1030. 
Matouš, K., Benediktyová, Z., Berger, S., Roitsch, T., Nedbal, L., 2006. Case study of combinatorial imaging: what protocol and what chlorophyll fluorescence image to use when visualizing infection of Arabidopsis thaliana by Pseudomonas syringae? Photosyn. Res. 90, 243-253.

McBeath, T.M., McLaughlin, M.J., Armstrong, R.J., Bell, M., Bolland, M.D.A., Conyers, M.K., Holloway, R.E., Mason, S.D., 2007. Predicting the response of wheat (Triticum aestivum L.) to liquid and granular phosphorus fertilizers in Australian soils. Aust. J. Soil Res. 45, 448-458.

McClean, P.E., Burridge, J., Beebe, S., Rao, I.M., Porch, T.G., 2011. Crop improvement in the era of climate change: an integrated, multi-disciplinary approach for common bean (Phaseolus vulgaris). Funct. Plant Biol. 38, 927-9337.

McLaughlin, J.F., Hellmann, J.J., Boggs, C.L., Ehrlih, P.R., 2002. Climate changes hasten population extinction. Proc. Natl. Acad. Sci. U S A 99, 6070-6074.

McMichael, A., Woodruff, R.E., Hales, S., 2006. Climate change and human health: present and future risks. Lancet 367, 859-869.

Meadows, M.E., 2003. Soil erosion in the Swartland, Western Cape Province, South Africa: implications of past and present policy and practice. Environ. Sci. Policy 6, 17-28.

Medina, A., Magan, N., 2012. Comparisons of three different $\mathrm{C}_{18}$ HPLC columns with different particle sizes for the optimization of aflatoxin analysis. J. Chromatogr. B 889-890, $138-143$.

Mehl, H.L., Cotty, P.J., 2010.Variation in competitive ability among isolates of $A$. flavus from different vegetative compatibility groups during infection. Phytopathology 100, 150-159.

Mehl, H.L., Cotty, P.J., 2011. Influence of the host contact sequence on the outcome of competition among Aspergillus flavus isolates during host tissue invasion. Appl. Environ. Microbiol. 77, 1691-1697.

Meilleur, A., Hodgkin, T., 2004. In situ conservation of crop wild relatives: status and trends. Biodivers. Conserv. 13, 663-684.

Melchinger,A.E., Schmidt, G., Geiger, H., 1986. Evaluation of near infrared reflectance spectroscopy for predicting grain and stover quality traits in maize. Plant Breed. 97, 20-29.

Menéndez, R., 2007. How are insects responding to global warming? Tijdschrift Voor Entomologie 150, 355-365.

Menkir, A., Brown, R.L., Bandyopadhyay, R., Chen, Z.-Y., 2006. A USA-Africa collaborative strategy for identifying, characterizing, and developing maize germplasm with resistance to aflatoxin contamination. Mycopathologia 162, 225-232.

Menzies, N.W., Kusumo, B., Moody, P.W., 2005. Assessment of P availability in heavily fertilized soils using the diffusive gradient in thin films (DGT) technique. Plant Soil 269, 1-9.

Mertz, E.T., Bates, L.S., Nelson, O.E., 1964. Mutant gene that changes protein composition and increases lysine content of maize endosperm. Science 145, 279-280.

Micklas, P.N., Kelly, J.D., Beebe, S.E., Blair, M.W., 2004. Common bean breeding for resistance against biotic and abiotic stresses: from classical to MAS breeding. Euphytica 147, 105-131.

Millan, T., Winter, P., Jüngling, R., Gil, J., Rubio, J., Cho, S., Cobos, M.J., Iruela, M., Rajesh, P.N., Takeoglu, M., Kahl, G., Muehlbauer, FJ., 2010. A consensus genetic map of chickpea (Cicer arietinum L.) based on 10 mapping populations. Euphytica 175, 175-179.

Miraglia, M., Marvin, H.J.P., Kleter, G.A., Battilani, P., Brera, C., Coni, E., Cubadda, F., Croci, L., De Santis, B., Dekkers, S., Filippi, L., Hutjes, R.W.A., Noordam, M.Y., Pisante, M., Piva, G., Prandini, A., Toti, L., van den Born, G.J., Vespermann, A., 2009. Climate change and food safety: an emerging issue with special focus on Europe. Food Chem. Toxicol. 47, 1009-1021.

Montes, J.M., Technow, F., Dhillon, B.S., Mauch, F., Melchinger,A.E., 2011. High throughput non-destructive biomass determination during early plant development in maize under field conditions. Field Crops Res. 121, 268-273. 
Mooney, S.J., Pridmore, T.P., Helliwell, J., Bennett, M.J., 2012. Developing X-ray computed tomography to non-invasively image 3-D root systems architecture in soil. Plant Soil $352,1-22$.

Moose, S.P., Dudley, J.W., Rocheford,T.R., 2004. Maize selection passes the century mark: a unique resource for 21st century genomics. Trends Plant Sci. 9, 358-364.

Moss, R.H., Edmonds, J.A., Hibbard, K.A., Manning, M.R., Rose, S.K., van Vuuren, D.P., Carter, T.R., Emori, S., Kainuma, M., Kram, T., Meehl, G.A., Mitchell, J.F.B., Nakicenovic, N., Riahi, K., Smith, S.J., Stouffer, R.J., Thomson, A.M., Weyant, J.P., Wilbanks, T.J., 2010. The next generation of scenarios for climate change research and assessment. Nature 463, 747-756.

Motley, T.J., Zerega, N., Cross, H., 2006. Darwin's Harvest. New Approach to the Origins, Evolution and Conservation of Crops. Columbia University Press, NY.

Muchero, W., Ehlers, J.D., Close, T.J., Roberts, P.A., 2009. Mapping QTL for drought stressinduced premature senescence and maturity in cowpea [Vigna unguiculata (L.) Walp.]. Theor. Appl. Genet. 118, 849-863.

Muchero, W., Ehlers, J.D., Roberts, P.A., 2008. Seedling stage drought-induced phenotypes and drought-responsive genes in diverse cowpea genotypes. Crop Sci. 48, 541-552.

Munns, R., James, R.A., Sirault, X.R.R., Furbank, R.T., Jones, H.G., 2010. New phenotyping methods for screening wheat and barley for beneficial response to water deficit. J. Exp. Bot. 61, 3499-3507.

Munns, R., Tester, M., 2008. Mechanisms of salinity tolerance. Ann. Rev. Plant Biol. 59, 651-681.

Muñoz-Perea, C.F., Terán, H., Allen, R.G., Wright, J.L., Westermann, D.T., Singh, S.P., 2006. Selection for drought resistance in dry bean landraces and cultivars. Crop Sci. 46, 2111-2120.

Nassar, N.M.A., Junior, O.P., Sousa, M.V., Ortiz, R., 2009. Improving carotenoids and aminoacids in cassava. Recent Patents Food Nutr. Agric. 1, 32-38.

Nayak, S.N., Zhu, H., Varghese, N., Choi, H.K., Datta, S., Horres, R., Jüngling, R., Singh, J., Kavi Kishor, P.B., Kahl, G., Winter, P., Cook, D.R., Varsheny, R.K., 2010. Integration of novel SSR and gene-based marker loci in the chickpea genetic map and establishment of new anchor points with Medicago truncatula genome. Theor. Appl. Genet. 120, 1415-1441.

Neelam, K., Rawat, N., Tiwari, V.K., Kumar, S., Chhuneja, P., Singh, K., Randhawa, G.S., Dhaliwal, H.S., 2010. Introgression of group 4 and 7 chromosomes of Ae. peregrina in wheat enhances grain iron and zinc density. Mol. Breed. http://dx.doi.org/10.1007/ s11032-010-9514-1.

Neeraja, C., Maghirang-Rodriguez, R., Pamplona, A.M., Heuer, S., Collard, B., Septiningsih, E., Vergara, G., Sanchez, D., Xu, K., Ismail, A., Mackill, D., 2007. A marker-assisted backcross approach for developing submergence-tolerance rice cultivars. Theor. Appl. Genet. 115, 767-776.

Nelson, O.E., Mertz, E.T., Bates, L.S., 1965. Second mutant gene affecting the amino acid pattern of maize endosperm proteins. Science 150, 1469-1470.

Nelson, M.R., Orum, T.V., Jaime-Garcia, R., Nadeem, A., 1999. Application of geographic information systems and geostatistics in plant disease epidemiology and management. Plant Dis. 83, 308-319.

Ni, J.J., Wu, P., Senadhira, D., Huang, N., 1998. Mapping QTLs for phosphorus deficiency tolerance in rice (Oryza sativa L.). Theor. Appl. Genet. 97, 1361-1369.

Nicotra, A.B., Davidson, A., 2010. Adaptive plasticity in water use traits. Funct. Plant Biol. 37, 117-127.

Nicotra, A.B., Taquín, O.K., Bonser, S.P., Davidson, A.M., Finnegan, E.J., Mathesius, U., Poot, P., Purugganan, M.D., Richards, C.L., Valladares, F., van Kleunen, M., 2010. Plant phenotypic plasticity in a changing climate. Trends Plant Sci. 15, 684-692. 
Nigam, S.N., Chandra, S., Sridevi, K.R., Bhukta, M., Reddy, A.G.S., Nageswara Rao, R.C., Wright, G.C., Reddy, P.V., Deshmukh, M.P., Mathur, R.K., Basu, M.S., Vasundhara, S., Varman, P.V., Nagda, A.K., 2005. Efficiency of physiological trait-based and empirical selection approaches for drought tolerance in groundnut. Ann. Appl. Biol. 146, 433-439.

Niklaus, P.A., Kröner, C., 2004. Synthesis of a six year study of calcareous grassland responses to in situ $\mathrm{CO}_{2}$ enrichment. Ecol. Monogr. 74, 491-511.

Norden, A.J., Gorbet, D.W., Knauft, D.A., Young, C.T., 1987.Variability in oil quality among peanut genotypes in the Florida breeding program. Peanut Sci. 14, 7-11.

Novotny, A.M., Schade, J.D., Hobbie, S.E., Kay, A.D., Kyle, M., Reich, P.B., Elser, J.J., 2007. Stoichiometric response of nitrogen-fixing and non-fixing dicots to manipulations of $\mathrm{CO}_{2}$, nitrogen, and diversity. Oceologia 151, 687-696.

Oatway, L., Vasanthan, T., Helm, J., 2001. Phytic acid. Food Rev. Int. 17, 417-431.

O’Brian, G.R., Georgianna, D.R., Wilkinson, J.R., Yu, J., Abbas, H.K., Bhatnagar, D., Nierman,W., Payne, G.A., 2007. The effect of elevated temperature on gene transcription and aflatoxin biosynthesis. Mycologia 99, 232-239.

Oktem, A., 2008. Effect of water shortage on yield, and protein and mineral compositions of drip-irrigated sweet corn in sustainable agricultural systems. Agric. Water Manage. 95, 1003-1010.

Olarte, R.A., Horn, B.W., Dorner, J.W., Monacell, J.T., Singh, R., Stones, E.A., Carbone, I., 2011. Effect of sexual recombination on population diversity in aflatoxin production by Aspergillus flavus and evidence for cryptic heterokaryosis. Mol. Ecol. http://dx.doi. org/10.1111/j.1365-294X.2011.05398.x.

Oren, R., Ellsworth, D.S., Johnsen, K.H., Phillips, N., Ewers, B.E., Maier, C., Schäfer, K.V.R., McCarthy, H., Hendrey, G., McNulty, S.G., Katul, G.G., 2001. Soil fertility limits carbon sequestration by forest ecosystems in a $\mathrm{CO}_{2}$-enriched atmosphere. Nature 411, 469-472.

Ortiz, R., 2011. Agrobiodiversity management for climate change. In: Lenné, J.M., Wood, D. (Eds.), Agrobiodiversity Management for Food Security, CAB International, Wallingford, Oxon, United Kingdom, pp. 189-211.

Ortiz, R., 2012. Climate Change and Agricultural Production. Technical Notes IDB-tn 383. Inter-American Development Bank, Washington D.C http://idbdocs.iadb.org/wsdocs/ getdocument.aspx?docnum $=36709950$.

Ortiz, R., Ban, T., Bandyopadhyay, R., Bänziger, M., Bergvinson, D., Hell, K., James, B., Jeffers, D., Kumar, P.L., Menkir, A., Murakami, J., Nigam, S.N., Upadhyaya, H.D., Waliyar, F, 2007. CGIAR research-for-development program on mycotoxins. In: Leslie, J.F,, Bandyopadhyay, R.,Visconti, A. (Eds.), Mycotoxins: Detection Methods, Management, Public Health and Agricultural Trade, CABI Publishing, Wallingford, United Kingdom, pp. 415-424.

Ortiz, R., Sayre, K.D., Govaerts, B., Gupta, R., Subbarao, G.V., Ban, T., Hodson, D., Dixon, J.M., Ortiz-Monasterio, J.I., Reynolds, M., 2008a. Climate change: can wheat beat the heat? Agric. Ecosyst. Environ. 126, 45-58.

Ortiz, R., Braun, H.J., Crossa, J., Crouch, J.H., Davenport, G., Dixon, J., Dreisigacker, S., Duveiller, E., He, Z., Huerta, J., Joshi, A.K., Kishii, M., Kosina, P., Manes, Y., Mezzalama, M., Morgounov, A., Murakami, J., Nicol, J., Ortiz-Ferrara, G., Ortiz-Monasterio, J.I., Payne, T.S., Peña, R.J., Reynolds, M.P., Sayre, K.D., Sharma, R.C., Singh, R.P., Wang, J., Warburton, M., Wu, H., Iwanaga, M., 2008b. Wheat genetic resources enhancement by the International Maize and Wheat Improvement Center (CIMMYT). Genet. Resour. Crop Evol. 55, 1095-1140.

Orum, T.V., Bigelow, D.M., Cotty, P.J., Nelson, M.R., 1999. Using predictions based on geostatistics to monitor trends in Aspergillus flavus strain composition. Phytopathology 89, 761-769. 
Osborne, S.L., Schepers, J.S., Francis, D.D., Schlemmer, M.R., 2002. Use of spectral radiance to estimate in-season biomass and grain yield in nitrogen and water stressed corn. Crop Sci. 42, 165-171.

O'Shaughnessy, S.A., Evett, S.R., Colaizzi, P.D., Howell, T.A., 2011. Using radiation thermography and thermometry to evaluate crop water stress in soybean and cotton. Agric. Water Manag. 98, 1523-1535.

Owensby, C.E., Cochran, R.C., Auen, L.M., 1996. Effects of Elevated Carbon dioxide on Forage Quality for Ruminants. Academic Press, Inc, pp. 363-371.

Ozturk, A., Aydin, F., 2004. Effect of water stress at various growth stages on some quality characteristics of winter wheat. J. Agron. Crop Sci. 190, 93-99.

Paez, A.V., Helm, J.L., Zuber, M.S., 1969. Lysine content of opaque 2 maize kernels having different phenotypes. Crop Sci. 9, 251-252.

Pagani, M., Zachos, J.C., Freeman, K.H., Tipple, B., Bohaty, S., 2005. Marked decline in atmospheric carbon dioxide concentrations during the Paleogene. Science 309, 600-6003.

Pal, M., Karthikeyapandian, V., Jain, V., Srivastava, A.C., Raj, A., Sengupta, U.K., 2004. Biomass production and nutritional levels of berseem (Trifolium alexandrium) grown under elevated $\mathrm{CO}_{2}$. Agric. Ecosyst. Environ. 101, 31-38.

Palm, C.A., Smukler, S.M., Sullivan, C.C., Mutuo, P.K., Nyadzi, G.I., Walsh, M.G., 2010. Identifying potential synergies and trade-offs for meeting food security and climate change objectives in sub-Saharan Africa. Proc. Natl. Acad. Sci. U S A 107, 19661-19666.

Paltridge, N.G., Palmer, L.J., Milhan, P.J., Guild, G.E., Stangoulis, J.C.R., 2012. Energy-dispersive X-ray fluorescence analysis of zinc and iron concentration in rice and pearl millet grain. Plant Soil. http://dx.doi.org/10.1007/s11104-011-1104-4.

Pang, J., Zhu, J.-G., Xie, Z.-B., Liu, G., Zhang,Y.-L., Chen, G.-P., Zheng, Q., Cheng, L., 2005. A new explanation of the $\mathrm{N}$ concentration decrease in tissues of rice (Oryza sativa L.) exposed to elevated atmospheric $\mathrm{pCO}_{2}$. Environ. Exp. Bot. 57, 98-105.

Paoletti, M.G., Foissner, W., Coleman, D. (Eds.), 1994. Soil Biota, Nutrient Cycling, and Farming Systems, Lewis Publishers, Boca Raton, p. 314.

Papa, 1986. Heterokaryon incompatibility in Aspergillus flavus. Mycologia 78, 98-101.

Parker, M.B., Gascho, G.J., Gains, T.P., 1983. Chloride toxicity of soybean grown on Atlantic Coast flatwoods soils. Agron. J. 75, 439-443.

Parmesan, C., Yohe, G., 2003. A globally coherent fingerprint of climate change impacts across natural systems. Nature 421,37-42.

Pascale, M.N., 2009. Detection methods for mycotoxins in cereal grains and cereal products. Proc. Nat. Sci. Matica Srpska Novi Sad No 117, 15-25.

Paterson, R.R.M., Lima, N., 2010. How will climate change affect mycotoxins in food? Food Res. Int. 43, 1902-1914.

Paterson, R.R.M., Lima, N., 2011. Further mycotoxin effects from climate change. Food Res. Int. 44, 2555-2566.

Patil, A.G., Oak, M.D., Taware, S.P., Tamhankar, S.A., Rao,V.S., 2010. Nondestructive estimation of fatty acid composition in soybean [Glycine max (L.) Merrill] seeds using nearinfrared transmittance spectroscopy. Food Chem. 120, 1210-1217.

Patz, J.A., Kovats, R.S., 2002. Hot spots in climate change and human health: present and future risks. Lancet 368, 859-869.

Patz, J.A., Olson, S.H., 2006. Climate change and health: global to local influences on disease risk. Ann. Trop. Med. Parasitol. 100, 535-549.

Peleg, Z., Reguera, M., Walia, H., Blumwald, E., 2011. Cytokinin mediated source-sink modifications improve drought tolerance and increases grain yield in rice under water stress. Plant Biotechnol. J. 9, 747-758.

Peleg, Z., Saranga, Y., Yazici, A., Fahima, T., Ozturk, L., Cakmak, I., 2008. Grain zinc, iron and protein concentrations and zinc-efficiency in wild emmer wheat under contrasting irrigated regimes. Plant Soil 306, 57-67. 
Pellegrineschi, A., Reynolds, M., Pacheco, M., Brito, R.M., Almeraya, R., Yamaguchi-Shinozaki, K., Hoisington, D., 2004. Stressed induced expression in wheat of the Arabidopsis thaliana DREB1A gene delays water stress symptoms under greenhouse conditions. Genome 47, 493-500.

Peñuelas, J., 1998.Visible and near-infrared reflectance techniques for diagnosing plant physiological status. Trends Plant Sci. 3, 151-156.

Pfeiffer, W.H., McClafferty, B., 2007. HarvestPlus: breeding crops for better nutrition. Crop Sci. 47 (S3), S88-S105.

Phang, T., Shao, G., Lam, H., 2008. Salt tolerance in soybean. J. Integr. Plant Biol. 50, 1196-1212.

Pit, J.I., Hocking, A.D., 2006. Mycotoxin in Australia: biocontrol of aflatoxin in peanut. Mycopathologia 162, 233-243.

Porteaus, F., Hill, J., Ball, A.S., Pinter, P.J., Kimball, B.A., Wall, G.W., Adamsen, FJ., Hunsaker, D.J., LaMorte, R.L., Leavitt, S.W., Thompson, T.L., Matthias, A.D., Brooks, T.J., Morris, C.F., 2009. Effect of free air carbon dioxide enrichment (FACE) on the chemical composition and nutritive value of wheat grain and straw. Anim. Feed Sci. Technol. 149, 322-332.

Prasad, B., Carver, B.F., Stone, M.L., Baber, M.A., Raun, W.R., Klatt, A.R., 2007a. Genetic analysis of indirect selection for winter wheat grain yield using spectral reflectance indices. Crop Sci. 47, 1416-1425.

Prasad, B., Carver, B.F., Stone, M.L., Baber, M.A., Raun, W.R., Klatt, A.R., 2007b. Potential use of spectral reflectance indices as a selection tool for grain yield in winter wheat under Great Plains conditions. Crop Sci. 47, 1426-1440.

Prasad, P.V.V., Staggenborg, S.A., Ristic, Z., 2008. Impacts of drought and/or heat stress on physiological, developmental, growth and yield processes of crop plants. Response of crops to limited water: Understanding and modeling water stress effects on plant growth processes Adv. Agric. Syst. Model. Ser. 1, 301-355.

Prichard, S.G., 2011. Soil organisms and global climate change. Plant Pathol. 60, 82-99.

Probst, C., Bandyopadhyay, R., Price, L.E., Cotty, P.J., 2011. Identification of atoxigenic Aspergillus flavus isolates to reduce aflatoxin contamination of maize in Kenya. Plant Dis. 95, 212-218.

Pugliese, M., Gullino, M.L., Garibaldi,A., 2010. Effects of elevated $\mathrm{CO}_{2}$ and temperature on interactions of grapevine and powdery mildew: first results under phytotron conditions. J. Plant Dis. Prot. 117, 9-14.

Qin, H., Gu, Q., Zhang, J., Sun, L., Kuppu, S., Zhang,Y., Burow, M., Payton, P., Blumwald, E., Zhang, H., 2011. Regulated expression of Isopentenyltransferase gene (IPT) in peanut significantly improves drought tolerance and increases yield under field conditions. Plant Cell Physiol. 52, 1904-1914.

Ramirez-Villegas, J., Lau, C., Koehler, A.-K., Singer, J., Jarvis, A., Arnell, N., Osborne, T., Hooker, J., 2011. Climate Analogues: Finding Tomorrow's Agriculture Today. Working Paper No. 12. CGIAR (Consultative Group on International Agricultural Research) Research Program on climate Change Agriculture and Food Security (CCAFS), Cali, Colombia Available online at www.ccafs.cgiar.org.

Ramirez-Vallejo, P., Kelly, J.D., 1998. Traits related to drought resistance in common bean. Euphytica 99, 127-138.

Rana, J.C., Sharma, S.K., 2009. Plant genetic resource management under emerging climate change. Indian J. Genet. 69, 1-17.

Rascher, U., Blossfeld, S., Fiorani, F., Jahnke, S., Jansen, M., Kuhn, A.J., Matsubara, S., Martin, L.L.A., Merchant, A., Metzner, R., Müuller-Linow, M., Nagel, K.A., Pieruschka, R., Pinto, F., Schreiber, C.M., Temperton, V.M., Thorpe, M.R., van Dusschoten, D., van Volkenburgh, E., Windt, C.W., Schurr, U., 2011. Non-invasive approaches for phenotyping of enhanced performance traits in bean. Funct. Plant Biol. 38, 968-983. 
Rastija, M., Kovacevic, V., Rastija, D., Simic, D., 2010. Manganese and zinc concentrations in maize genotypes grown on soils differing in acidity. Acta Agronomica Hungarica 58, 385-393.

Ravi, K.,Vadez,V., Isobe, S., Mir, R.R., Guo, Y., Nigam, S.N., Gowda, M.V.C., Radhakrishnan, T., Bertioli, D.J., Knapp, S.J., Varshney, R.K., 2011. Identification of several small main- effect QTLs and a large number of epistatic QTLs for drought tolerance related traits in groundnut (Arachis hypogaea L.). Theor. Appl. Genet. 122, 1119-1132.

Reich, P.B., 2009. Elevated $\mathrm{CO}_{2}$ reduces losses of plant diversity caused by nitrogen deposition. Science 326, 1399-1402.

Reich, P.B., Hungate, B.A., Luo, Y., 2006a. Carbon nitrogen interactions in terrestrial ecosystems in response to rising atmospheric carbon dioxide. Annu. Rev. Ecol. Evol. Syst. 37, 611-636.

Reich, P.B., Hobbie, S.E., Lee, T., Ellsworth, D.S., West, J.B., Tilman, D., Knops, J.M.H., Naeem, S., Trost, J., 2006b. Nitrogen limitation constrains sustainability of ecosystem response to $\mathrm{CO}_{2}$. Nature 440, 922-925.

Ren, S., Weeda, S., Li, H., Whitehead, B., Guo, Y., Atalay, A., Parry, J., 2012. Salt tolerance in soybean WF-7 is partially regulated by ABA and ROS signaling and involves withholding toxic $\mathrm{Cl}^{-}$ions from aerial tissues. Plant Cell. Rep. http://dx.doi.org/10.1007/ s00299-012-1268-2.

Rennie, B.D., Tanner, J.W., 1989. Fatty acid composition of oil from soybean seeds grown at extreme temperatures. J. Am. Oil Chem. Soc. 66, 1622-1624.

Reuzeau, C., Pen, J., Frankard, V., de Wolf, J., Peerbolte, R., Broekaert, W., van Camp, W., 2005. TraitMill ${ }^{\mathrm{TM}}$ : a discovery engine for identifying yield-enhancement genes in cereals. Mol. Plant Breed. 5, 753-759.

Reynolds, M., 2010. Climate Change and Crop Production. CAB International,Wallingford, UK.

Reynolds, M.P., Rajaram, S., Sayre, K.D., 1999. Physiological and genetic changes of irrigated wheat in the post-green revolution period and approaches for meeting projected global demand. Crop Sci. 39, 1611-1621.

Ribeiro, J.M.M., Cavaglieri, L.R., Fraga, M.E., Direito, G.M., Dalcero, A.M., Rosa, C.A.R., 2006. Influence of water activity, temperature and time on mycotoxins production on barley rootlets. Lett. Appl. Microbiol. 42, 179-184.

Rivero, R.M., Kojima, M., Gepstein, A., Sakakibara, H., Mittler, R., Gepstein, S., Blumwald, E., 2007. Delayed leaf senescence induces extreme drought tolerance in a flowering plant. Proc. Natl. Acad. Sci. U S A 104, 19631-19636.

Rivero, R.M., Shulaev, V., Blumwald, E., 2009. Cytokinin-dependent photorespiration and the protection of photosynthesis during water deficit. Plant Physiol. 150, 1380-1393.

Rivero, M., Walia, H., Blumwald, E., 2010. Cytokinin-dependent protection of photosynthetic protein complexes during water deficit. Plant Cell Physiol. 51, 1929-1941.

Rodriguez-Amaya, D.B., Kimura, M., 2004. HarvestPlus Handbook for Carotenoid Analysis. HarvestPlus Technical Monograph 2. International Food Policy Research Institute (IFPRI) and International Center for Tropical Agriculture (CIAT), Washington, DC and Cali.

Ronald, P., 2011. Plant genetics, sustainable agriculture and global food security. Genetics $188,11-20$.

Roos, J., Hopkins, R., Kvarnheden, A., Dixelius, C., 2011. The impact of global warming on plant diseases and insect vectors in Sweden. Eur. J. Plant Pathol. 129, 9-19.

Rosales, A., Galicia, L., Oviedo, E., Islas, C., Palacios-Rojas, N., 2011. Near-infrared reflectance spectroscopy (NIRS) for protein, tryptophane, and lysine evaluation in quality protein maize (QPM) breeding programs. J. Agric. Food Chem. 59, 10781-10786.

Roudier, P., Sultan, B., Quirion, P., Berg, A., 2011. The impact of future climate change on west African crop Yields: what does the recent literature say? Glob. Environ. Change 21, 1073-1083. 
Royo, C., Aparicio, N.,Villegas, D., Casadesus, J., Monneveux, P., Araus, J.L., 2003. Usefulness of spectral reflectance indices as durum wheat yield predictors under contrasting Mediterranean conditions. Int. J. Remote Sens. 24, 4403-4419.

Rudolphi, S., Becker, H.C., Schierholt, A., von Witzke-Ehbrecht, S., 2012. Improved estimation of oil, linoleic and oleic acid and seed hull fractions in safflower by NIRS. J. Am. Oil Chem. Soc. 89, 363-369.

Rudorff, B.F.T., Batista, G.T., 1990. Spectral response of wheat and its relationship to agronomic variables in the tropical regions. Remote Sens. Environ. 31, 53-63.

Russell, R., Ptterson, M., Lima, N., 2010. How will climate change affect mycotoxins in food? Food Res. Int. 43, 1902-1914.

Sadok, W., Naudin, P., Boussuge, B., Muller, B., Belcker, C., Tardieu, F., 2007. Leaf growth rate per unit thermal time follows QTL-dependent daily patterns in hundreds of maize lines under naturally fluctuating conditions. Plant Cell Environ. 30, 135-146.

Sadok, W., Sinclair, T.R., 2009. Genetic variability of transpiration response to vapor pressure deficit among soybean cultivars. Crop Sci. 49, 955-956.

Sahrawat, K.L., 2009. The role of tolerant genotypes and plant nutrients in reducing acid-soil infertility in upland rice ecosystem: an appraisal. Arch. Agron. Soil Sci. 55, 597-607.

Sahrawat, K.L., Abekoe, M.K., Diatta, S., 2001. Application of inorganic phosphorus fertilizer. In:Tian, G., Ishida, F., Keatinge, D. (Eds.), Sustaining Soil Fertility in West Africa, Soil Science Society of America Special Publication no. 58, Soil Sci. Soc. Am. And Am. Soc. Agron, Madison, WI, USA, pp. 225-246.

Sahrawat, K.L., Kumar, G.R., Rao, J.K., 2002. Evaluation of triacid and dry ashing procedures for determining potassium, calcium, magnesium, iron, zinc, manganese and copper in plant materials. Commn Soil Sci. Pl. Anal. 33, 95-102.

Sahrawat, K.L., Wani, S.P., 2013. Soil testing as a tool for on-farm fertility management: experience from the semi-arid zone of India. Commun. Soil Sci. Pl.Anal. 44, 1011-1032.

Sahrawat, K.L., Wani, S.P., Subba Rao, A., Pardhasaradhi, G., 2011. Management of emerging multi-nutrient deficiencies: a prerequisite for sustainable enhancement of rainfed agricultural productivity. In: Wani, S.P., Rockstrom, J., Sahrawat, K.L. (Eds.), Integrated Watershed Management in Rainfed Agriculture, CRC Press/Balkema, 2300 AK Leiden, The Netherlands, pp. 281-313.

Saint Pierre, C.S., Crossa, J.L., Bonnett, D., Yamaguchi-Shinozaki, K., Reynolds, M.P., 2012. Phenotyping transgenic wheat for drought resistance. J. Exp. Bot. 63, 1799-1808.

Saito, K., Sokei, Y., Wopereis, M.C.S., 2012. Enhancing rice productivity in West Africa through genetic enhancement. Crop Sci. 52, 484-493.

Salas Fernandez, M.G., Hamblin, M.T., Li, L., Rooney, W.L., Tuinstra, M.L., Kresovich, S., 2008. Quantitative trait loci analysis of endosperm color and carotenoids content in sorghum grain. Crop Sci. 48, 1732-1743.

Salas Fernandez, M.G., Kapran, I., Souley, S., Abdou, M., Maiga, I.H., Acharya, C.B., Hamblin, M.T., Kresovich, S., 2009. Collection and characterization of yellow endosperm sorghums from West Africa for biofortification. Genet. Resour. Crop Evol. 56, 991-1000.

Sambatti, J.B.M., Caylor, K.K., 2007. When is breeding for drought tolerant optimal if drought is random? New Phytol. 175, 70-80.

Sanchez, P.A., 2002. Soil fertility and hunger in Africa. Science 295, 2019-2020.

Sanchis, V., Magan, N., 2004. Environmental conditions affecting mycotoxins. In: Magan, N., Olsen, M. (Eds.), Mycotoxins in Food: Detection and Control, Woodhead Publishing Ltd, Cambridge, UK, pp. 174-189.

Sarkar, R.K., Bhattacharjee, B., 2012. Rice genotypes with SUB1 QTL differ in submergence tolerance, elongation ability during submergence and regeneration growth at re-emergence. Rice 5, 7. 
Sarkar, R.K., Panda, D., Reddy, J.N., Patnaik, S.S.C., Mackil, D.J., Ismail, A.M., 2009. Performance of submergence tolerant rice (Oryza sativa) genotypes carrying the Sub1quantitative trait locus under stressed and non-stressed natural field conditions. Indian J. Agric. Sci. 79, 876-883.

Savary, S., Nelson, A., Sparks, A.H., Willocquet, L., Duveiller, E., Mahuku, G., Forbes, G., Garrett, K.A., Hodson, D., Padgham, J., Pande, S., Sharma, M., Yuen, J., Djurle, A., 2011. International agricultural research tackling the effects of global and climate changes on plant diseases in the developing world. Plant Dis. 95, 1204-1216.

Schimel, J., Balser, T.C., Wallenstein, M., 2007. Microbial stress-response physiology and its implications for ecosystem function. Ecology 88, 1386-1394.

Schlenker, W., Lobell, D.W., 2010. Robust negative impacts of climate change on African agriculture. Environ. Res. Lett. 5.

Schlenker, W., Roberts, M.J., 2009. Nonlinear temperature effects indicate severe damages to US crop yields under climate change. Proc. Natl. Acad. Sci. U S A 106, 15594-15598.

Schmidt-Heydt, M., Abdel-Hadi, A., Magan, N., Geisen, R., 2009. Complex regulation of the aflatoxin biosynthesis gene cluster of Aspergillus flavus in relation to various combinations of water activity and temperature. Int. J. Food Microbiol. 135, 231-237.

Schmidt-Heydt, M., Geisen, R., 2007. A microarray for monitoring the production of mycotoxins in food. Int. J. Food Microbiol. 117, 131-140.

Schmidt-Heydt, M., Magan, N., Geisen, R., 2008. Stress induction of mycotoxin biosynthesis genes by abiotic factors. FEMS Microbiol. Lett. 284, 142-149.

Schmidt-Heydt, M., Parra, R., Geisen, R., Magan, N., 2011. Modelling the relationship between environmental factors, transcriptional genes and deoxynivalenol mycotoxin production by strains of two Fusarium species. J. R. Soc. Interface 8, 117-126.

Schmidt-Heydt, M., Rüfer, C.E., Abdel-Hadi, A., Magan, N., Geisen, R., 2010. The production of aflatoxin $\mathrm{B}_{1}$ or $\mathrm{G}_{1}$ by Aspergillus parasiticus at various combinations of temperature and water activity is related to the ratio of aflS to aflR expression. Mycotox. Res. 26, 241-246.

Schneider, K.A., Brothers, M.E., Kelly, J.F., 1997. Marker-assisted selection to improve drought resistance in common bean. Crop Sci. 37, 51-60.

Seneweera, S.P., Conroy, J.P., 1997. Growth, grain yield and quality of rice (Oryza sativa L.) in response to elevated $\mathrm{CO}_{2}$ and phosphorus nutrition. Soil Sci. Plant Nutr. 43, 1131-1136.

Septiningsih, E.M., Pamplona, A.M., Sanchez, D.L., Neeraja, C.N., Vergara, G.V., Heuer, S., Ismail, A.M., Mackill, D.J., 2009. Development of submergence-tolerant rice cultivars: the Sub1 locus and beyond. Ann. Bot. 103, 151-160.

Shahryari, R., Valizadeh, M., Mollasadeghi, V., 2011. Evaluation of irrigation levels and its impact on quality and quantity performance of wheat. Adv. Environ. Biol. 5, 528-534.

Shamseddin, A.M., Adeeb, A.M., 2012. Using remotely sensed and ancillary data to predict spatial variability of rainfed crop yield. Int. J. Remote Sens. 33, 3798-3815.

Shanthi Prabha,V., Sreekanth, N.P., Babu, P.K., Thomas, A.P., 2011.The trilemma of Soil Carbon Degradation, Climate Change and Food Insecurity. pp. 107-112 Disaster, Risk and Vulnerability Conference 2011, School of Environmental Sciences, Mahatma Gandhi University, India - The Applied Geoinformatics for Society and Environment, Germany.

Sharma, H.C., Mukuru, S.Z., Manyasa, E., Were, J., 1999. Breakdown of resistance to sorghum midge, Stenodiploisis sorghicola. Euphytica 109, 131-140.

Shaw, M.W., 2009. Preparing for changes in plant disease due to climate change. Plant Protect. Sci. 45, S3-S10.

Shaw, M.W., Osborne, T.M., 2011. Geographic distribution of plant pathogens in response to climate change. Plant Pathol. 60, 31-43.

Sheridan, J.A., Bickford, D., 2011. Shrinking body size as an ecological response to climate change. Nat. Clim. Change. http://dx.doi.org/10.1038/nclimate1259. 
Shewry, P.R., Halford, N.G., 2002. Cereal seed storage proteins: structure, properties and role in grain utilization. J. Exp. Bot. 53, 947-958.

Simpson, C.E., Starr, J.L., 2001. Registration of COAN peanut. Crop Sci. 41, 918.

Simpson, C.E., Starr, J.L., Church, G.T., Burow, M.D., Paterson, A.H., 2003a. Registration of 'Nema TAM' peanut. Crop Sci. 43, 1561.

Simpson, C.E., Baring, M.R., Schubert, A.M., Melouk, H.A., Lopez, Y., Kirby, J.S., 2003 b. Registration of 'OLin' peanut. Crop Sci. 43, 1880.

Simpson, C.E., Starr, J.L., Nelson, S.C., Woodard, K.E., Smith, O.D., 1993. Registration of TxAG-6 and TxAG-7 peanut germplasm. Crop Sci. 33, 1418.

Sinclair, T.R., 1986. Water and nitrogen limitations in soybean grain production. I. Model development. Field Crops Res. 15, 125-141.

Sinclair, T.R., 1992. Mineral nutrition and plant growth response to climate change. J. Exp. Bot. 43, 1141-1146.

Sinclair, A.H., Edwards, A.C., 2008. Micronutrient deficiency problems in agricultural crops in Europe. In:Alloway, B.J. (Ed.), Micronutrient Deficiencies in Global Crop Production, 9, pp. 225-244.

Sinclair, T.R., Hammer, G.L., van Oosterom, E.J., 2005. Potential yield and water use efficiency benefits in sorghum from limited maximum transpiration rate. Funct. Plant Biol. 32, 945-952.

Sinclair,T.R., Messina, C.D., Beatty, A., Samples, M., 2010. Assessment across United States of the benefits of altered soybean drought traits. Agron. J. 102, 475-482.

Sinclair, T.R., Pinter Jr., P.J., Kimball, B.A., Adamsen, FJ., LaMorte, R.L., Wall, G.W., Hunsaker, D.J., Adam, N., Brooks, T.J., Garcia, R.L., Thompson, T., Leavitt, S., Matthias, A., 2000. Leaf nitrogen concentration of wheat subjected to elevated $\mathrm{CO}_{2}$ and either water or $\mathrm{N}$ deficits. Agric. Ecosyst. Environ. 79, 53-60.

Sinclair, T.R., Zwieniecki, M.A., Holbrook, N.M., 2008. Low leaf hydraulic conductance associated with drought tolerance in soybean. Physiol. Plant 132, 446-451.

Singh, S.P., 1995. Selection for water-stress tolerance in interracial populations of common bean. Crop Sci. 35, 118-124.

Singh, S., Singh, G., Singh, P., Singh, N., 2008a. Effect of water stress at different stages of grain development on the characteristics of starch and protein of different wheat varieties. Food Chem. 108, 130-139.

Singh, R.P., Hodson, D.P., Huerta-Espino, J., Jin, Y., Njau, P., Waynera, R., Herrera-Foessel, S.A., Ward, R.W., 2008b. Will stem rust destroy the world's wheat crop? Adv. Agron. 98, 271-309.

Singh, S., Mackill, D.J., Ismail, A.M., 2009. Responses of SUB1rice introgression lines to submergence in the field: yield and grain quality. Field Crops Res. 113, 12-23.

Sirault, X.R.R., Jones, R.A., Furbank, R.T., 2009. A new screening method for osmotic component of salinity tolerance in cereals using infrared thermography. Funct. Plant Biol. 36, 970-977.

Soriano, I.R., Riley, T.T., Potter, M.J., Bowers, W.S., 2004. Phytoecdysteroids: a novel defense against plant-parasitic nematodes. J. Chem. Ecol. 30, 1885-1899.

Sparks, D.L., 2003. Environmental Soil Chemistry, second ed. Academic Press, Amsterdam, the Netherlands.

Spielbauer, G., Armstrong, P., Baier, J.W., Allen, W.B., Richardson, K., Shen, B., Settles, A.M., 2009. High-throughput near-infrared reflectance spectroscopy for predicting quantitative and qualitative composition phenotypes of individual maize kernels. Cereal Chem. $86,556-564$.

St. Clair, S.B., Lynch, J.P., 2010.The opening of pandora's box: climate change impacts on soil fertility and crop nutrition in developing countries. Plant Soil 335, 101-115.

Stein, A.J., 2010. Global impacts of human mineral nutrition. Plant Soil 335, 133-154. 
Steven, J.B., Diane, F.K., 2002. Warm temperature and drought during seed maturation increases free $\alpha$-tocopherol in the seed of soybean. J. Agric. Food Chem. 50, 6058-6063.

Stevens, C.J., Dise, N.B., Mountford, J.O., Gowing, D.J., 2004. Impact of nitrogen deposition on the species richness of grasslands. Science 303, 1876-1879.

Sturrok, R.N., Frankel, S.J., Brown, A.V., Hennon, P.E., Kliejunas, T., Lewsi, K.J., Worrall, J.J., Woods, A.J., 2011. Climate change and forest diseases. Plant Pathol. 60, 133-149.

Suding, K.N., Collins, S.L., Gough, L., Clark, C., Cleland, E.E., Gross, K.L., Milchunas, D.G., Pennings, S., 2005. Functional- and abundance-based mechanisms explain diversity loss due to N fertilization. Proc. Natl. Acad. Sci. U S A 102, 4387-4392.

Sun, Y., Yin, J., Cao, H., Li, C., Kang, L., Ge, F., 2011. Elevated $\mathrm{CO}_{2}$ influences nematodeinduced defense responses of tomato genotypes differing in the JA pathway. PLoS One 6 e19751.

Sundaram, J., Kandala, C.V., Govindrajan, K.N., Subbiah, J., 2012. Sensing of moisture content of in-shell peanuts by NIR reflectance spectroscopy. J. Sensor Technol. 2, 1-7.

Sutherst, R.W., Constable, F., Finlay, K.J., Harrington, R., Luck, J., Zaluck, M.P., 2011. Adapting to crop pest and pathogen risks under a changing climate. WIREs Clim. Change 2, 220-237.

SWPGRFA, 2009. Draft second report on the state of world plant genetic resources for food and agriculture (CGRFA-12/09/Inf.rRev.1). Twelth Regular Session, 19-23 Oct 2009, Rome, Italy.

Sylvain, Z.A., Wall, D.H., 2011. Linking soil biodiversity and vegetation: implications for a changing planet. Am. J. Bot. 98, 517-527.

Tackenberg, O., 2007. A new method for non-destructive measurement of biomass, growth rates, vertical biomass distribution and dry matter content based on digital image analysis. Ann. Bot. 99, 777-783.

Tajuddin, T., Watanabe, S., Masuda, R., Harada, K., Kawano, S., 2002. Application of nearinfrared spectroscopy to the estimation of protein and lipid contents in single seeds of soybean recombinant inbred for quantitative trait loci analysis. J. Near Infrared Spectrosc. 10, 315-325.

Takeda, S., Matsuoka, M., 2008. Genetic approaches to crop improvement: responding to environmental and population changes. Nat. Rev. Genet. http://dx.doi.org/10.1038/nrg2342.

Tallada, J., Palacios-Rojas, N., Armstrong, P., 2009. Prediction of maize seed attributes using a rapid single kernel near infrared instrument. J. Cereal Sci. 50, 381-387.

Tandy, S., Mundus, J., Yngvesson, J., de Bang, T.C., Lombi, E., Schjoerring, J.K., Husted, S., 2011. The use of DGT for prediction of plant available copper, zinc and phosphorus in agricultural soils. Plant Soil 346, 167-180.

Tang, J.L., Zhang, B., Gao, C., Zepp, H., 2008. Hydrological pathway and source area of nutrient losses identified by a multi-scale monitoring in an agricultural catchment. Catena 72, 374-385.

Taub, D.R., Miller, B., Allen, H., 2008. Effects of elevated $\mathrm{CO}_{2}$ on the protein concentration of food crops: a meta-analysis. Glob. Change Biol. 14, 565-575.

Teow, C.C., Truong, V.-D., McFeeters, R.F., Thompson, R.L., Pecota, K.V., Yencho, G.C., 2007. Antioxidant activities, phenolic and $\beta$-carotene contents of sweet potato genotypes with varying flesh colours. Food Chem. 103, 829-838.

Terán, H., Singh, S.P., 2002. Comparison of sources and lines selected for drought resistance in common bean. Crop Sci. 42, 64-70.

Tester, M., Langridge, P., 2010. Breeding technologies to increase crop production in a changing world. Science 327, 818-822.

Tiwari,V.K., Rawat, N., Neelam, K., Kumar, S., Randhawa, G.S., Dhaliwal, H.S., 2010. Substitutions of $2 \mathrm{~S}$ and $7 \mathrm{U}$ chromosomes of Aegilops kotschy in wheat enhance grain iron and zinc concentrations. Theor. Appl. Genet. 121, 259-269. 
Thomas, J.M.G., Boote, K.J., Allen, L.H., Gallo-Meagher, M., Davis, J.M., 2003. Elevated temperature and carbon dioxide effects on soybean seed composition and transcript abundance. Crop Sci. 43, 1548-1557.

Thomas, C.D., Cameron, A., Green, R.E., Bakkenes, M., Beaumont, L.J., Collingham, Y.C., Erasmus, B.F.N., de Siqueira, M.F., Grainger, A., Hannah, L., Hughes, L., Huntley, B., van Jaarsveld, A.S., Midgley, G.F., Miles, L., Ortega-Huerta, M.A., Peterson, A.T., Phillips, O.L., Williams, S.E., 2004. Extinction risk from climate change. Nature 427, 145-148.

Thomas, J.M.G., Prasad, P.V.V., Boote, K.J., Allen Jr., L.H., 2009. Seed composition, seedling emergence and early seedling vigour of red kidney bean seed produced at elevated temperature and carbon dioxide. J. Agron. Crop Sci. 195, 148-156.

Thomson, M.J., Ismail, A.M., McCouch, S.R., Mackill, D.J., 2010. Marker-assisted breeding. In: Pareek, A., Sopory, S.K., Bohnert, H.J. (Eds.), Abiotic Stress Adaptation in Plants: Physiological, Molecular and Genetic Foundation, Springer, New York (USA), pp. 451469.

Thornton, P.K., Jones, P.G., Ericksen, P.J., Challinor, A.J., 2011. Agriculture and food systems in sub-Saharan Africa in a 4 C+ world. Phil. Trans. R. Soc. A. Math. Phys. Eng. Sci. 369, $117-136$.

Tirado, M.C., Clarke, R., Jaykus, L.A., McQuatters-Gollop, A., Frank, J.M., 2010. Climate change and food safety: a review. Food Res. Int. 43, 1745-1765.

Trachsel, S., Kaeppler, S.M., Brown, K.M., Lynch, J.P., 2010. Shovelomics: high throughput phenotyping of maize (Zea mays L.) root architecture in the field. Plant Soil. http://dx.doi.org/10.1007/s11104-010-0623-8.

Upadhyaya, H.D., 2005. Variability for drought resistance related traits in the mini core collection of peanut. Crop Sci. 45, 1432-1440.

Upadhyaya, H.D., 2008. Crop germplasm and wild relatives: a source of noble variation for crop improvement. Korean J. Crop Sci. 53, 12-17.

Upadhyaya, H.D., Bramel, P.J., Ortiz, R., Singh, S., 2002. Developing a mini core of peanut for utilization of genetic resources. Crop Sci. 42, 2150-2156.

Upadhyaya, H.D., Ortiz, R., 2001. A mini core collection for capturing diversity and promoting utilization of chickpea genetic resources in crop improvement. Theor. Appl. Genet. 102, 1292-1298.

Upadhyaya, H.D., Pundir, R.P.S., Dwivedi, S.L., Gowda, C.L.L., 2009. Mini Core Collections for Efficient Utilization of Plant Genetic Resources in Crops Improvement Programs. Information Bull. no. 78. ICRISAT, Patancheru, India pp. 52. ISBN 978-92-9066-51-9.

Upadhyaya, H.D., Ramesh, S., Sharma, S., Singh, S.K., Varshney, S.K., Sharma, N.D.R.K., Ravishankar, C.R., Narsimhudu, Y., Reddy, V.G., Sahrawat, K.L., Dhanalakshmi, T.N., Mgonja, M.A., Parzies, H.K., Gowda, C.L.L., Singh, S., 2011a. Genetic diversity for grain nutrients contents in a core collection of finger millet (Eleusine coracana (L.) Gaertn.) germplasm. Field Crops Res. http://dx.doi.org/10.1016/j/fcr.2010.11.017.

Upadhyaya, H.D., Ravishankar, C.R., Narasimhudu,Y., Sarma, N.D.R.K., Singh, S.K.,Varshney, S.K., Reddy, V.G., Singh, S., Parzies, H.K., Dwivedi, S.L., Nadaf, H.L., Sahrawat, K.L., Gowda, C.L.L., 2011b. Identification of trait-specific germplasm and developing a mini core collection for efficient use of foxtail millet genetic resources in crop improvement. Field Crops Res. 124, 457-467.

Upadhyaya, H.D., Mukri, G., Nadaf, H.L., Singh, S., 2011c. Variability and stability analysis for nutritional traits in the mini core collection of peanut. Crop Sci. 52, 168-178.

Upadhyaya, H.D., Dronavalli, N., Gowda, C.L.L., Singh, S., 2011d. Identification and evaluation of chickpea germplasm for tolerance to heat stress. Crop Sci. 51, 2079-2094.

Upadhyaya, H.D., Dwivedi, S.L., Ambrose, M., Ellis, N., Berger, J., Smýkal, P., Debouck, D., Duc, G., Dumet, D., Flavell, A., Sharma, S.K., Mallikarjuna, N., Gowda, C.L.L., 2011e. Legume genetic resources: management, diversity assessment, and utilization in crop improvement. Euphytica. http://dx.doi.org/10.1007/s10681-011-0449-3. 
Uprety, D.C., 2007. Effect of rising atmospheric $\mathrm{CO}_{2}$ on the productivity of crop plants, pp. 1-26 in National Fellow Report (ICAR).

Uprety, D.C., Bisht, B.S., Dwivedi, N., Saxena, D.C., Mohan, R., Raj, A., Paswan, G., Mitra, A.P., Garg, S.C., Tiwari, M.K., Maini, H.K., Singh, D., 2007. Comparison between open top chamber (OTC) and free air $\mathrm{CO}_{2}$ Enrichment (FACE) technologies to study the response of rice cultivars to elevated $\mathrm{CO}_{2}$. Physiol. Mol. Biol. Plants 13, 259-266.

Uprety, D.C., Dwivedi, N., Raj, A., Jaiswal, S., Paswan, G., Jain,V., Maini, H.K., 2009. Study on the response of diploid, tetraploid and hexaploid species of wheat to the elevated $\mathrm{CO}_{2}$. Physiol. Plant Mol. Biol. Plants 15, 161-168.

U. S. Environmental Protection Agency, 2003. Biopesticide registration action document Aspergillus flavus AF36. www.epa.gov/oppbppd1/biopesticides/ingredients/tech_ docs/brad_006456.pdf.

U. S. Environmental Protection Agency, 2004. Biopesticide registration action document Aspergillus flavus (NRRL 21882). www.epa.gov/oppbppd1/biopesticides/ingredients/ tech_docs/brad_006456.pdf.

Vadez, V., Krishnamurthy, L., Serraj, R., Gaur, P.M., Upadhyaya, H.D., Hoisington, D.A., Varshney, R.K., Turner, N.C., Siddique, K.H.M., 2007a. Large variation in salinity tolerance in chickpea is explained by differences in sensitivity at the reproductive stage. Field Crops Res. 104, 123-129.

Vadez, V., Rao, S., Sharma, K.K., Bhatnagar-Mathur, P., 2007b. DREB1 Aallows for more water uptake by a large modification in the root/shoot ratio under water deficit. Int. Arachis Newslett. 27, 27-31.

Vadez,V., Rao, S., Kholova, J., Krishnamurthy, L., Kashiwagi, J., Ratnakumar, P., Sharma, K.K., Bhatnagar-Mathur, P., Basu, P.S., 2008. Root research for legume tolerance to drought: Quo vadis? J. Food Legumes 21, 77-85.

van der Heijden, M.G.A., Bardgett, R.D., van Straalen, N.M., 2008. The unseen majority: soil microbes as drivers of plant diversity and productivity in terrestrial ecosystems. Ecol. Lett. 11, 296-310.

Vardon, C., McLaughlin, C., Nardinelli, C., 2003. Potential economic costs of mycotoxins in the United States. Council for Agricultural Science and Technology Task Force Report No. 139.

Varsheny, R.K., Close, T.J., Singh, N.K., Hoisington, D.A., Cook, D.R., 2009a. Orphan legume crops enter the genomics era!. Curr. Opin. Plant Biol. 12, 202-210.

Varsheny, R.K., Hiremath, P.J., Lekha, P.T., Kashiwagi, J., Balaji, J., Deokar, A.A., Vadez, V., Xiao, Y., Srinivasan, R., Gaur, P.M., Siddique, K.H.M., Town, C.D., Hoisington, D.A., 2009b. A comprehensive resource of drought- and salinity-responsive ESTs for gene discovery and marker development in chickpea (Cicer arietinum L.). BMC Genomics 10,523 .

Varshney, R.K., Hoisington, D.A., Upadhyaya, H.D., Gaur, P.M., Nigam, S.N., Saxena, K., Vadez,V., Sethy, N.K., Bhatia, S., Aruna, A., Gowda, M.V.C., Singh, N.K., 2007. Molecular genetics and breeding of grain legume crops for the semi-arid tropics. In:Varsheny, R.K., Tuberosa, R. (Eds.), Genomics Assisted Crop Improvement, Genomics Applications in Crops, vol. II. Springer, The Netherlands, pp. 207-242.

Vasal, S.K., 1999. Improving human nutrition through agriculture: the role of international agricultural research. I. Quality protein maize story. A workshop hosted by IRRI and organized by IFPRI, Oct 5-7, 1999.

Vasyukova, N.I., Zinov'eva, S.V., Udalova, Z.V., Panina, Y.S., Ozeretskovskaya, O.L., Sonin, M.D., 2003. The role of salicylic acid in systemic resistance of tomato to nematodes. Dokl. Biol. Sci. 391, 419-421.

Venuprasad, R., Lafitte, R., Atlin, G.N., 2007. Response to direct selection for grain yield under drought stress in rice. Crop Sci. 47, 285-293. 
Vermeulen, S., Zougmore, R., Wollenberg, E., Thornton, P., Nelson, G., Kristjanson, P., Kinyangi, J., Jarvis, A., Hansen, J., Challinor, A., Campbell, B., Aggrawal, P., 2011. Climate change, agriculture and food security: a global partnership to link research and action for low-income agricultural producers and consumers. Curr.Opin. Environ. Sustainability 4, 1-6.

Vigouroux,Y., Mariac, C., de Mita, S., Pham, J.-L., Gérard, B., Kapran, J., Sagnard, F., Deu, M., Chantereau, J., Ali, A., Ndjeunga, J., Luong, V., Thuillet, A.-C., Saïdou, A.-A., Bezançon, G., 2011. Selection for early flowering crop associated with climatic variations in the Sahel. PLoS One 6e19563.

Waliyar, F., Reddy, S.V., Lava-Kumar, P., 2009. Review of immunological methods for the quantification of aflatoxins in peanut and other foods. Peanut Sci. 36, 54-59.

Wall, D.H., Bardgett, R.D., Kelly, E.F., 2010. Biodiversity in the dark. Nat. Geosci. 3, 297-298.

Walter, A., Scharr, H., Gilmer, F, Zierer, R., Nagel, K.A., Ernst, M., Wiese, A., Virnich, O., Christ, M.M., Uhlig, B., Juenger, S., Schurr, U., 2007. Dynamics of seedling growth acclimation towards altered light conditions can be quantified via GROWSCREEN: a setup and procedure designed for rapid optical phenotyping of different plant species. New Phytol. 174, 447-455.

Wang, X., Cai, J., Liu, F., Jin, M., Yu, H., Jiang, D., Wolleweber, B., Dai, T., Cao, W., 2012. Pre- anthesis high temperature acclimation alleviates the negative effects of post-anthesis heat stress on stem stored carbohydrates remobilization and grain starch accumulation in wheat. J. Cereal Sci. http://dx.doi.org/10.1016/j/jcs.2012.01.004.

Wardle, D.A., 2002. Communities and Ecosystems: Linking the Aboveground and Belowground Components. Princeton University Press, Princeton N J, USA.

Weber, W.S., Melchinger, A.E., Magorokosh, C., Makumbi, D., Bänzinger, M., Atlin, G.N., 2012. Efficiency of managed-stress screening of elite maize hybrids under drought and low nitrogen for yield under rainfed conditions in southern Africa. Crop Sci. 52, 1011-1020.

Weegels, P.L., Hammer, R.J., Schofield, J.D., 1996. Critical review: functional properties of wheat glutenin. J. Cereal Sci. 23, 1-18.

Welch, R.M., 2002. The impact of mineral nutrients in food crops on global human health. Plant Soil 247, 83-90.

West, J.S., Holdgate, S., Townsend, J.A., Edwards, S.G., Jennings, P., Fitt, B.D.L., 2012. Impacts of changing climate and agronomic factors on fusarium ear blight of wheat in the UK. Fungal Ecol. 5, 53-61.

Wheal, M.S., Fowles, T.O., Palmer, L.T., 2011. A cost-effective acid digestion method using closed polypropylene tubes for inductively coupled plasma optical emission spectrometry (ICP-OES) analysis of plant essential elements. Anal. Methods 3, 2854-2863.

Wiegand, C.L., Richardson, A.J., Escobar, D.E., Gerbermann, A.H., 1991.Vegetation indices in crop assessments. Remote Sens. Environ. 35, 105-119.

Wieser, H., 2007. Chemistry of gluten proteins. Food Microbiol. 24, 115-119.

Wieser, H., Manderscheid, R., Erbs, M., Weigel, H.-J., 2008. Effects of elevated atmospheric $\mathrm{CO}_{2}$ concentrations on the quantitative protein composition of wheat grain. J. Agric. Food Chem. 56, 6531-6535.

Williams, J.H., Grubb, J.A., Davis, J.W., Wang, J.S., Jolly, P.E., Ankrah, N.A., Ellis, W.O., Afriyie-Gyawu, E., Jonson, N.M., Robinson, A.G., Phillips, T.D., 2010. HIV and hepatocellular and esophageal carcinomas related to consumption of mycotoxin-prone foods in sub-Saharan Africa. Am. J. Clin. Nutr. 92, 154-160.

Williams, J.H., Phillips, T.D., Jolly, P.E., Stiles, J.K., Jolly, C.M., Aggarawal, D., 2004. Human aflatoxicosis in developing countries: a review of toxicology, exposure, potential health consequences, and interventions. Am. J. Clin. Nutr. 80, 1106-1122.

Wissuwa, M., Ae, N., 2001a. Genotypic variation for tolerance to phosphorus deficiency in rice and the potential for exploitation in rice improvement. Plant Breed. 120, 43-48. 
Wissuwa, M.,Ae, N., 2001b. Further characterization of two QTLs that increase phosphorus uptake of rice (Oryza sativa L.) under phosphorus deficiency. Plant Soil 237, 275-286.

Wissuwa, M.,Wegner, J., Ae, N.,Yano, M., 2002. Substitution mapping of Pup 1: a major QTL increasing phosphorus uptake of rice from a phosphorus deficient soil. Theor. Appl. Genet. 105, 890-897.

Wissuwa, M.,Yano, M.,Ae, N., 1998. Mapping of QTLs for phosphorus deficiency tolerance in rice (Oryza sativa L.). Theor. Appl. Genet. 97, 777-783.

Winterhalter, L., Mistele, B., Jampatong, S., Schmidhalter, U., 2011. High throughput phenotyping of canopy water mass and canopy temperature in well-watered and drought stressed tropical maize hybrids in the vegetative stage. Euro. J. Agron. 35, 22-32.

Wittkop, B., Snowdon, R.J., Friedt, W., 2012. New NIRS calibrations for fiber fractions reveal broad genetic variation in Brassica napus seed quality. J. Agric. Food Chem. 60, $2248-2256$.

Wolf, R.B., Cavins, J.F., Kleman, R., Black, L.T., 1982. Effect of temperature on soybean seed constituents: oil, protein, moisture, fatty acids, amino acids and sugars. J. Am. Oil Chem. Soc. 59, 230-232.

Wright, G.C., Rachupati, N.C., Chauhan, Y.S., Robson, A., 2005. Increasing Productivity and Quality of Peanuts Using Novel Crop Modeling and Remote Sensing Technologies. pp. 14-17. International Peanut Conference on Prospects and Emerging Opportunities for Peanut Quality and Utilization Technology, Kasetsart University, Bangkok, Thailand 9-12 January 2005.

Wrigley, C., 2006. Global warming and wheat quality. Cereals Food World 51, 34-36.

WSFS (World Summit on Food Security), Declaration of the world summit on food security. http://www.fao.org/fileadmin/templates/wsfs/summit2009.

Wu, F., Narrod, C., Tiongco, M., Liu, Y., The health economics of aflatoxin: global burden of disease. IFPRI working paper on aflacontrol - Improving lives in Africa (http://www. ifpri.org/sites/default/files/publications/aflacontrol_wp04.pdf2011a.

Wu, F., Bhatnagar, D., Bui-Klimke, T., Carbone, I., Hellmich, R., Munkvold, G., Paul, P., Payne, G., Takle, E., 2011b. Climate change impacts on mycotoxin risks in US maize. World Mycotoxin J. 4, 79-93.

Wu, D.-X., Wang, G.-X., Bai,Y.-F., Liao, J.-X., 2004. Effects of elevated $\mathrm{CO}_{2}$ concentration on growth, water use, yield and grain quality of wheat under two soil water levels. Agric. Ecosyst. Environ. 104, 493-507.

Xiao, J., Grandillo, S., Ahn, S.N., McCouch, S.R., Tanksley, S.D., Li, J., Yuan, L., 1996. Genes from wild rice improve yield. Nature 384, 223-224.

Xie, W., Nevo, E., 2008. Wild emmer: genetic resources, gene mapping and potential for wheat improvement. Euphytica 164, 603-614.

Xu, Z., Chang, R., Qiu, L., Sun, J., Li, X., 1999. Evaluation of Soybean Germplasm in China. pp. 156-165. Proc. World Soybean Conf.VI. Chicago, 4-7 August 1999, Superior Printing, Champagne.

Xu, K., Mackill, D.J., 1996. A major locus for submergence tolerance mapped on rice chromosome 9. Mol. Breed. 2, 219-224.

Xu, K., Xia, X., Fukao, T., Canlas, P., Maghirang-Rodriguez, R., Heuer, S., Ismail, A.M., Bailey-Serres, J., Ronald, P.C., Mackill, D.J., 2006. Sub1A is a ethylene response factorlike gene that confers submergence tolerance to rice. Nature 442, 705-708.

Xu, Z., Yanzhuo, L., Qingni, K., Nongrong, H., Yi, C., Daijan, P., 1998. Growth, grain yield and kernel quality of high-yield rice variety Te-San-Ai 2 growing in a simulated $\mathrm{CO}_{2}-$ enriched habitat. Chin. J. Appl. Environ. Biol. 4, 238-242.

Yadav, R.S., Hash, C.T., Bidinger, F.R., Cavan, G.P., Howart, C.J., 2002. Quantitative trait loci associated with traits determining grain and stover yield in pearl millet under terminal drought-stress conditions. Theor. Appl. Genet. 104, 67-83. 
Yadav, R.S., Hash, C.T., Bidinger, F.R., Devos, K.M., Howart, C.J., 2004. Genomic regions associated with grain yield and aspects of post-flowering drought tolerance in pearl millet across stress environments and testers background. Euphytica 136, 265-277.

Yan, J., Kandianis, C.B., Harjes, C.E., Bai, L., Kim, E.-H., Yang, X., Skinner, D.J., Fu, Z., Mitchell, S., Li, Q., Fernandez, M.G.S., Zaharieva, M., Babu, R., Fu, Y., Palacios, N., Li, J., DellaPenna, D., Brutnell, T., Buckler, E.S., Warburton, M.L., Rocheford, T., 2010. Rare genetic variation at Zea mays $c r t R B I$ increases $\beta$-carotene in maize grain. Nat. Genet. $42,322-327$.

Yáñez-López, R., Torres-Pacheco, I., Guevara-González, R.G., Hernández-Zul, M.I., Quijano-Carranza, J.A., Rico-García, E., 2012. The effect of climate change on plant diseases. Afr. J. Biotechnol. 11, 2417-2428.

Yang, Z., Sinclair, T.R., Zhu, M., Messina, C.D., Cooper, M., Hammer, G.L., 2012.Temperature effect on transpiration response of maize plants to vapor pressure deficit. Environ. Exp. Bot. 78, 157-162.

Yang, X.B., Sun, P., Hu, B.H., 1998. Decadal change of plant diseases as affected by climate in Chinese agroecosystems. Int. Plant Pathol. Congress, Edinburg, U.K. 3:4.2.1 (Abstr.).

Yin, Y., Lou, T., Michailides, T.J., Ma, Z., 2009. Molecular characterization of toxigenic and atoxigenic Aspergillus flavus isolates, collected from peanut fields in China.J. Appl. Microbiol. 107, 1857-1865.

Yu, J., Chang, P.-K., Ehrlich, K.C., Cary, J.W., Bhatnagar, D., Cleveland, T.E., Payne, G.A., Linz, J.E., Woloshuk, C.P., Bennett, J.W., 2004. Clustered pathway genes in aflatoxin biosynthesis. Appl. Environ. Microbiol. 70, 1253-1262.

Yuan, L.P., 1993. Advantages and constraints to use of hybrid rice varieties. In: Wilson, K.J. (Ed.), Proc. Int. Workshop on Apomixis in Rice, The Rockfeller Foundation, New York and Chinese National Center for Biotechnology Development, Beijing, China, pp. 1-4.

Zahir, Z.A., Arshad, M., Frankenberger Jr., W.T., 2004. Plant growth promoting rhizobacteria: applications and perspective in agriculture. Adv. Agron. 81, 97-168.

Zaman-Allah, M., Jenkinson, D.M., Vadez, V., 2011a. A conservative pattern of water use, rather than deep or profuse rooting, is critical for the terminal drought tolerance of chickpeas. J. Exp. Bot. http://dx.doi.org/10.1093/jxb/err139.

Zaman-Allah, M., Jenkinson, D.M., Vadez, V., 2011b. Chickpea genotypes contrasting for seed yield under terminal drought stress in the field differ for traits related to control of water use. Funct. Plant Biol. 38, 270-281.

Zamir, D., 2008. Plant breeders go back to nature. Nat. Genet. 40, 5-6.

Zavala, J.A., Casteel, C.K., DeLucia, E.H., Berenbaum, M.R., 2008. Anthropogenic increase in carbon dioxide compromises plant defense against invasive insects. Proc. Natl. Acad. Sci. U S A 105, 5129-5133.

Zavaleta, E.S., Shaw, M.R., Chiariello, N.R., Mooney, H.A., 2003. Additive effects of simulated climate changes, elevated $\mathrm{CO}_{2}$, and nitrogen deposition on grassland diversity. Proc. Natl. Acad. Sci. U S A 100, 7650-7654.

Zhang, H., Davison,W., Gadi, R., Kobayashi,T., 1998. In situ measurement of dissolved phosphorus in natural waters using DGT. Anal. Chim. Acta 370, 29-38.

Zhang, T., Wang, Z., Yin, Y., Cai, R., Yan, S., Li, W., 2010. Starch content and granule size distribution in grains of wheat in relation to post-anthesis water deficits. J. Agron. Crop Sci. 196, 1-8.

Zhang, H., Zhao, FJ., Sun, B., Davison, W., McGrath, S.P., 2001. A new methods to measure effective soil solution concentration predicts copper availability to plants. Environ. Sci. Technol. 35, 2602-2607.

Zhao, C.-X., He, M.-R., Wang, Z.-L., Wang, Y.-F., Lin, Q., 2009. Effect of different water availability at post-anthesis stage on grain nutrition and quality in strong-gluten winter wheat. C. R. Biol. 332, 759-764. 
Zheng, P., Allen, W.B., Roesler, K., Williams, M.E., Zhang, S., Li, J., Glassman, K., Ranch, J., Nubel, D., Solawetz, W., Bhattramakki, D., Llaca, V., Deschamps, S., Zhong, G.-Y., Tarczynski, M.C., Shen, B., 2008. A phenylalanine in DGAT is a key determinant of oil content and composition in maize. Nat. Genet. 40, 367-372.

Zhili, H., Xu, M., Deng, Y., Kang, S., Kellog, L., Wu, L., Van Nostrand, J.D., Hobbie, S.E., Reich, P.B., Zhou, J., 2010. Metagenomic analysis reveals a marked divergence in the structure of belowground microbial communities at elevated $\mathrm{CO}_{2}$. Ecol. Lett. 13, 564575 .

Ziska, L.H., Epstein, P.R., Schlesinger, W.H., 2009. Rising $\mathrm{CO}_{2}$, climate change, and public health: exploring the links to plant biology. Environ. Health Perspect. 117, 155-158.

Ziska, L.H., Morris, C.F., Goins, E.W., 2004. Quantitative and qualitative evaluation of selected wheat varieties released since 1903 to increasing atmospheric carbon dioxide: can yield sensitivity to carbon dioxide be a factor in wheat performance? Glob. Change Biol. 10, 1810-1819.

Ziska, L.H., Namuco, O., Moya, T., Qilang, J., 1997. Growth and yield response of fieldgrown tropical rice to increasing carbon dioxide and air temperature. Agron. J. 89, 45-53.

Ziska, L.H., Palowsky, R., Reed, D.R., 2007. A quantitative and qualitative assessment of mung bean (Vigna mungo (L.) Wilczek) seed in response to elevated atmospheric carbon dioxide: potential changes in fatty acid composition. J. Sci. Food Nutr. 87, 920-923.

Zougmore, R., Mando, A., Stroosnijder, L., 2009. Soil nutrient and sediment loss as affected by erosion barriers and nutrient source in semi-arid Burkina Faso. Arid Land Res. Manag. 23, 85-101. 